\title{
THE CHIRAL PHASE TRANSITION IN QCD: CRITICAL PHENOMENA AND LONG WAVELENGTH PION OSCILLATIONS
}

\author{
KRISHNA RAJAGOPAL \\ Lyman Laboratory of Physics, Harvard University, \\ Cambridge, MA, USA. 02138 \\ E-mail: rajagopal@huhepl.harvard.edu
}

\begin{abstract}
In QCD with two flavours of massless quarks, the chiral phase transition is plausibly in the same universality class as the classical four component Heisenberg magnet. Therefore, renormalization group techniques developed in the study of phase transitions can be applied to calculate the critical exponents which characterize the scaling behaviour of universal quantities near the critical point. As a consequence of this observation, a quantitative description of the universal physics of the chiral phase transition in circumstances where the plasma is close to thermal equilibrium as it passes through the critical temperature can be obtained. This approach to the QCD phase transition has implications both for lattice gauge theory and for heavy ion collisions. Future lattice simulations with longer correlation lengths will provide quantitative measurements of the various exponents and the equation of state for the order parameter as a function of temperature and quark masses. Present lattice simulations already allow many qualitative tests. In a heavy ion collision, the consequence of a long correlation length would be large fluctuations in the number ratio of neutral to charged pions. Unfortunately, we will see that because of the explicit chiral symmetry breaking introduced by the masses of the up and down quarks, no equilibrium correlation length gets long enough for this phenomenon to occur. In the third chapter of this article, I discuss attempts to model the dynamics of the chiral order parameter in a far from equilibrium QCD phase transition by considering quenching in the $\mathrm{O}(4)$ linear sigma model. I argue, and present numerical evidence, that in the period immediately following the quench long wavelength modes of the pion field are amplified. This could have dramatic phenomenological consequences in heavy ion collisions. I review various recent theoretical advances - attempts to include expansion and relax the quench assumption; attempts to include quantum mechanical effects. Long wavelength pion oscillations have now been seen in a number of simulations in which different assumptions are made. However, all of the theoretical treatments involve idealizations and assumptions, and should only be regarded as qualitative guides to what can happen. It is up to experimentalists to determine whether or not such phenomena occur; detection in a heavy ion collision would imply an out of equilibrium chiral transition.
\end{abstract}

To appear in Quark-Gluon Plasma 2, edited by R. Hwa, World Scientific, 1995.

April, 1995.

HUTP-95-A013 


\section{Introduction}

Shortly after the discovery that QCD is asymptotically free ${ }^{\mathbb{A}}$ and that therefore quarks are almost noninteracting at short distances, Collins and Perrye noted that this means that QCD at high temperature or high density is qualitatively different than at low temperature and density. At high temperatures $\left(T \gg \Lambda_{\mathrm{QCD}}\right)$ or high baryon number density $\left(n \gg \Lambda_{\mathrm{QCD}}^{-3}\right)$ QCD describes a world of weakly interacting quarks and gluons very different from the hadronic world in which we live. This raises the possibility of phase transitions as the temperature or density is increased. While the subject of phase transitions as a function of increasing density has interesting applications in the physics of neutron stars and heavy ion collisions, in this article I will limit myself to discussing the physics of a QCD plasma with zero baryon number density. There are at least two qualitative differences between such a plasma at $T \gg \Lambda_{\mathrm{QCD}}$ and at $T \sim 0$. First, as Collins and Perry discussed, at low temperatures one has a plasma of quarks and gluons confined in mesons and baryons, while at high temperatures the quarks and gluons are deconfined. Second, at low temperatures a $q \bar{q}$ condensate spontaneously breaks chiral symmetry while at high temperatures the interactions among the quarks and anti-quarks are weak, no such condensate exists, and chiral symmetry is manifest. Thus, in studying the phase structure of QCD as a function of increasing temperature, there are two possible phase transitions to consider.

Studying the QCD phase transition(s) has a certain intrinsic appeal. The question involved is simple enough that it can be stated in a sentence: What happens if you take a chunk of any substance around you and heat it up until it is one hundred thousand times hotter than the centre of the sun? While the question is simple, if one tries to answer it experimentally by colliding heavy ions at ultra-relativistic energies, the phenomena seem at first glance complicated and intractable. And yet, using techniques from different areas of theoretical physics - quantum field theory, particle physics, nuclear physics, lattice gauge theory, condensed matter physics, and cosmology - much progress is possible. Quantitative predictions for processes in which a plasma cools through the QCD phase transition in thermal equilibrium can be extracted, and striking phenomena with qualitative signatures may occur if the plasma is far from equilibrium as it cools through the transition.

In addition to having intrinsic appeal, the QCD phase transition is of interest from several different points of view. First, there can be no doubt that it occurred throughout the universe about a microsecond after the big bang. Second, it is reasonable to hope that in a heavy ion collision of sufficiently high energy, a small region of the high temperature phase is created which then cools through the phase transition. Third, lattice gauge theory is well suited to calculating the equilibrium properties of QCD 
at high temperatures. From all these perspectives, it is important to learn as much as can be learned analytically about the phase transition, relying as much as possible only on fundamental symmetries and universality arguments and as little as possible on specific assumptions and models. In Chapter 2, following original work done with Wilczek, 3 we will see that it is possible to use this approach to learn much about the equilibrium QCD phase transition. In studying heavy ion collisions, however, it is worth considering the possibility that the transition process may be far from thermal equilibrium. Without thermal equilibrium, it is much harder to be quantitative both because the phenomena are more varied and because there is no notion of universality. We will nevertheless find that the distinctive qualitative signature of the QCD phase transition in heavy ion collisions discussed in Chapter 3 - the amplification of long wavelength oscillations of the pion field - can only occur if the plasma is far from thermal equilibrium as it experiences the QCD phase transition.

The physics of QCD at high temperatures is an enormous subject with many facets, which I will not attempt to review here. The reader interested in entering the voluminous literature might consider starting with the review by Gross, Pisarski, and Yaffe, 0 the review by McLerran, 6 the book by Shuryak, 7 the previous collection of articles edited by Hwa, recent reviews by Müller, $\frac{1}{6}$ recent conference proceedings in the Quark Matter series, 10,11,12.13 or other articles in this volume.

The remainder of this introduction has two goals. First, a brief discussion of the confinement/deconfinement phase transition seems in order, since the bulk of this article is concerned with the chiral phase transition. Second, I will give a very brief introduction to some of the physics relevant for a discussion of the QCD phase transition in heavy ion collisons, in order to set the stage for the third chapter.

Let us recall the arguments 14 which lead to the conclusion that the confinement/deconfinement phase transition is first order in pure $S U(3)$ gauge theory and is smoothed into a crossover when light quarks are present. In studying $S U(N)$ gauge theory in thermal equilibrium at a temperature $T$, the partition function is given by a Euclidian path integral

$$
Z=\int_{\mathrm{PBC}} \mathcal{D} A_{\mu} \exp \left(-S_{E}\right)
$$

over a slab of spacetime which is infinite in the three spatial directions and is $\beta \equiv 1 / T$ thick in the $t$ direction. $A_{\mu} \equiv A_{\mu}^{a} \lambda^{a}$ where the $\lambda^{a}$ are representation matrices for the fundamental representation of $S U(N)$. The path integral is over gauge field configurations which satisfy periodic boundary conditions $A_{\mu}(\vec{x}, 0)=A_{\mu}(\vec{x}, \beta)$. The Euclidian action is given by

$$
S_{E}=\frac{1}{4 g^{2}} \int_{0}^{\beta} \mathrm{d} t \int \mathrm{d}^{3} \vec{x} \operatorname{tr} F_{\mu \nu} F_{\mu \nu} .
$$

The expectation value of operators is given by

$$
\left\langle\mathcal{O}\left(A_{\mu}\right)\right\rangle=Z^{-1} \int_{\mathrm{PBC}} \mathcal{D} A_{\mu} \exp \left(-S_{E}\right) \mathcal{O}\left(A_{\mu}\right) .
$$


We will be particularly interested in the Wilson loop operator

$$
L(\vec{x})=\operatorname{tr} \Omega(\vec{x}) ; \quad \Omega(\vec{x})=P \exp \left[i \int_{0}^{\beta} \mathrm{d} t A_{0}(\vec{x}, t)\right]
$$

for a loop at fixed $\vec{x}$ which loops around the periodic time direction once. $P$ denotes path ordering. This operator, conventionally called the Polyakov loop, is of interest because the free energy of a single static quark is

$$
\beta\left(F_{q}-F_{0}\right)=-\log \langle L(\vec{x})\rangle
$$

where $F_{0}=-T \log Z$ is the free energy of the vacuum. If $\langle L\rangle=0$, inserting a quark into the ensemble costs an infinite free energy. This implies that the flux cannot be screened - the state of the $A_{\mu}$ fields is changed all the way out to spatial infinity and costs an infinite free energy. Therefore, $\langle L\rangle=0$ indicates that the theory is in the confining phase. If $\langle L\rangle \neq 0$, then $F_{q}$ is finite and the theory is in a deconfined phase characterized by Debye screening.

Under a gauge transformation

$$
A_{\mu} \rightarrow U A_{\mu} U^{-1}+i U \partial_{\mu} U^{-1}, \quad U(\vec{x}, t) \in S U(N)
$$

$L$ transforms as

$$
L(\vec{x}) \rightarrow \operatorname{tr} U(\vec{x}, 0) \Omega(\vec{x}) U(\vec{x}, \beta),
$$

so $L$ is invariant when $U$ is periodic. Local observables are invariant under those aperiodic gauge transformations

$$
U(\vec{x}, t+\beta)=z U(\vec{x}, t)
$$

where $z=\exp (2 \pi i n / N)$ is an element of the $Z(N)$ centre of the gauge group. This is not true of the Polyakov loop, which transforms under (8) according to

$$
L \rightarrow z L
$$

Therefore, $\langle L\rangle$ is an order parameter. Going from $\langle L\rangle=0$ to $\langle L\rangle \neq 0$ requires the spontaneous breaking of a symmetry — in this case, spontaneous breaking of $Z(N)$. Hence, the realization of the $Z(N)$ symmetry directly determines whether or not the $S U(N)$ gauge theory is in a confining phase.

The confinement/deconfinement phase transition as a function of temperature in $S U(N)$ gauge theory is a transition in which a global $Z(N)$ symmetry is spontaneously broken. If the order parameter $\langle L\rangle$ varies continuously (but non-analytically) from $\langle L\rangle=0$ for $T<T_{c}$ to $\langle L\rangle \neq 0$ for $T>T_{c}$, then the transition is second order. If, on the other hand, $\langle L\rangle$ is discontinuous at $T_{c}$, the transition is first order. Following Landau, 15 we write an effective Lagrangian for $L$ at long length scales whose terms are determined by the symmetry of the order parameter

$$
S_{\text {eff }}=\int \mathrm{d}^{3} \vec{x}\left[\left(\partial_{i} L\right)^{2}+V(L)\right]
$$


where the effective potential is

$$
V(L)=f\left(|L|^{2}\right)+g\left(|L|^{2}, \operatorname{Re}\left(L^{N}\right)\right) .
$$

In general, $f$ and $g$ are arbitrary functions. To look for a second order phase transition, we expand them about the origin giving

$$
V(L)=a|L|^{2}+b|L|^{4}+c \operatorname{Re}\left(L^{3}\right)+\cdots
$$

where $a, b$, and $c$ are functions of temperature and we have specialized to $S U(3)$. Taking $b>0$, we see that if $c$ were zero, the minimum of the potential would increase continuously from $L=0$ to $L \neq 0$ as $a$ went through zero. However, with the $c$ term present $|L|$ will always jump discontinuously away from zero as $a$ is lowered. In fact, no cases are known in which there is a second order phase transition involving an order parameter whose symmetries allow a cubic invariant in the free energy. The cubic term which is present because the symmetry is $Z(3)$ forces the phase transition to be first order.

The QCD phase transition is an inherently non-perturbative phenomenon. Perhaps the best long term strategy for studying it in full (non-universal) detail from first principles is to use lattice gauge theory. Following Wilson, 16 the theory can be formulated on a lattice and the Euclidian path integral (1) can be evaluated using Monte Carlo techniques 17 This involves evaluating expectation values of operators by importance sampling — one chooses configurations weighted by a known probability measure - and is therefore limited to studying equilibrium situations in which no time evolution occurs. The prediction of Svetitsky and Yaffe described above that the pure $S U(3)$ phase transition is first order has been confirmed in lattice gauge theory simulations 18

Including dynamical quarks in the partition function changes the situation entirely. $\langle L\rangle$ is nonzero even in the confined phase. This can be understood heuristically by noting that a static quark source can always be screened by virtual $q \bar{q}$ pairs in the ensemble. It can also be understood more formally 14 by showing that after integrating out the fermions, the effective action for the gauge fields includes a term which breaks the $Z(N)$ symmetry. No order parameter is known for the confinement/deconfinement transition in $S U(N)$ gauge theory when dynamical fermions are present. This would seem to preclude the existence of a second order transition. A first order transition is logically possible, and presumably is obtained for heavy quarks. For two species of light quarks, on the other hand, lattice gauge theory simulations $19,20,21,22,23,24,25,26,27$, show no signs of a first order deconfinement transition. $L$ increases smoothly as a function of increasing temperature and no discontinuities are apparent. As in the ionization of a gas, there is no sharply defined transition, but rather a smooth change from one regime to another.

Arguing only from fundamental symmetries, we have found that the phase transition is first order in pure gluon QCD. We found an order parameter, but its symmetry precluded a second order transition. In the presence of dynamical quarks, we 
were unable even to find an order parameter. Had we found a second order transition, we could do much more. Powerful renormalization group techniques could be brought to bear on the problem, superceding the mean field analysis above. When there are massless quarks present, while there is no order parameter for the confinement/deconfinement transition, there is an order parameter for the chiral phase transition. In Chapter 2, following arguments first advanced by Pisarski and Wilczek28 which are similar in philosophy to those above, we will discover that for two species of massless quarks the chiral phase transition can be second order. This will have many implications and quantitative consequences.

All the results of Chapter 2 describe quantities observable in thermal equilibrium where there is no time evolution. As we have discussed, this makes them ideal for comparison with lattice "experiments". Working on the lattice, one has more freedom to adjust the parameters of the theory than in the real world. "Experimentalists" can choose what quark masses to use, while experimentalists cannot. We will see that this is unfortunate, because in order to observe the critical phenomena discussed in Chapter 2 on the lattice or in the real world, it will be desirable to use smaller up and down quark masses than nature does. This is possible on the lattice, although difficult in practice in current simulations. The future for "experimentalists" is bright.

What about experimentalists? Experimentalists hope to study the physics of the QCD phase transition in relativistic heavy ion collisions. We will argue in Chapter 2 that if the plasma in these events stays in equilibrium, it is unlikely that the chiral transition will have a dramatic signature. Of course, it is still possible to hope to detect the difference between $T \gg T_{c}$ and $T \ll T_{c}$. It is just that the physics at $T \sim T_{c}$ will be smooth. In studying heavy ion collisions, however, we really should be open to the possibility that the plasma cools through the transition without staying in thermal equilibrium. This will be our concern in Chapter 3. We will find that it is possible that long wavelength oscillations of the pion field are excited, leading to the production of clusters of pions within which the number ratio of neutral pions is far from $1 / 3$. This is a potentially dramatic signature of the chiral phase transition in relativistic heavy ion collisions in which thermal equilibrium is not maintained.

As previously mentioned, the second goal of this introduction is to sketch some standard ideas about what may happen in ultra-relativistic heavy ion collisions. We will not review this enormous field comprehensively. References to reviews wherein the interested reader can find both discussions of greater depth and further references will be given. The reader should also consult other articles in this volume.

Let us recall a few qualitative facts which are known empirically from studying high energy proton-proton collisions with multiple production of secondary particles, 29.30 and which are presumed to apply to high enough energy collisions between nuclei also.31]32 The momentum distribution of the secondary particles which could in general be a function of the longitudinal and transverse momenta of the particles $\left(p_{L}, p_{T}\right)$ and the centre of mass energy of the incident protons $(\sqrt{s})$ is in fact observed to be 
independent of $\sqrt{s}$ for $\sqrt{s}$ larger than about $10 \mathrm{GeV}$ and the invariant cross-section factorizes according to

$$
\frac{E \mathrm{~d}^{3} \sigma}{\mathrm{d}^{3} p}=\frac{E \mathrm{~d}^{3} \sigma}{\mathrm{d}^{2}\left(p_{T}\right) \mathrm{d} p_{L}}=F_{1}(x) F_{2}\left(p_{T}\right) .
$$

$E, \vec{p}$, and $m$ are the energy, momentum and mass of a particular secondary particle. The Feynman $x$ variable is given by

$$
x=\frac{p_{L}}{p_{L \max }} \simeq \frac{2 p_{L}}{\sqrt{s}} .
$$

The transverse momentum distribution $F_{2}$ falls rapidly with increasing $p_{T}$ and the mean transverse momentum is $p_{T} \sim 0.35 \mathrm{GeV} .30$ (Events in which hard parton-parton scatterings give rise to jets with large $p_{T}$ occur about once per million collisions 30 Most high energy proton-proton collisions yield secondary particles with small transverse momenta.) To discuss the longitudinal momentum distribution it is useful to define a variable $y$, called rapidity, where

$$
y \equiv \frac{1}{2} \log \left(\frac{E+p_{L}}{E-p_{L}}\right)=\log \left(\frac{E+p_{L}}{\sqrt{p_{T}^{2}+m^{2}}}\right) .
$$

One reason why $y$ is convenient is that boosting in the beam direction corresponds simply to adding a constant to $y$. Another reason is that for $p \gg m$,

$$
y \simeq \eta \equiv-\log \tan \theta / 2
$$

where the pseudorapidity $\eta$ is easily measured since it depends only on the polar angle $\theta$. A third useful property of $y$ is that

$$
\mathrm{d} y=\frac{\mathrm{d} p_{L}}{E} .
$$

We will work in the centre of mass frame, where $p_{L}=x=0$ corresponds to $y=0$ and $p_{L}= \pm p_{L} \max$ corresponds to $y= \pm y_{\max }$ where

$$
y_{\max }=\frac{1}{2} \log \left(\frac{s}{p_{T}^{2}+m^{2}}\right) .
$$

Feynman postulated 29 that at large $s$ there is a central rapidity region $\left(|y| \ll y_{\max }\right.$, or, equivalently, $\left.\left|p_{L}\right| \ll p_{L \max }\right)$ where $F_{1}(x)$ is a constant. In this region,

$$
E \mathrm{~d}^{3} \sigma=F_{1} F_{2}\left(p_{T}\right) \mathrm{d}^{2}\left(p_{T}\right) \mathrm{d} p_{L}
$$

and, integrating over $p_{T}$ and using (17),

$$
\frac{\mathrm{d} \sigma}{\mathrm{d} y}=\text { constant . }
$$


Thus, there is a central rapidity region in which the multiplicity per unit rapidity is approximately constant. This result has indeed been seen in proton-(anti)proton collisions.30 The qualitative features found in high energy hadron-hadron collisions - low $p_{T}$ and a central rapidity plateau - are expected 31 to characterize ultrarelativistic nucleus-nucleus collisions also.

In a relativistic heavy ion collision of sufficiently high energy, the decay products divide naturally into two regions. In the centre of mass reference frame, the fragments of the incident nuclei are found at high (positive and negative) rapidity, while the central rapidity region is characterized by approximately constant multiplicity per unit rapidity and by low baryon number density. 31 At early times, this central rapidity region experiences boost invariant longitudinal expansion 31 in which the momentum and position of particles are related in such a way that the rapidity is given by

$$
y=\frac{1}{2} \log \left(\frac{t+z}{t-z}\right)
$$

where $t$ is the time and $z$ is the position coordinate in the beam direction. Mean physical quantities depend only on the proper time

$$
\tau \equiv \sqrt{t^{2}-z^{2}}
$$

and transverse position, and are independent of $y$.

Describing the fragmentation regions requires consideration of QCD at nonzero temperature and baryon number density, and is not discussed in this article. We limit ourselves to addressing the physics of the "hot vacuum" in the central rapidity region. How high an energy is sufficient for there to be a reasonably clean separation between distinct fragmentation and central rapidity regions? The parameter of interest is $\delta y$, the extent in rapidity of one of the fragmentation regions. $\delta y$ is not well known - estimates range from 4 to 8.33 Even if $\delta y \sim 4$, present fixed target heavy ion collision experiments at the AGS and SPS do not access sufficient rapidities for there to be a baryon poor central rapidity region. At the AGS, $y_{\max } \sim 2$ and at the SPS $y_{\max } \sim 3$. In the coming generation of colliding beam heavy ion accelerators, the situation will be much improved. At the Relativistic Heavy Ion Collider currently under construction at Brookhaven National Laboratory, it is expected that $\mathrm{Au}-\mathrm{Au}$ collisions with $\sqrt{s}=200 \mathrm{GeV} / \mathrm{A}$ with multiplicities of at least 1400 per unit rapidity will be achieved 33 When the LHC is built at CERN and is run as a $\mathrm{Pb}-\mathrm{Pb}$ collider, collisions with $\sqrt{s}=6300 \mathrm{GeV} / \mathrm{A}$ and with multiplicities of at least 2600 per unit rapidity will be possible. 33 These multiplicity estimates are qualitative - they are based on extrapolating from data from proton-nucleus collisions, and results from event generators suggest that they could be too low 33 At RHIC $\left(y_{\max } \sim 5\right)$ the central rapidity region will have essentially zero baryon number density if $\delta y<5$ and at the LHC $\left(y_{\max } \sim 9\right)$ there will be such a region for $\delta y<933$ Even if there are baryons in the central region at RHIC, the number ratio of mesons to baryons is 
estimated to be $\sim 10^{2} 33$ (At the LHC, this ratio is expected to be $\gg 10^{5} .33$ ) Thus, analyzing the physics of QCD at finite temperature and zero baryon number density will be of relevance in describing the physics of the central rapidity regions of heavy ion collisions at RHIC and the LHC.

What happens in the central rapidity region? The standard description involves three phases - a pre-equilibrium phase, a hydrodynamic evolution phase during which thermal equilibrium is assumed, and a hadronization phase. There have been a number of different approaches to treating the earliest phase of a heavy ion collision. For example, in the string model, one imagines that the incident nuclei pass through each other and colour flux tubes, or strings, are drawn out between the receding fragmented nuclei. These strings then break apart by quark-anti-quark pair production, and realizations of the scenario are based on Monte Carlo simulation of this process. This scenario has been reviewed by Białas and Czyź.34 It runs into difficulties at energies high enough that the string density becomes large. At high enough energies, it should be possible to describe the earliest phase of the evolution in terms of parton interactions by perturbative QCD. This approach, called the parton cascade model, 35, 36.9 has begun to yield results. One starts with distribution functions for the partons in the nucleons of the incident nuclei, and follows the quark-gluon interactions in the framework of renormalization group improved perturbative QCD by solving transport equations for the parton distributions in phase-space with Monte Carlo methods. The results of this work give support at the parton level to the idea that a thermalized state is formed. Quarks thermalize more slowly than gluons because the relevant QCD cross-sections are larger by a factor of two or three 37 Also, for both quarks and gluons, a thermal distribution of momenta is achieved more quickly than chemical equilibrium (equilibrium number density) is achieved. (It is not clear whether there will be enough time in a RHIC collision for quark chemical equilibrium to be established.37,36) It is of interest that at the latest times considered by Geiger $35(\sim 3 \mathrm{fm} / c)$, the baryon number is in fragmentation regions of high rapidity, and the central rapidity region contains partons with thermalized momenta which are expanding longitudinally with little transverse motion. While these calculations are far from the end of the story of the pre-equilibration physics, they do provide justification for supposing that in an energetic enough collision (perhaps at RHIC) there is a low baryon number central rapidity region in which particle momenta are in local thermal equilibrium at $T>T_{c}$.

If equilibrium is achieved, and while it is maintained, the evolution of the plasma can be described hydrodynamically. The matter is treated as a relativistic fluid described by smoothly varying four-velocity, pressure, temperature, and energy density without reference to the microscopic description in terms of partons. This approach has a long history, but perhaps the first quantitative treatment was that of Bjorken. 31 The review by Blaizot and Ollitrault 38 is a useful introduction to the subject. Bjorken 31 used the observation that the central rapidity region is approximately 
boost invariant to find solutions to the hydrodynamic equations describing the longitudinal expansion and consequent cooling of the plasma. Blaizot and Ollitrault 88 review various numerical simulations of the hydrodynamic equations which include transverse expansion.

In using the hydrodynamical approach one assumes that thermal equilibrium is valid throughout the transition and until the hadrons no longer interact. "This is an assumption which is hard to justify in any satisfying way." 39 It is particularly difficult to see how long wavelength degrees of freedom can stay in equilibrium as the plasma expands and cools through the phase transition. Given the uncertainties, in considering the physics of the chiral phase transition in relativistic heavy ion collisions we will discuss both the phenomena that would occur if the plasma stays in equilibrium and those that could characterize a far from equilibrium transition.

In the final phase of a heavy ion collision, regardless of what has happened earlier, hadrons, photons, and leptons impinge upon an experimentalist's detector. How is the experimentalist supposed to unravel what has gone on before? The zeroth order question is how can she determine whether for a brief instant a small region of quarkgluon plasma was created? Many signatures have been proposed for the production of a region of quark-gluon plasma in a heavy ion collision and its consequent passage through the QCD phase transition. All have problems to overcome, arising either from background, or from insufficient theoretical understanding, or from the possibility that the signature can be mimicked by hadronic processes. This suggests that the QCD phase transition will be detected by a process where various pieces of evidence become more and more convincing rather than by the observation of a "smoking gun" signature. If the speculations of Chapter 3 are correct, however, the situation could be improved. We will see that if the chiral phase transition occurs by a process that is far enough from thermal equilibrium that it can be idealized as a quench, then coherent long wavelength oscillations of the pion field may emerge. These would manifest themselves as clusters of pions with similar rapidity in which the number ratio of neutral pions is dramatically different from $1 / 3$. If seen, this phenomenon would be a distinctive, qualitative signature that a far from equilibrium chiral transition had occurred.

\section{Critical Phenomena at a Second Order QCD Phase Transition}

The QCD phase transition has been studied from many different perspectives using many different models and approximations. Here, however, we will attempt to learn as much as can be learned analytically about the phase transition, relying as much as possible only on fundamental symmetries and universality arguments and as little as possible on specific assumptions and models. This philosophy yields little fruit when applied to the confinement/deconfinement transition. We saw in the previous chapter that in QCD with light quarks, this transition is neither first order nor second order 
- it is a smooth crossover like the ionization of a gas. The chiral phase transition is another story. Wilczek $\omega^{0}$ has emphasized that in the chiral limit where there are two species of quarks with zero current algebra mass, the order parameter for the chiral phase transition has the same symmetry as the magnetization of a four component Heisenberg magnet, which has a second order phase transition. At an equilibrium second order phase transition, thermodynamic quantities have non-analytic behaviour determined by the physics of the modes which develop long correlation lengths at the critical point. Thus, by studying these universal characteristics in a much simpler model — the $N=4$ Heisenberg magnet - it is possible to learn much about the chiral phase transition in QCD.

In this chapter, which to a large extent follows work done with Wilczek, 自 we explore the consequences of this approach to the QCD phase transition in thermal equilibrium. In the following section, we use renormalization group arguments to establish a dictionary between QCD and the magnetic system. Using this dictionary, we apply results for the static critical exponents obtained in the magnet model to QCD. In section 2.2, we discuss the behaviour of the pion and sigma masses at the transition. In section 2.3, we review recent work in lattice gauge theory which has begun to test these ideas. In section 2.4, we consider how the strange quark affects the phase transition, and again compare with lattice results. In the concluding section of this chapter, we discuss the implications of all this for cosmology and lattice gauge theory, and for heavy ion collisions under the assumption that the chiral order parameter remains close to thermal equilibrium through the phase transition.

\section{1. $Q C D$ and the $O(4)$ Magnet}

The physics of the QCD phase transition is qualitatively different in the cases of zero, one, two, or three or more flavours of quarks. 10 In this section we consider

QCD with two species of quarks. Pisarski and Wilczek28 used an analysis similar to the one which follows to show that for three or more flavours of massless quarks, the chiral phase transition is first order. In section 2.4 we will discuss the effects of a third quark species, massless or massive, and for now, therefore, we postpone our discussion of the effects of the strange quark. If there are two flavours of massless quarks, the QCD lagrangian is symmetric under global chiral transformations in the group $S U(2)_{L} \times S U(2)_{R} \times U(1)_{L+R}$ of independent special unitary transformations of the left and right handed quark fields, and a vector $U(1)$ transformation which corresponds to baryon number symmetry. (The axial $U(1)$ which would make the symmetry group into $U(2) \times U(2)$ is a symmetry of the classical theory, but not of the quantum theory.11) This chiral symmetry breaks spontaneously down to $S U(2)_{L+R} \times U(1)_{L+R}$ at low temperatures, and is restored at sufficiently high temperatures. The order parameter for this phase transition is the expectation value of the quark bilinear

$$
\mathcal{M}_{j}^{i} \equiv\left\langle\bar{q}_{L}^{i} q_{R j}\right\rangle
$$


which breaks the symmetry when it acquires a nonzero value below some critical temperature $T_{c}$.

At a second order phase transition, the system is at an infrared fixed point of the renormalization group. Renormalization group transformations correspond to coarsening one's view of the world. Thus, at an infrared fixed point, physics is scale invariant. The order parameter fluctuates on all length scales and, in particular, on arbitrarily long length scales. The correlation length associated with these scale invariant fluctuations is necessarily infinite, and the correlation function which describes them is a power law. Our goal is not to describe all of the physics occurring at the transition. Rather, we limit our attention to the nonanalyticities of thermodynamic quantities. The partition function $Z=\operatorname{Tr} e^{-H / T}$ is the sum of analytic terms, and so in a system with finitely many degrees of freedom must be an analytic function of the temperature. Nonanalyticity can only arise in the infinite volume limit, and so by limiting the scope of our treatment in this way we concentrate on the physics of the long wavelength degrees of freedom. At an infrared fixed point, renormalization (i.e. coarsening one's view) does not change the physics of the long wavelength fluctuations of the order parameter, which is therefore independent of all short wavelength physics. The nonanalytic critical phenomena are universal: they only depend on the modes with long wavelength fluctuations (and these are determined by the symmetries of the order parameter) and on the scaling behaviour near the fixed point of low dimension operators constructed from these modes.

In order to describe a second-order transition quantitatively, we must find a tractable model in the same universality class. For the chiral order parameter (23) the relevant symmetries are independent unitary transformations of the left- and right-handed quark fields, under which

$$
\mathcal{M} \rightarrow U^{\dagger} \mathcal{M} V
$$

These transformations generate an $U(2)_{L} \times U(2)_{R}$ symmetry, which is not quite what is needed, since it includes the axial baryon number symmetry which is not present in QCD. This problem is solved 40 by restricting $\mathcal{M}$ to multiples of unitary matrices with positive determinant, instead of general complex matrices. Matrices $\mathcal{M}$ in this restricted class remain in this restricted class under the transformation (24) only if $U$ and $V$ have equal phases. Hence, the axial $U(1)$ has indeed been removed. The $2 \times 2$ matrices $\mathcal{M}$ can conveniently be parametrized using four real parameters $(\sigma, \vec{\pi})$ and the Pauli matrices as

$$
\mathcal{M}=\sigma+i \vec{\pi} \cdot \vec{\tau}
$$

In fact the order parameter can be written as a four-component vector

$$
\phi^{\alpha} \equiv(\sigma, \vec{\pi})
$$

and the transformations (24) are simply $O(4)$ rotations in internal space. Hence, the order parameter appropriate for the chiral phase transition in QCD with two 
flavours of massless quarks has the symmetries of the standard $O(4)$ invariant $N=$ 4 Heisenberg magnet. For smaller number of components, this sort of model is a much-studied model for the critical behaviour of magnets, with the order parameter representing the magnetization of a ferromagnet or the staggered magnetization of an antiferromagnet.

In order to discuss the universal critical phenomena, it is sufficient to retain those degrees of freedom which develop large correlation lengths at the critical point. These are just long wavelength fluctuations of the order parameter, which is small in magnitude near the critical point and therefore fluctuates at little cost in energy. Thus, the most plausible starting point for analyzing the critical behaviour of a second-order chiral phase transition in QCD is the Landau-Ginzburg free energy

$$
F=\int d^{3} x\left\{\frac{1}{2} \partial^{i} \phi^{\alpha} \partial_{i} \phi_{\alpha}+\frac{\mu^{2}}{2} \phi^{\alpha} \phi_{\alpha}+\frac{\lambda}{4}\left(\phi^{\alpha} \phi_{\alpha}\right)^{2}\right\} .
$$

Here $\mu^{2}$ is the temperature dependent renormalized mass squared. $T_{c}$ is the temperature at which $\mu^{2}$ vanishes. $\mu^{2}$ is positive above the critical temperature and negative below it. The quartic coupling $\lambda$ depends smoothly on the temperature. In this section we consider the case in which $\lambda$ is positive; we will discuss what happens if $\lambda$ is not positive at $T_{c}$ in section 2.4. We neglect higher dimension operators, because they are irrelevant at an infrared fixed point - their coefficients go to zero upon renormalization toward the infrared. ${ }^{a}$ The symmetry breaking pattern we want is $\langle\mathcal{M}\rangle \propto \mathbf{1}$ (equivalently, $\langle\sigma\rangle \neq 0 ;\langle\vec{\pi}\rangle=0$ ) below the transition which is indeed what we find at the minimum of the potential for positive $\lambda$. This model has been studied in depth for arbitrary $N$ and spatial dimension $d$, and the existence of an infrared stable fixed point of the renormalization group has been established 42 Hence, it is a model for a second order QCD chiral phase transition for two massless quarks.

Before proceeding, let us briefly outline the the physics of the theory given by (27) in thermal equilibrium above, below, and at the critical temperature at which the second order phase transition occurs. At temperatures above $T_{c}$, the direction in internal space in which $\phi$ is oriented is correlated only over spatial distances of order a finite correlation length; over longer distances all orientations of $\phi$ are equally probable. Thus, $\phi$ is disordered and $\langle\phi\rangle=0$. At temperatures below $T_{c}, \phi$ is ordered: $\langle\phi\rangle$ is nonzero and selects a particular direction (defined to be the $\sigma$ direction) in internal space. Whereas the theory has an explicit $O(4)$ symmetry, the equilibrium configuration is not $O(4)$ symmetric - the symmetry is spontaneously broken. Fluctuations of $\phi$ about $\langle\phi\rangle$ in the $\sigma$ and $\vec{\pi}$ directions are described by differing correlation functions. The explicit $O(4)$ symmetry implies that $\langle\phi\rangle$ could choose any direction in internal space. Below $T_{c}$, therefore, fluctuations of $\phi$ in the $\vec{\pi}$ directions (which correspond to fluctuations of the orientation of $\langle\phi\rangle)$ are massless Goldstone modes.

${ }^{a} \phi^{6}$ is not irrelevant in 3-dimensions, but as long as $\lambda$ is positive at $T_{c}, \phi^{6}$ can be neglected relative to $\phi^{4}$ since $|\phi|$ is small near the transition. The $\phi^{6}$ term will be important in section 2.4. 
At $T=T_{c},\langle\phi\rangle=0$, the correlation length is infinite, and the fluctuations of $\phi$ are scale invariant.

We have established that there is an infrared fixed point of the renormalization group in the same universality class as QCD with two massless quarks. The theories in this universality class live in an infinite dimensional parameter space spanned by $\mu$, $\lambda$, and the coefficients of an infinite number of irrelevant higher dimension operators. The second order fixed point has some basin of attraction in this space of theories. For the rest of this chapter, we will explore the hypothesis that QCD is indeed in the basin of attraction of the infrared fixed point. We will calculate much about the QCD phase transition based on this assumption. We will also be show that it is consistent with many phenomena found to date in lattice gauge theory simulations. We can never be sure, however, that upon renormalization toward the infrared QCD is in fact driven to this fixed point. It is always a logical possibility that, say, QCD may live in a region of theory space from which infrared renormalization drives the theory into a region of negative $\lambda$, making the phase transition first order. Hence, the strongest statement that it is possible to make is that it is plausible that in QCD with two species of massless quarks the chiral phase transition is second order. Our strategy, then, is to establish quantitative consequences of this hypothesis and test them against lattice simulations of QCD.

When the free energy (27) is written in terms of $\sigma$ and $\vec{\pi}$ it looks much like the original model of Gell-Mann and Lévy 43 with two changes: there are no nucleon fields and only three (spatial) dimensions. These two changes reflect an important distinction. 4 We are only proposing (27) as appropriate near the second order phase transition point. This is because it is only there that we can appeal to universality the long-wavelength behaviour of the $\sigma$ and $\vec{\pi}$ fields is determined by the infrared fixed point of the renormalization group, and microscopic considerations are irrelevant to it. In Euclidean field theory at finite temperature, the integral over $\omega$ of zero temperature field theory is replaced by a sum over Matsubara frequencies $\omega_{n}$ given by $2 n \pi T$ for bosons and $(2 n+1) \pi T$ for fermions with $n$ an integer. Hence, one is left with a Euclidean theory in three spatial dimensions with massless fields from the $n=0$ terms in the boson sums and massive fields from the rest of the boson sums and the fermion sums. Hence, to discuss the massless modes of interest at the critical point, (27) is sufficient and we do not need to introduce nucleon fields or constituent quark fields. In arriving at an effective three dimensional theory of the long wavelength fluctuations of the order parameter near $T_{c}$, all other bosonic degrees of freedom and all fermionic degrees of freedom have been integrated out.

We have motivated a very definite hypothesis for the nature of the phase transition for QCD with two species of massless quarks, namely that it is in the universality class of the $N=4$ Heisenberg magnet. This means that under renormalization toward the infrared, QCD with two massless quarks is driven to the infrared fixed point of (27) and that therefore the nonanalytic behaviour of thermodynamic quantities near $T_{c}$ is 
the same in the two theories. This hypothesis has numerous consequences which are the subject of the rest of this chapter. In the remainder of this section, we review the resulting predictions for the critical exponents. 4

First, we define the reduced temperature $t=\left(T-T_{c}\right) / T_{c}$. The exponents $\alpha$, $\beta, \gamma, \eta$, and $\nu$ describe the singular behaviour of the theory with strictly zero quark masses as $t \rightarrow 0$. For the specific heat one finds

$$
C(T) \sim|t|^{-\alpha}+\text { less singular. }
$$

The behaviour of the order parameter defines $\beta$.

$$
\langle|\phi|\rangle \sim|t|^{\beta} \text { for } t<0
$$

$\eta$ and $\nu$ describe the behaviour of the correlation length $\xi$ where

$$
G_{\alpha \beta}(x) \equiv\left\langle\phi(x)_{\alpha} \phi(0)_{\beta}\right\rangle-\left\langle\phi_{\alpha}\right\rangle\left\langle\phi_{\beta}\right\rangle \rightarrow \delta_{\alpha \beta} \frac{A}{|x|^{(d-2+\eta)}} \exp (-|x| / \xi) \text { for }|x| \rightarrow \infty .
$$

$A$ is independent of $|x|$, but may depend on $t$. The correlation length diverges as $|t| \rightarrow 0$, and the correlation length exponent $\nu$ is defined by

$$
\xi \sim|t|^{-\nu}
$$

Above $T_{c}$, where the correlation lengths are equal in the sigma and pion channels, the susceptibility exponent $\gamma$ is defined by

$$
\int d^{3} x G_{\alpha \beta}(x) \sim t^{-\gamma}
$$

We will discuss the behaviour of the susceptibility below the transition in the following section. At the critical point, the correlation length is infinite and the correlation function is a power law. The exponent $\eta$ is defined through the behaviour of the Fourier transform of the correlation function at $t=0$ :

$$
G_{\alpha \beta}(k \rightarrow 0) \sim k^{-2+\eta}
$$

The last exponent, $\delta$, is related to the behaviour of the system in a small magnetic field $H$ which explicitly breaks the $O(4)$ symmetry. Let us first show that in a QCD context, $H$ is proportional to a common quark mass $m_{u}=m_{d} \equiv m_{q}$. This common mass term may be represented by a $2 \times 2$ matrix $\mathcal{D}$ given by $m_{q}$ times the identity matrix. We are now allowed to construct the free energy from invariants involving both $\mathcal{D}$ and $\mathcal{M}$. The lowest dimension term linear in $\mathcal{D}$ is just $\operatorname{tr} \mathcal{M}^{\dagger} \mathcal{D}=m_{q} \sigma$, which in magnet language is simply the coupling of the magnetization to an external magnetic field $H \propto m_{q}$. In the presence of an external field, the order parameter is not zero at $T_{c}$. In fact,

$$
\langle|\phi|\rangle(t=0, H \rightarrow 0) \sim H^{1 / \delta}
$$


Thus when the $O(4)$ symmetry is explicitly broken by a small external magnetic field or equivalently by small quark masses, the phase transition is a smooth crossover. It is, however, a very special crossover about which much can be said, because as $H \rightarrow 0$ one approaches the second order fixed point.

The six critical exponents defined above are related by four scaling relations. 2 These are

$$
\begin{aligned}
\alpha & =2-d \nu \\
\beta & =\frac{\nu}{2}(d-2+\eta) \\
\gamma & =(2-\eta) \nu \\
\delta & =\frac{d+2-\eta}{d-2+\eta} .
\end{aligned}
$$

We therefore need values for $\eta$ and $\nu$ for the four component magnet in $d=3$. These were obtained in the remarkable work of Baker, Meiron and Nickel, 4 who carried the perturbative expansion in $\lambda$ for the theory (27) to seven-loop order, and used information about the behaviour of asymptotically large orders, and conformal mapping and Padé approximant techniques to obtain

$$
\begin{aligned}
& \eta=.03 \pm .01 \\
& \nu=.73 \pm .02
\end{aligned}
$$

Using (35), the remaining exponents are

$$
\begin{aligned}
& \alpha=-0.19 \pm .06 \\
& \beta=0.38 \pm .01 \\
& \gamma=1.44 \pm .04 \\
& \delta=4.82 \pm .05 .
\end{aligned}
$$

Since $\alpha$ is negative there is a cusp in the specific heat at $T_{c}$, rather than a divergence. There are other ways in which these exponents could be calculated. In $d=4$, mean field theory suffices and one has $\eta=0$ and $\nu=1 / 2$. We are interested in $d=3$, however. A standard approach 6 is to work in $4-\epsilon$ dimensions, evaluate the exponents order by order in $\epsilon$, and then set $\epsilon=1$. The $\epsilon$-expansion has not been pushed to high enough order to compete in accuracy with the work of Baker, Meiron, and Nickel. Another approach is to do a finite temperature lattice simulation of the theory (27) and measure the critical exponents. This has recently been done to high accuracy by Kanaya and Kaya, 47 who find $\nu=0.748 \pm 0.009$ and $\gamma=1.48 \pm .02$, in agreement with the results of Ref. 455.

To reiterate, these exponents and the other critical phenomena we discuss in subsequent sections are universal. Whether the QCD phase transition is studied experimentally, or on the lattice, or by instanton liquid techniques, or by using the 
Nambu-Jona-Lasinio model, or by using the (four dimensional) linear or nonlinear sigma models, or by any other means, if the hypothesis that the chiral transition is a second order transition in the $O(4)$ universality class is correct the exponents should turn out to have the values we have described. Of course, using any technique except appealing to the simplest model in the same universality class as we have done would make it much harder to study the theory near the critical point, and hence much harder to obtain the critical exponents.

\subsection{The Equation of State and the Pion and Sigma Masses}

The expressions which define $\beta, \gamma$ and $\delta$ are actually special cases of a more general relationship between the magnetization and the magnetic field called the critical equation of state. The equation of state has been calculated to order $\epsilon^{2}$ by Brézin, Wallace and Wilson. 6 In this section, we review the use of the equation of state to determine the behaviour of the pion and sigma masses near the critical point.

First, we must define what we mean by the "mass" of the pion and sigma. We could choose either to define the mass as an inverse correlation length or as an inverse susceptibility. We choose the latter, which is conventional in the condensed matter literature. Specifically, we define

$$
m_{\sigma}^{-2}=\int d^{3} x G_{00}
$$

and

$$
m_{\pi}^{-2} \delta_{i j}=\int d^{3} x G_{i j}
$$

where $\phi_{0}=\sigma$ and $\phi_{i}=\pi_{i}, i=1,2,3$. Defining "masses" as inverse correlation lengths would give different scaling behaviours for masses as functions of $t$ and $H$. (For $\eta=0$, however, both choices would yield the same scaling behaviour, and in the theory of interest $\eta$ is small.) We shall see that with the conventional choices (38) and (39), the masses can be extracted conveniently from the equation of state. It is worth noting that the masses we have defined are related only to the behaviour of spatial correlation functions in the static (equilibrium) theory. They carry no dynamical information, and should not be confused with, say, pole masses in a 4-dimensional theory. Also, we will only be able to make universal statements about how the masses scale at the transition. Normalizing the magnitudes of the masses will require using some specific model, and hence will not be universal.

The equation of state gives the magnetization as a function of $t$ and $H$. For the rest of this chapter, we will write the order parameter as $M$, for magnetization, keeping in mind that $M=\langle\sigma\rangle=\langle|\phi|\rangle$. Also, recall that $H$, the external field, is proportional to the quark mass $m_{q}$. In order to define the equation of state, we first define a shifted field $\tilde{\sigma}=\sigma-\langle\sigma\rangle=\sigma-M$. Then the equation of state is simply the relation

$$
\langle\tilde{\sigma}\rangle=0
$$




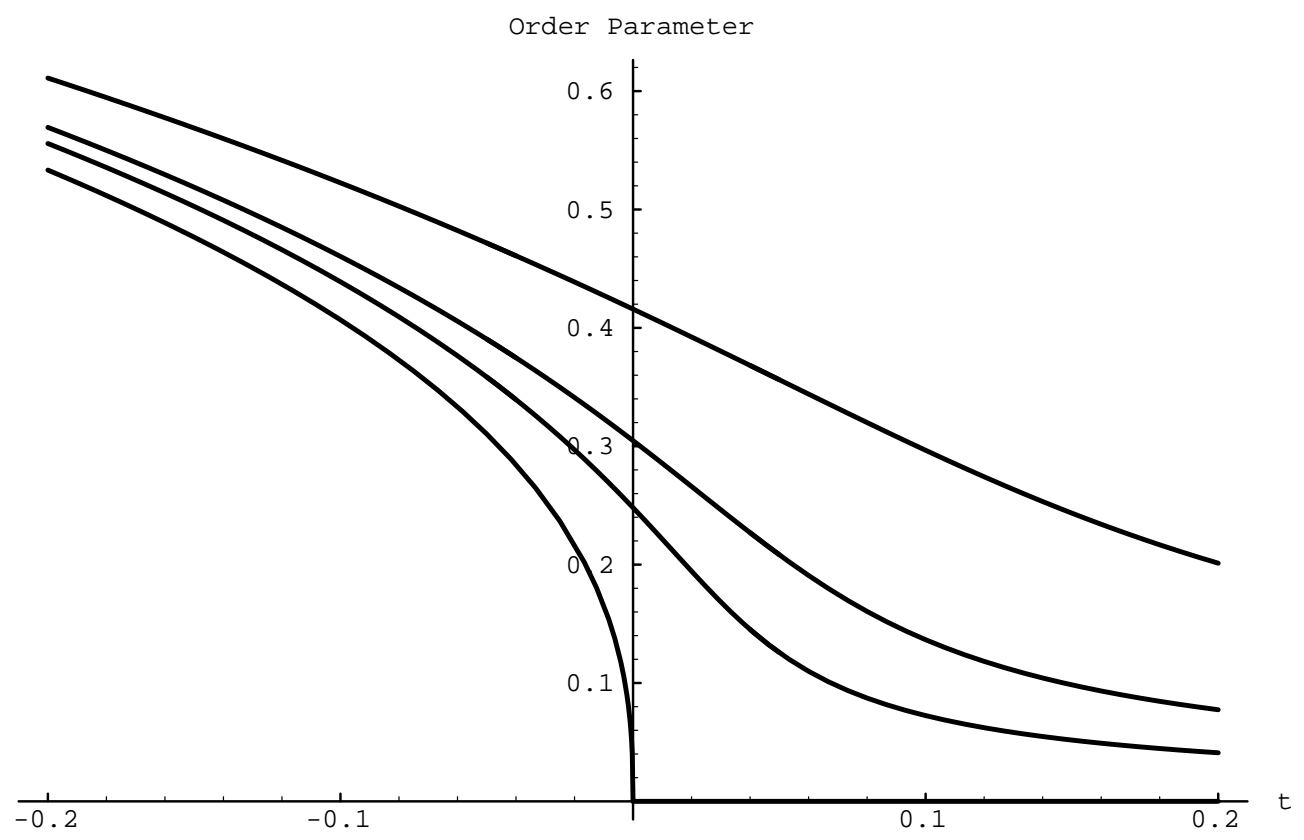

Fig. 1. From Ref. [3]. The order parameter $M$ as a function of reduced temperature $t \equiv\left(T-T_{c}\right) / T_{c}$ for magnetic fields $H=0,0.002,0.005$, and 0.02 . Like $t$, both $M$ and $H$ are dimensionless. They are obtained from their dimensionful counterparts by dividing by non-universal dimensionful constants defined in such a way that for $t<0$ and $H=0$ the order parameter is given by $M=(-t)^{\beta}$, and for $t=0$ it satisfies $M=H^{1 / \delta}$.

This relation has been expanded to order $\epsilon^{2}$ by Brézin, Wallace and Wilson. 19 The result can be expressed conveniently in terms of the variables

$$
y \equiv H / M^{\delta} \text { and } x \equiv t / M^{1 / \beta}
$$

as

$$
y=f(x) .
$$

The function $f(x)$ is universal and was calculated to order $\epsilon^{2}$ by Brézin, Wallace, and Wilson 48 Their result is also quoted in Ref. [3]. The units in which $H$ and $M$ are measured are chosen so that $f(0)=1$ corresponds to $t=0$ and $f(-1)=0$ corresponds to the $t<0, H=0$ coexistence curve. Knowing $f(x)$, we can calculate the value of the order parameter $M$ for a given $H$ and $t$ using (42). The behaviour of the order parameter is illustrated in Fig. 1. This figure and the other ones in this section should be viewed as illustrations of qualitative behaviour rather than quantitative predictions because they are based on setting $\epsilon=1$ in the $O\left(\epsilon^{2}\right)$ expression for $f(x)$. The values for the critical exponents themselves which we quoted in the previous section are quantitative predictions, complete with error estimates, because they are based on the much more elaborate analysis of Baker, Meiron and Nickel. 15 
From the equation of state, we can deduce the behaviour of $m_{\pi}$ and $m_{\sigma}$ at nonzero (but small) $t$ and $H$. The masses are given by

$$
m_{\sigma}^{2}=\frac{\partial H}{\partial M}
$$

and

$$
m_{\pi}^{2}=\frac{H}{M}
$$

The first relation follows directly from the definition (38), and the second follows from (39) and from assuming that $\vec{M} \| \vec{H}$, so that a small change $\delta H \perp H$ gives a small change $\delta M \perp M$ with $\delta M / \delta H=M / H$. Using the equation of state, we can rewrite (43) and (44) as

$$
m_{\pi}^{2}=M^{\delta-1} f(x)
$$

and

$$
m_{\sigma}^{2}=M^{\delta-1}\left(\delta f(x)-\frac{x}{\beta} f^{\prime}(x)\right) .
$$

Hence, $\delta$ can be determined by measuring the ratio $m_{\sigma}^{2} / m_{\pi}^{2}$ at $t=0$. In general, from $f(x)$ we can find the pion and sigma masses for any $t$ and $H$.

There are two interesting limits which we will consider explicitly. First, for $t>0$ and $H \rightarrow 0$ which corresponds to $x \rightarrow \infty$, we should find the full $O(4)$ symmetry, and hence should find that the pion and sigma masses are identical. For $x \rightarrow \infty$, the function $f(x)$ behaves as $f(x)=c x^{\gamma}$. The constant $c$ is given to $O(\epsilon)$ in Ref. [3]. Applying (45) and (46), we find that

$$
m_{\sigma}^{2}=m_{\pi}^{2}=c t^{\gamma} \text { for } x \rightarrow \infty
$$

consistent with the symmetry.

We can also consider the limiting case of approaching the coexistence curve. This means taking $t<0$ and $H \rightarrow 0$, which implies $x \rightarrow-1$. In this limit, $M$ tends to a nonzero constant, and so from (44), we obtain $m_{\pi}^{2} \propto H$, a familiar result for Goldstone bosons. The behaviour of the pion mass is illustrated in Fig. 2.

The result (44) may look peculiar to a particle physicist who is more familiar with the zero temperature result

$$
m_{\pi}^{2}=\frac{2 m_{q}\langle\bar{q} q\rangle}{f_{\pi}^{2}}
$$

Before considering the sigma mass, we therefore pause here to explain how (48) and (44) are related. We have seen that $m_{q} \sim H$ and that the order parameter $\langle\bar{q} q\rangle \sim$ $\langle\sigma\rangle \sim M$. At zero temperature, $f_{\pi}$ is defined in terms of the axial current by the relation

$$
\left\langle 0\left|A_{\mu}^{\alpha}(0)\right| \pi^{\beta}(q)\right\rangle=i f_{\pi} q_{\mu} \delta^{\alpha \beta}
$$




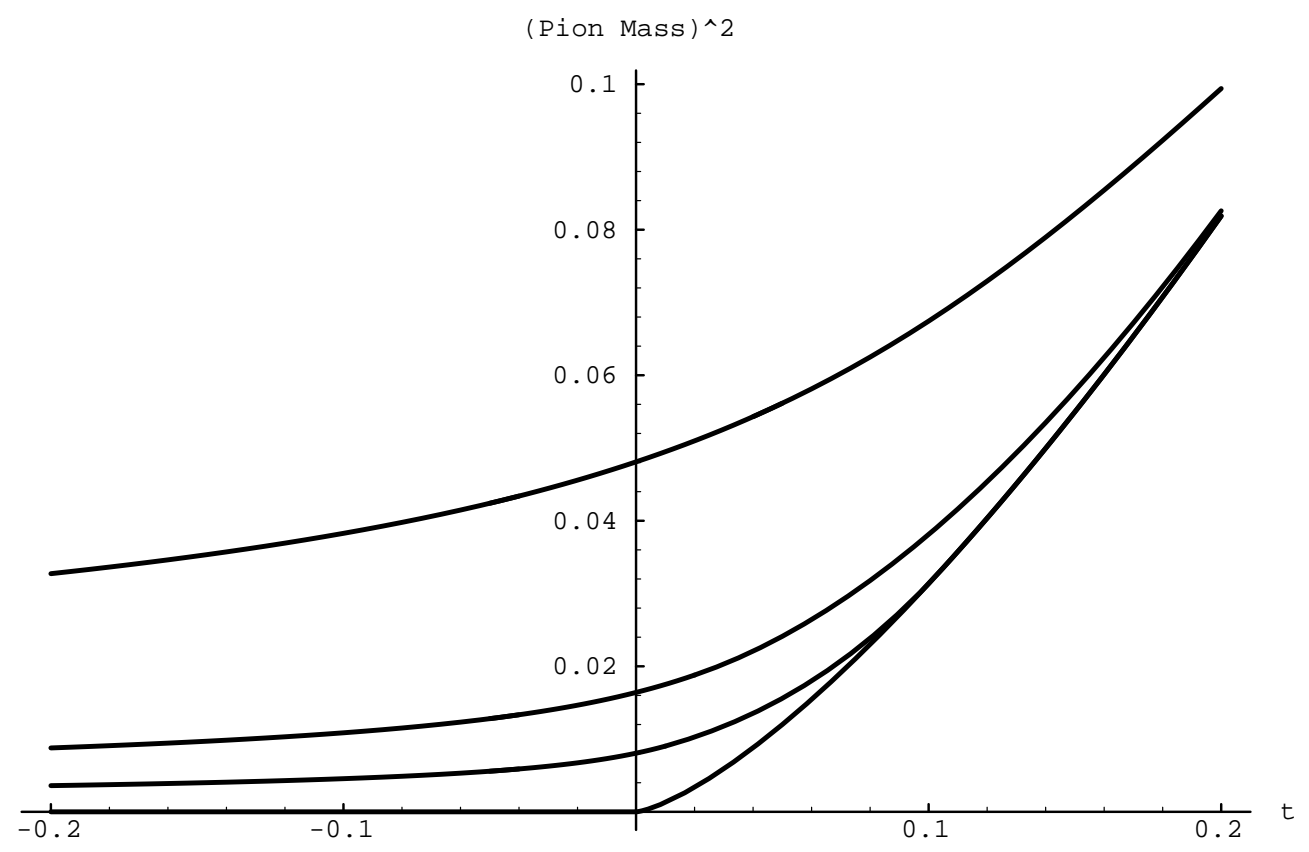

Fig. 2. From Ref. [3]. $m_{\pi}^{2}$ as a function of $t$ for $H=0,0.002,0.005$, and 0.02 . Since $M$ and $H$ are in dimensionless scaled units, so is $m_{\pi}^{2}$. For $t=0, m_{\pi}^{2}=H^{(\delta-1) / \delta}$. For $H=0$ and $t>0$ (and for large enough $t$ for any $H) m_{\pi}^{2} \sim t^{\gamma}$. For $t<0$ and $H \rightarrow 0, m_{\pi}^{2} \sim H$.

In the zero temperature linear sigma model, the axial current is given by

$$
A_{\mu}^{\alpha}(x)=\sigma(x) \partial_{\mu} \pi^{\alpha}(x)-\pi^{\alpha}(x) \partial_{\mu} \sigma(x)
$$

which means that $f_{\pi}$ defined in (48) is simply

$$
f_{\pi}=\langle 0|\sigma| 0\rangle \text {. }
$$

This result suggests that we make the identification $f_{\pi} \sim M$, which does indeed make (44) and (48) equivalent. However, it is important to remember that using the linear sigma model at zero temperature cannot be justified by a universality argument in the way that using it near $T=T_{c}$ can. Hence the argument of this paragraph is not a derivation of (44) from the zero temperature result (48) . (44) is valid near $T=T_{c}$ while (48) is valid at $T=0$. Also, $m_{\pi}$ in (48) is a mass in a $3+1$ dimensional Lorentz invariant theory, while $m_{\pi}^{2}$ in (44) is an inverse susceptibility in a 3 dimensional theory. We have simply shown that a reader familiar with one expression should not be surprised by the other.

The behaviour of the sigma mass at the coexistence curve is trickier to obtain than that of the pion mass. First, we note that in mean field theory $(\epsilon=0)$ the 


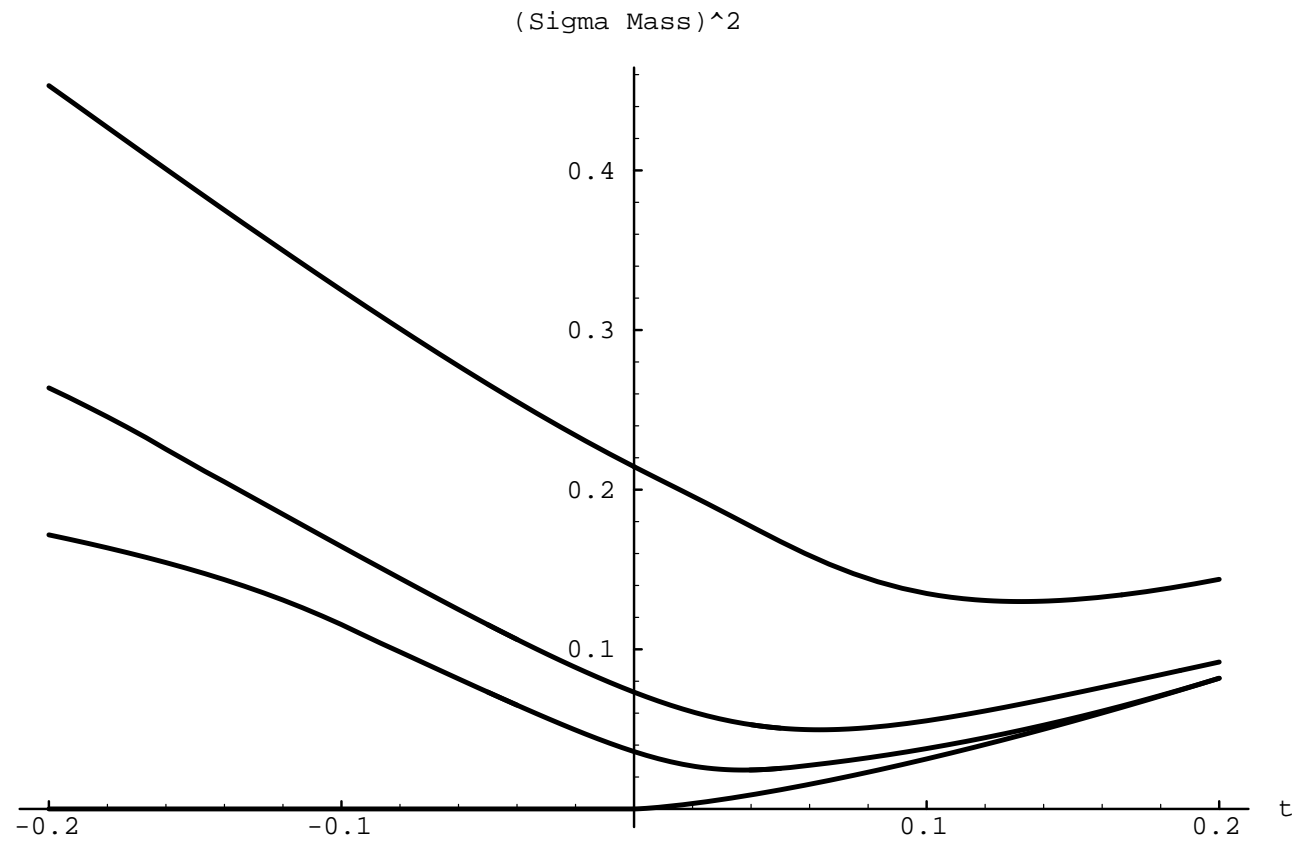

Fig. 3. From Ref. [3]. $m_{\sigma}^{2}$ as a function of $t$ for $H=0,0.002,0.005$, and 0.02. Since $M$ and $H$ are in dimensionless scaled units, so is $m_{\sigma}^{2}$. For $t=0, m_{\sigma}^{2}=\delta m_{\pi}^{2}$. The scale on the vertical axis is different than in Fig. 2. For $H=0$ and $t>0$ (and for large enough $t$ for any $H$ ) $m_{\sigma}^{2}=m_{\pi}^{2}$, and both increase like $t^{\gamma}$. For $t<0$ and $H \rightarrow 0$, the sigma mass decreases to zero as shown in Fig. 4 .

equation of state is simply $y=f(x)=1+x$, and $m_{\sigma}$ is easily evaluated using (46) . For $H \rightarrow 0$ at fixed $t<0$ the result is

$$
m_{\sigma}^{2}=\left(\frac{\delta}{|t|^{\beta}}\right) H+\frac{|t|^{\beta(\delta-1)}}{\beta} .
$$

Hence, in mean field theory $m_{\sigma}^{2}$ decreases with $H$ to a nonzero value at $H=0$. However, for $d<4$ when fluctuations are important, the result is quite different. In words, fluctuations of the massless pions produce new infrared singularities in the longitudinal susceptibility, or, equivalently, make the sigma massless. Now, let us see how this result can be obtained from the equation of state. 99.50 In the limit $H \rightarrow 0$, $f(x) \sim H$ while $f^{\prime}(x)$, we will see, tends to zero more slowly. Hence, the second term in (46) is dominant and gives

$$
\frac{\beta m_{\sigma}^{2}}{M^{\delta-1}} \rightarrow f^{\prime}(x) \text { for } x \rightarrow-1
$$

The difficulty is that for $x \rightarrow-1, f^{\prime}(x)$ contains divergent terms like $\epsilon \log (x+1)$, $\epsilon^{2} \log ^{2}(x+1)$ and $\epsilon^{2} \log (x+1)$. These terms do not exponentiate to $f^{\prime}(x) \sim(x+1)^{p}$. 


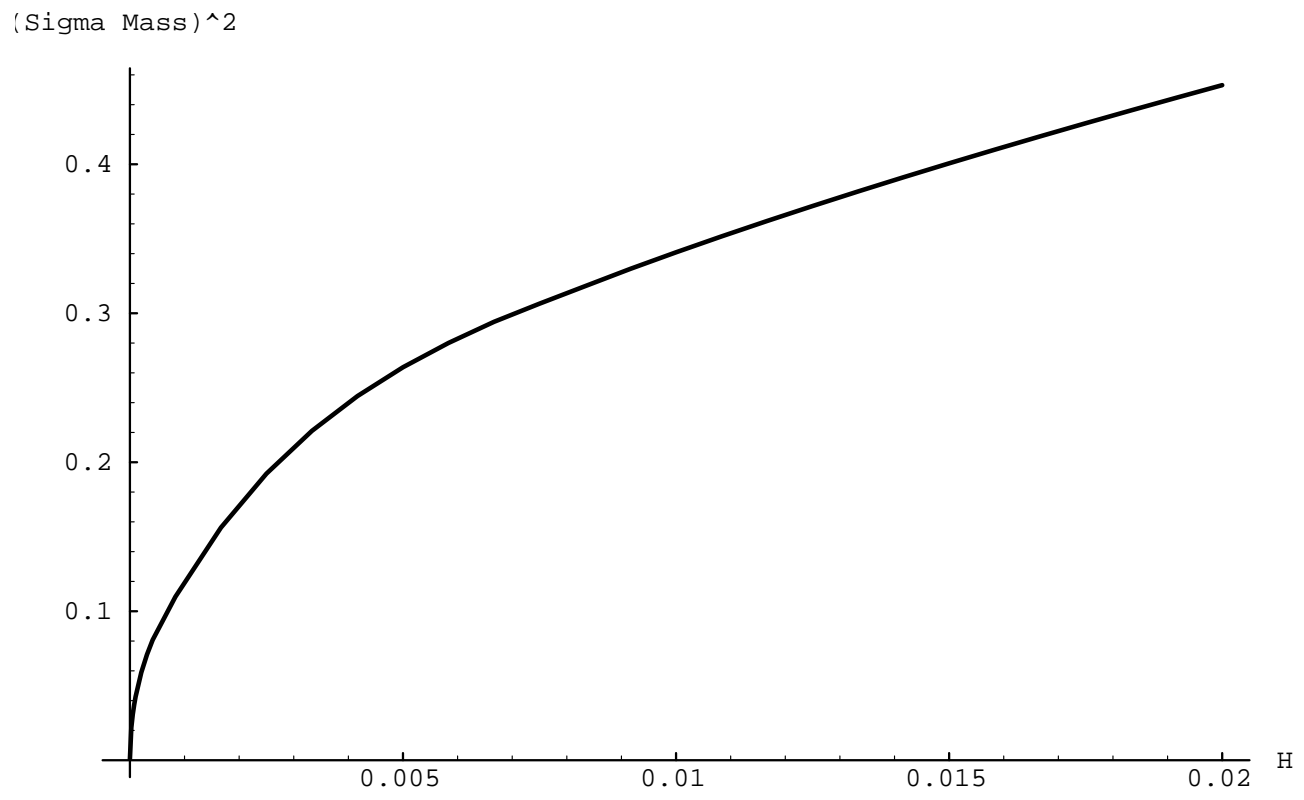

Fig. 4. From Ref. [3]. $m_{\sigma}^{2}$ as a function of $H$ for $t=-0.2$. For large $H$ it behaves as if it will be nonzero at $H=0$, but in fact for $H \rightarrow 0$ it decreases like $m_{\sigma}^{2} \sim H^{p}$ where to lowest order in $\epsilon$, $p=\epsilon / 2=1 / 2$.

Wallace and Zia 50 in fact find the result

$$
\left(\frac{\beta m_{\sigma}^{2}}{M^{\delta-1}}\right)^{-1} \rightarrow c_{1}+c_{2} y^{-\epsilon / 2}
$$

Both the terms on the right side of (54) must be kept because they differ in their exponents only by order $\epsilon$. Also for this reason, the constants $c_{1}$ and $c_{2}$ calculated by Wallace and Zia 50 and given in Ref. [3] are only known to order $\epsilon$ even though $f(x)$ is known to order $\epsilon^{2}$.

Qualitatively, as $H$ is lowered at fixed $t<0$, at first the $c_{1}$ term dominates and $m_{\sigma}^{2}$ appears to be decreasing toward a nonzero value at $H=0$ as in the mean field result. Then, the $c_{2}$ term takes over and one finds that in fact the sigma mass goes to zero like $m_{\sigma}^{2} \propto H^{\epsilon / 2}$. Recently, Anishetty et al. . $^{2}$ have done an extensive analysis of the divergence of the sigma susceptibility produced by the massless pions for $t<0$ and $H \rightarrow 0$. In agreement with the $\epsilon$-expansion result, they find that in $d=3, m_{\sigma}^{2} \propto H^{1 / 2}$. The behaviour of the sigma meson mass is illustrated in Fig. 3 and Fig. 4. In future lattice simulations, as $m_{q}$ is lowered toward zero, this behaviour should be observed. This result is an example of the power of the renormalization group techniques in obtaining universal results. If we had chosen a specific microscopic model, say that of 
Gocksch 52 or the Nambu-Jona-Lasinio model of Hatsuda and Kunihiro, 53 we would have been able to calculate non-universal quantities far from $T_{c}$, but would basically have been limited to using mean field theory, as those authors do. Then, we would have reached the incorrect conclusion that $m_{\sigma} \neq 0$ in the chiral limit below $T_{c}$. Here, by restricting ourselves to calculating universal quantities, we are limited to the region near the critical point, but our results are model independent and include the effect of fluctuations.

\subsection{Comparison with Lattice Simulations}

Finite temperature lattice QCD simulations are ideally suited to testing many of the predictions made in this chapter. For a pedagogical introduction to and review of finite temperature lattice QCD simulations, see DeTar's article in this volume. The static correlation functions of the three dimensional theory are natural objects to consider in finite temperature (Euclidean) simulations. Also, it is much easier to vary parameters like the temperature and the bare quark mass in a lattice simulation than in a real experiment. Hence, it should be possible to measure the static critical exponents of section 2.1, and the equation of state and the scaling behaviour of the pion and sigma masses of section 2.2 on the lattice.

Present simulations 19, 20,21,22,23,24, 25, 26,27 provide evidence that in two flavour QCD the phase transition is second order, and that the order parameter is indeed $\mathcal{M}$. For example, in the results of Bernard et al 20 shown in Fig. 5, there are no signs of any discontinuities in the expectation value of the order parameter as a function of temperature. $\langle\bar{q} q\rangle$ decreases smoothly as a function of increasing temperature, as Fig. 1 leads us to expect for simulations done with nonzero quark masses if the transition is indeed second order for $m_{q}=0$. All these authors have looked unsuccessfully for signals of two phases coexisting at one temperature, which would be characteristic of a first order transition. These simulations are consistent with the transition being second order, but do not rule out the possibility that in better simulations with smaller quark masses a small discontinuity could be found, indicating that the transition is in fact weakly first order. Using the zero temperature $\rho$ mass to set the energy scale, Bernard et al.20.26 find that the temperature at which the decrease in the chiral order parameter occurs is about $140-160 \mathrm{MeV}$, significantly lower than in simulations without dynamical fermions. Over the same range of temperatures at which the chiral order parameter decreases, the Polyakov loop expectation value increases and the specific heat is large. Also, the calculations slow down - simulations must be run for a long time to get reliable results because large fluctuations occur. This is as would be expected near a second order critical point. Thus, in QCD with 2 dynamical quarks, it seems that the crossover associated with deconfinement, for which there is no order parameter, occurs at the same temperature as the chiral transition. The chiral transition is also a smooth crossover, but we expect it to approach a second 
Fig. 5. From Ref. [20]. The chiral order parameter in the lattice gauge theory simulations of Bernard et al. with two flavours of staggered quarks. The chiral order parameter is plotted as a function of $T$ for $m_{q}=0.025$ and $m_{q}=0.0125$ in lattice units. The simulations were done on a $12^{3} \times 6$ Euclidian lattice. On such a lattice, the period in the fourth direction is $1 / T$. Since the number of lattice sites in this direction (six) is not varied, to vary the temperature one varies the physical value of the lattice spacing. The fact that QCD is asymptotically free means that reducing the lattice coupling constant $g$ reduces the physical value of the lattice spacing and therefore corresponds to increasing the temperature. Hence, in the figure temperature increases to the right. The curve labeled $m_{q}=0$ is a linear extrapolation from the other two curves. We predict that if $m_{q}$ were lowered to zero, one would find the behaviour of Fig. 1 rather than a linear extrapolation.

order transition as $m_{q}$ is lowered further. All of this is in marked contrast to results of lattice gauge theory simulations with three or four species of light quarks, in which there is a first order chiral phase transition with latent heat, hysteresis, and abrupt changes in the values of observables.29,21,25,27 (Our results on the order of the chiral transition - second for two massless quarks and first for three or more - are also in agreement with results obtained by treating the QCD vacuum as an instanton liquid and making a mean-field approximation 54 )

Fig. 6. shows the behaviour of the screening mass (inverse correlation length) for the $\pi$ and $\sigma$ in the same simulation as Fig. 5. If the exponent $\eta$ were zero, then $m_{\pi}$ and $m_{\sigma}$ (which, recall, are susceptibilities ${ }^{-1 / 2}$ ) would have the same scaling behaviour as the screening masses of Fig. 6. Since $\eta$ is in fact small, the screening masses can be viewed as crude approximations to $m_{\pi}$ and $m_{\sigma}$. As we expect, the sigma screening mass decreases and the pion screening mass increases as the temperature is increased, and above $T_{c}$ the two masses each increase and appear to be becoming degenerate. This is evidence that the order parameter is indeed $\mathcal{M}$.

So far, we have discussed various qualitative lattice results, that are in agreement with our expectations. Before turning to recent progress on more quantitative tests, 
Fig. 6. From Ref. 20]. The inverse of the pion and sigma correlation lengths as a function of $T$ for $m_{q}=0.0125$ in lattice units in the same simulations as in Fig. 5. The screening masses are crude approximations to the inverse susceptibilities $m_{\pi}$ and $m_{\sigma}$ because $\eta$ is small. The qualitative behaviour should be compared to Figs. 2 and 3.

it is worth enumerating the logical possibilities which could lead to a failure of the hypothesis that a second order chiral phase transition with $O(4)$ exponents will be seen in lattice simulations. Perhaps the most likely alternative is that the transition could in fact be first order. As we will see in the next section, this occurs if the strange quark is too light. More generally, we only know that an infrared fixed point with appropriate symmetry exists. We do not know that QCD is in fact in the basin of attraction of this fixed point. A second logical possibility is that the Ginzburg region may be too small to see the true critical behaviour. Fluctuations of the order parameter are important only close to $T_{c}$ - for $t \equiv\left(T-T_{c}\right) / T_{c}<t_{G}$ where $t_{G}$, like $T_{c}$, is not universal. Outside the Ginzburg region, (i.e. for $t>t_{G}$,) mean field theory is valid. Thus, even if the chiral phase transition does have $O(4)$ exponents, these exponents can only be measured by simulations done for temperatures satisfying $t<t_{G}$. Simulations with larger $t$ would see the mean field exponents $\eta=0$ and $\nu=1 / 2 . t_{G}$, like $T_{c}$, cannot be predicted by arguments based on universality, and must be measured on the lattice. Can $t_{G}$ be very small? This is a logical possibility. For example, 55 in ordinary, low temperature, BCS superconductors, $t_{G} \sim\left(T_{c} / \varepsilon_{f}\right)^{4}$ where $\varepsilon_{f}$ is the Fermi energy. In these materials, $T_{c} / \varepsilon_{f} \sim 10^{-3}-10^{-4}$ and $t_{G}$ is minuscule. Recent numerical simulations by Kocic and Kogut 5 of the $2+1$ dimensional Gross-Neveu model in which mean field exponents are seen can be interpreted as a sign that $t_{G}$ is small in this theory. In QCD, there is no analogue of the small parameter $T_{c} / \varepsilon_{f}$, and so there is no reason to expect $t_{G}$ to be particularly small. Nevertheless, it remains a logical possibility. To exclude this scenario, it is therefore crucial to measure the 


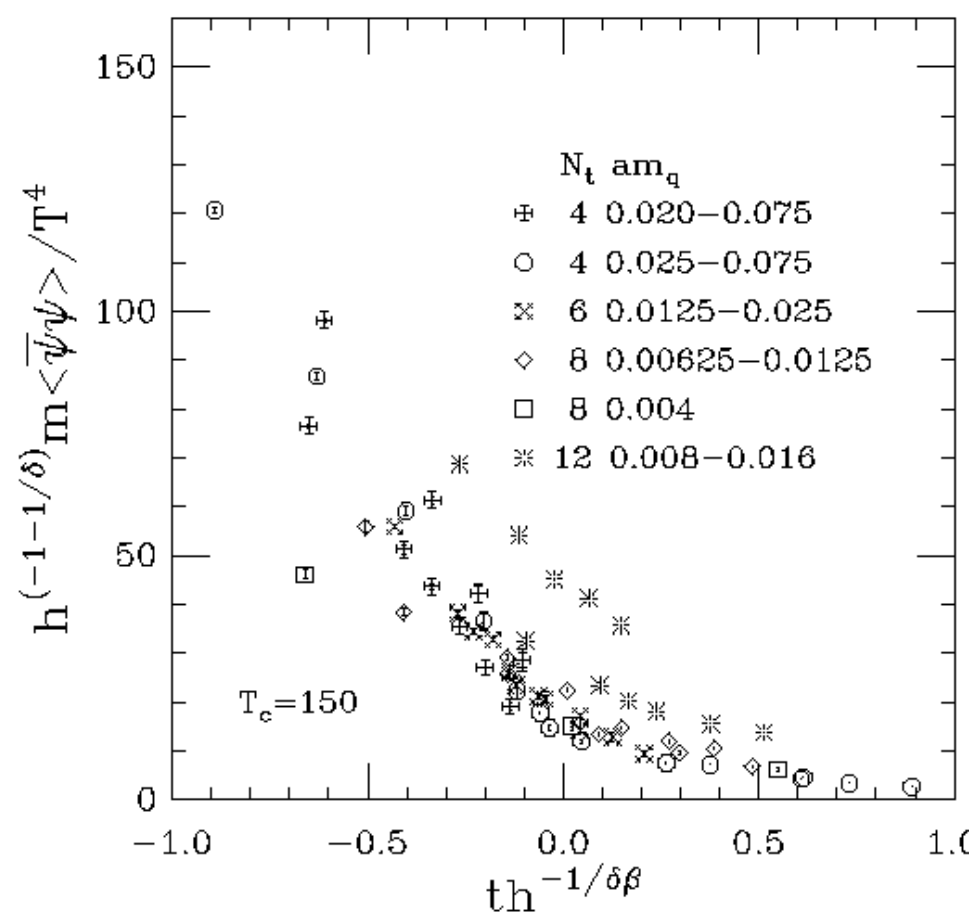

Fig. 7. From Ref. 57. DeTar's plot of $\tilde{y}$ vs. $\tilde{x}$ using $O(4)$ exponents.

critical exponents in QCD simulations with two flavours accurately enough to demonstrate that they deviate from their mean field values. The third logical possibility is that somewhere in theory space there is another infrared fixed point to which QCD is driven under renormalization. Were this the case, the chiral phase transition would be second order but would not have $O(4)$ exponents. Of the three logical possibilities, the third would be the most surprising.

Recently, a number of authors have begun to attempt quantitative comparisons between lattice simulations and expectations based on the hypothesis that the chiral transition is second order with $O(4)$ exponents. DeTar has pointed out 50 that it is possible to check that the order parameter $M$, the temperature $t$, and the symmetry breaking term $H \sim m_{q}$ are in fact related by a single equation of state (42). Following Binder, 58 DeTar writes (42) in a different, but equivalent, fashion. Define

$$
\tilde{x} \equiv t / H^{1 / \beta \delta}, \quad \tilde{y} \equiv M / H^{1 / \delta} .
$$

Then, the equation of state (42) is equivalent to

$$
\tilde{y}=g(\tilde{x})
$$

where $g(\tilde{x})$ is a universal function related to $f(x)$ of (42) by

$$
\tilde{x}=g^{-1}(\tilde{y})=x^{-1 / \beta \delta} f^{-1}(x) .
$$




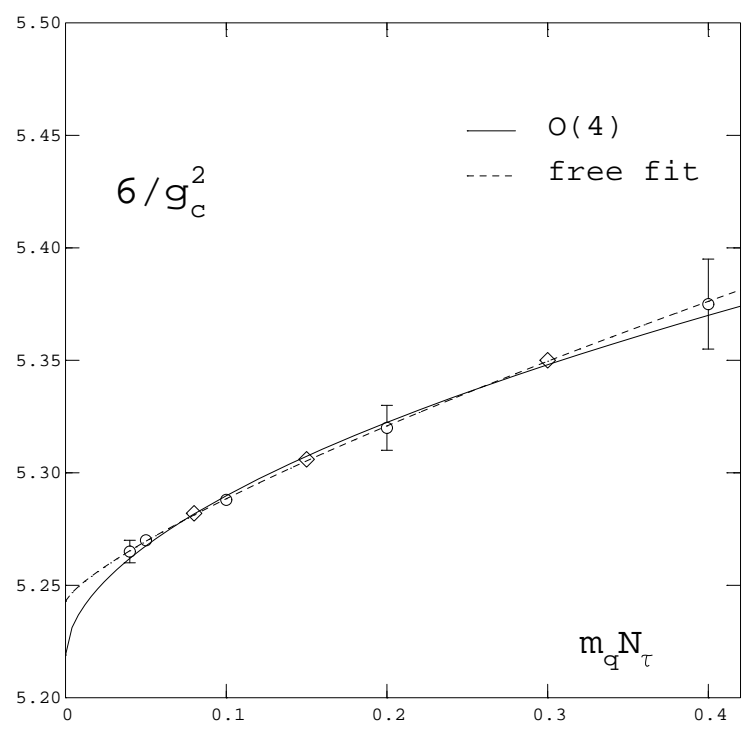

Fig. 8. From Ref. [60]. Pseudocritical coupling as a function of quark mass for simulations done with $N_{\tau}=4$ lattice sites in the Euclidean time direction. The dashed curve is a three parameter fit to (59). The solid curve is a two parameter fit with $1 / \beta \delta$ set to its $O(4)$ value.

Fig. 7 is DeTar's plot of $\tilde{y}$ versus $\tilde{x}$ for a number of simulations2023,24,26, 60 with various quark masses, temperatures, and lattice sizes. If (56) is correct, all the points should lie on a single curve. In producing the figure, DeTar has used the $O(4)$ values for $\beta$ and $\delta$, and has adjusted $T_{c}$ to $150 \mathrm{MeV}$ to obtain the best agreement, although he notes that $160 \mathrm{MeV}$ works comparably well. DeTar's plot is encouraging, but not yet quantitative. For one thing, the preliminary data on the largest lattices with 12 lattice sites in the $t$ direction are not in good agreement with the other data, suggesting that finite size effects may be important. In the future, with better simulations, it will be possible to apply this analysis more quantitatively - the simulation results will be fit to (56) yielding best fit values for $T_{c}, \beta$ and $\delta$, along with error estimates.

Karsch 59 has pioneered another way of measuring the critical exponents in a lattice simulation. From Fig. 3. we see that for $H \neq 0, m_{\sigma}$ has a minimum at $t=t_{\mathrm{pc}}>0$, which Karsch calls the pseudocritical temperature. Because $t, H$, and $M$ are related by (42) or equivalently (56),

$$
t_{\mathrm{pc}} \sim H^{1 / \beta \delta} .
$$

Karsch therefore notes that in lattice simulations, the pseudocritical coupling $g_{\mathrm{pc}}$ at which the $\sigma$ susceptibility peaks should depend on $m_{q}$ according to

$$
\frac{6}{g_{\mathrm{pc}}^{2}\left(m_{q}\right)}=c_{0}+c_{1} m_{q}^{1 / \beta \delta},
$$

where $c_{0}$ is related to $T_{c}$ for the second order transition with massless quarks, and neither $c_{0}$ nor $c_{1}$ are universal. Karsch and Laermann's plot 60 of the pseudocritical 


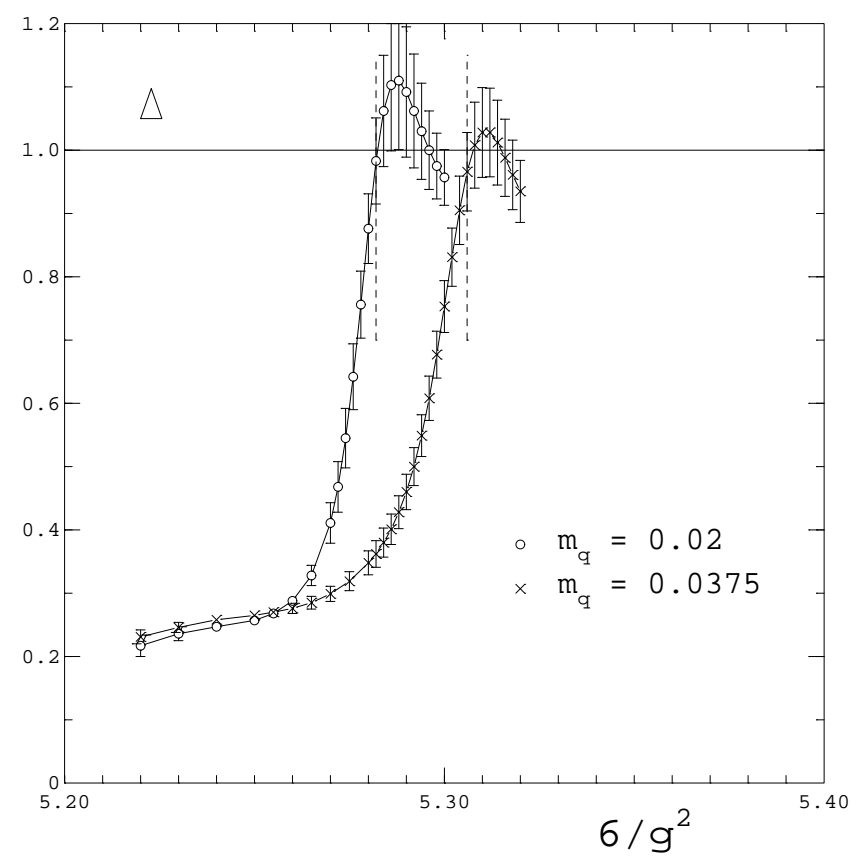

Fig. 9. From Ref. [60]. The ratio of susceptibilities $\Delta \equiv m_{\pi}^{2} / m_{\sigma}^{2}$ as a function of temperature for two different quark masses.

coupling in various simulations $1922,22,60$ is shown in Fig. 8. Fitting the three parameters $c_{0}, c_{1}$, and $1 / \beta \delta$ yields $1 / \beta \delta=0.77 \pm 0.14$, which is slightly higher than the $O(4)$ value $1 / \beta \delta=0.55 \pm .02$ and which is in agreement with the mean field value $1 / \beta \delta=2 / 3$. However, fitting $c_{0}$ and $c_{1}$ with $1 / \beta \delta$ fixed to its $O(4)$ value yields just as good a fit. Simple inspection of the figure leads to the conclusion that it is too early to extract the value of the exponent convincingly — simulations with lighter quarks are needed.

Karsch and Laermann 60 have evaluated both the $\pi$ and the $\sigma$ susceptibilities. From these, they extract a direct measurement of the exponent $\delta$. From (45) and (46), we see that the quantity $m_{\pi}^{2} / m_{\sigma}^{2}$ which Karsch and Laermann call $\Delta$ is given by

$$
\frac{1}{\Delta}=\frac{m_{\sigma}^{2}}{m_{\pi}^{2}}=\delta-\frac{x f^{\prime}(x)}{\beta f(x)}
$$

For $t>0, \Delta \rightarrow 1$ for $m_{q} \rightarrow 0$ and for $t<0, \Delta \rightarrow 0$ for $m_{q} \rightarrow 0$. Most important, however, is that for any quark mass, $\Delta=1 / \delta$ at $t=x=0-i$.e. at $T=T_{c}$. Curves of $\Delta$ as a function of $6 / g^{2}$ for different quark masses should cross at $6 / g_{c}^{2}$, and at that coupling they should have $\Delta=1 / \delta$. Karsch and Laermann's results are shown in Fig. 9. They find

$$
0.21<1 / \delta<0.26
$$

This is in agreement with the $O(4)$ value $1 / \delta=0.208 \pm .002$ and disagrees with the mean field value $1 / \delta=1 / 3$. To make this result even more compelling, it would be 
good to have curves for lighter quarks to see that they all cross at the same $T_{c}$ and $\Delta$, and to see $\Delta$ decreasing toward zero as $m_{q} \rightarrow 0$ for $T<T_{c}$.

One critical exponent which has not been mentioned in this section is $\alpha$, which is associated with the specific heat. Because $\alpha<0$, there should be a cusp in the specific heat. However, the specific heat can also have a large analytic contribution. In fact, as the temperature is raised through the chiral transition, deconfinement also occurs and the number of degrees of freedom in thermal equilibrium increases substantially, leading to a large specific heat 61 This large contribution to the specific heat is an analytic function of $T$ even for $m_{q}=0$, while the contribution from the chiral order parameter is not. Nevertheless, because the smooth part of the specific heat is so large, extracting the non-analytic part and thus $\alpha$ will be difficult. 62

By this point it should be clear that quantitative tests of the hypothesis that the QCD phase transition is in the same universality class as the $O(4)$ magnet are just beginning to be possible. To date, lattice simulations of two flavour QCD are in qualitative agreement with this hypothesis. As lattice simulations improve, these tests will become more and more quantitative. It should soon be possible to exclude conclusively the possibility that the exponents take on mean field values. Indeed, the results of Karsch and Laermann for $1 / \delta$ are close to doing this already. This would demonstrate that the simulations are close enough in temperature to $T_{c}$ that the fluctuations of the order parameter are important - i.e. the simulations are within the Ginzburg region. This would be reassuring, as it would imply that as simulations improve further the true critical behaviour will be explored, and the hypothesis that the transition is in the $O(4)$ universality class will be tested quantitatively.

Now, some caveats. Present lattices are small. Ideally, one wants the correlation length to be large compared to the lattice spacing and small compared to the lattice size, and this requires much larger lattices. (Berera 63 has suggested, however, that this problem can be turned into a virtue - finite size effects and finite lattice spacing effects affect the critical behaviour and therefore can be used to measure critical exponents like $\nu$.) However, the fundamental reason why present simulations do not offer more quantitative tests of the predictions in this chapter is that to date quark masses have been so large that correlation lengths do not get very long at $T_{c}$. For example, in the work of Bernard et al. 20 the correlation length in the pion channel at $T_{c}$ is only about 2.5 lattice lengths. To really probe the critical phenomena, lighter quarks are needed. Unfortunately, longer correlation lengths are necessarily accompanied by numerical critical slowing, and this makes simulations challenging.

Recent work by the Columbia group 64 offers another indication that lighter quark masses are necessary in order to quantitatively probe the critical phenomena. They vary the "valence quark mass" (the quark mass in the $\bar{q} q$ operator inserted in order to measure the order parameter) and the "sea quark mass" (the mass of the dynamical quarks in the fermion determinant) independently. $m_{\text {sea }}$ cannot be made too small because to do so would slow the simulation down; $m_{\text {val }}$ can be made arbitrarily small. 
The first result they find is that the order parameter goes to zero as a temperature dependent power of $m_{\mathrm{val}}$ for fixed $m_{\text {sea }}=0.01$. (All masses are in lattice units.) They interpret the power law behaviour they see for $m_{\text {val }}$ between about $10^{-2}$ and $10^{-4}$ as a sign of some sort of critical behaviour, but no interpretation in terms of the exponents we have discussed is possible, since these exponents are only relevant for the physical case where $m_{\mathrm{val}}=m_{\text {sea }}=m_{q}$. They also find indications that for fixed $m_{\mathrm{val}}$, variations of $m_{\text {sea }}$ which are accessible with their present simulations (i.e. down to $m_{\text {sea }}=0.01$ ) seem to renormalize $6 / g^{2}$ and have no other effect. (It is known 65 that the effect of heavy quarks is to shift $6 / \mathrm{g}^{2}$.) If these preliminary results are confirmed, they suggest that $m_{\text {sea }}$ must be reduced further than has been possible to date in order to see the critical phenomena characteristic of $m_{q} \rightarrow 0$ discussed in this article.

Another hurdle to be overcome before lattice simulations can measure the critical properties of the QCD phase transition is that any lattice implementation of fermion fields only exhibits the full chiral symmetry in the continuum limit. Thermodynamic simulations with Wilson fermions are difficult because Wilson fermions break chiral symmetry completely. Larger lattices or an improved action are required to avoid lattice artifacts 57.66 If staggered fermions are used, one starts with four flavours of fermions which in the continuum have an $S U(4) \times S U(4)$ chiral symmetry. On the lattice, a $U(1) \times U(1)$ subgroup is all that remains. In order to study two flavours of fermions, one takes the square root of the fermion determinant in the lattice action. It is not at all clear what this does to the lattice chiral symmetries. What is known is that all three pions become light only in the continuum limit. Finite lattice spacing effects leave one pion light, while making two of them heavy. A transition in which a $U(1) \times U(1)$ symmetry breaks to $U(1)$ would be in the universality class of the $N=2$ magnet, as Boyd et al.67 have discussed. It is therefore possible that $O(2)$ exponents will be measured at first, and the $O(4)$ exponents will be seen only as finer lattices become possible and the continuum limit is approached. Therefore, it would be nice to be able to distinguish $O(4)$ exponents from $O(2)$ exponents, but this will require much greater accuracy than is presently possible. (For example, $\delta=4.808 \pm .007$ and $1 / \beta \delta=0.60 \pm .01$ at an $O(2)$ transition, and whereas distinguishing $O(4)$ exponents from mean field exponents seems feasible in the short term, distinguishing between $O(4)$ and $O(2)$ will be more difficult.) Therefore, for staggered as for Wilson fermions, before we are able to make confident quantitative tests of our predictions for the critical phenomena we need finer lattices so that extrapolation to the continuum limit can be done. Eventually, the results for staggered and Wilson fermions must agree when so extrapolated.

At present, it is clear, many caveats remain to be dealt with before quantitative measurements of the critical exponents in lattice simulations become compelling. The situation will improve steadily as simulations become possible on larger lattices with smaller quark masses. In the future, simulations which have longer correlation lengths and which are closer to the continuum limit will be able to measure critical exponents, 
correlation functions, susceptibilities, and the critical equation of state, and test the hypothesis that the chiral phase transition in QCD with two flavours of quarks is in the same universality class as the $O(4)$ magnet. At present, this hypothesis has passed a number of qualitative tests.

\subsection{The Influence of the Strange Quark}

To this point in this chapter, we have described a world with two massless or light quarks, and hence we have implicitly been taking the strange quark mass to be infinite. Pisarski and Wilczek showedes that if the up, down, and strange quarks are all massless, then the chiral phase transition is first order. With three massless quarks, the order parameter $\mathcal{M}_{j}^{i}$ is a $3 \times 3$ matrix and there are two possible quartic couplings: $\lambda_{1} \operatorname{tr}\left(\mathcal{M}^{\dagger} \mathcal{M}\right)^{2}$ and $\lambda_{2}\left(\operatorname{tr} \mathcal{M}^{\dagger} \mathcal{M}\right)^{2}$. The renormalization group equations for this model have been studied to lowest order in $\epsilon$ by Pisarski and Wilczek 28 and Paterson 68 There is indeed a fixed point, but it is not stable. For three or more flavours of massless quarks, there are always directions in $\lambda_{1}-\lambda_{2}$ space around the fixed point for which renormalization toward the infrared drives the theory away from the fixed point. Assuming the $O(\epsilon)$ result that there is no infrared stable fixed point is correct, the phase transition must be first order. This result has been verified by Gausterer and Sanielevici.69 They did numerical simulations of the $3 \times 3$ matrix model and found that the transition is indeed first order.

The chiral phase transition is second order in a world with two massless quarks and first order in a world with three. Hence, as the strange quark mass is reduced from infinity to zero, at some point the phase transition must change from second order to first order. This point is called a tricritical point. In a lattice simulation, the strange quark mass could be tuned to just the right value to reach the tricritical point. We now discuss the critical exponents that would be observed in such a simulation.

Let us consider the effect of adding a massive but not infinitely massive strange quark to the two flavour theory. This will not introduce any new fields which become massless at $T_{c}$, and so the arguments leading to the free energy (27) are still valid. The only effect of the strange quark, then, is to renormalize the couplings. Renormalizing $\mu^{2}$ simply shifts $T_{c}$, as does renormalizing $\lambda$ unless $\lambda$ becomes negative. In that case, one can no longer truncate the Landau-Ginzburg free energy at fourth order. After adding a sixth order term, the free energy becomes

$$
F=\int d^{3} x\left\{\frac{1}{2}(\nabla \phi)^{2}+\mu^{2} \phi^{2}+\lambda\left(\phi^{2}\right)^{2}+\kappa\left(\phi^{2}\right)^{3}-H \sigma\right\} .
$$

While for positive $\lambda, \phi^{2}$ increases continuously from zero as $\mu^{2}$ goes through zero, for negative $\lambda, \phi^{2}$ jumps discontinuously from zero to $|\lambda| /(2 \kappa)$ when $\mu^{2}$ goes through $\lambda^{2} /(4 \kappa)$. Hence, the phase transition has become first order. Thus, at some $m_{s} \equiv m_{s}^{*}$ at which $\lambda=0$ at $T=T_{c}$, the phase transition changes continuously from second order to first order. 
The nonanalytic behaviour of thermodynamic functions near tricritical points, just as near ordinary critical points, is universal. Hence, it is natural to propose that QCD with two massless flavours of quarks and with $T$ near $T_{c}$ and $m_{s}$ near its tricritical value $m_{s}^{*}$ is in the universality class of the $\phi^{6}$ Landau-Ginzburg model (62). This model has been studied extensively. 0 Because the $\phi^{6}$ interaction is strictly renormalizable in three dimensions, this model is much simpler to analyze than the $\phi^{4}$ model of the ordinary critical point. No $\epsilon$ expansion is necessary, and the critical exponents all take their mean field values. There are calculable logarithmic corrections to the scaling behaviour of thermodynamic functions, 70 but we will limit ourselves here to determining the mean field tricritical exponents.

In mean field theory, the correlation function in momentum space is simply $G_{\alpha \beta}(k)=\delta_{\alpha \beta}\left(k^{2}+\mu^{2}\right)^{-1}$. Since $\mu^{2} \sim t$, this gives the exponents $\eta=0, \gamma=1$ and $\nu=1 / 2$. To calculate $\alpha$ and $\beta$, we minimize $F$ for $H=\lambda=\nabla \phi=0$, and find $\alpha=1 / 2$ and $\beta=1 / 4$. To calculate $\delta$, we minimize $F$ for $t=\lambda=\nabla \phi=0$ and find $\delta=5$.

The result for the specific heat exponent $\alpha$ is particularly interesting, since it means that the specific heat diverges at the tricritical point, unlike at the ordinary critical point. This means that whereas for $m_{s}$ large enough that the transition is second order the specific heat $C(T)$ has a cusp but is finite at $T=T_{c}$, as $m_{s}$ is lowered to the tricritical value $C\left(T_{c}\right)$ should increase since at the tricritical point it diverges. This behaviour should be seen in future lattice simulations.

At a tricritical point there is one more relevant operator than at a critical point, since two physical quantities $\left(t\right.$ and $m_{s}$ ) must be tuned to reach a tricritical point. Hence, a new exponent $\phi_{t}$, the crossover exponent, is required. For $\lambda \neq 0$, tricritical behaviour will be seen only for $|t|>t^{*}$, while for $|t|<t^{*}$, either ordinary critical behaviour or first order behaviour (depending on the sign of $\lambda$ ) results. $t^{*}$ depends on $\lambda$ according to

$$
t^{*} \sim \lambda^{1 / \phi_{t}}
$$

The mean field value of $\phi_{t}$ is obtained by minimizing the free energy $F$ for $H=\nabla \phi=$ 0 , and is $\phi_{t}=1 / 2$. These mean field tricritical exponents, $\alpha=1 / 2, \beta=1 / 4, \gamma=1$, $\delta=5, \eta=0, \nu=1 / 2$, and $\phi_{t}=1 / 2$ describe the real world if $m_{s}$ is close enough to the tricritical value, and will describe future lattice simulations with $m_{s}$ chosen appropriately.

We now construct a phase diagram showing the order of the chiral transition as a function of $m_{s}$ and the light quark mass $m_{q}$. In the $\left(m_{q}, m_{s}\right)$ plane, there is a region around $(\infty, \infty)$ in which all quarks are sufficiently massive that a first order deconfinement transition is obtained. In a region around $(0,0)$, all quarks are light enough that the first order chiral transition characteristic of the theory with three massless quarks is obtained. On the left side of Fig. $10, m_{q}=0$ and the chiral phase transition is first order for $m_{s}<m_{s}^{*}$ and second order for $m_{s}>m_{s}^{*}$. For $m_{q} \neq 0$, and $m_{s}$ large enough, the chiral transition is a smooth crossover. For $m_{s}>m_{s}^{*}$ 


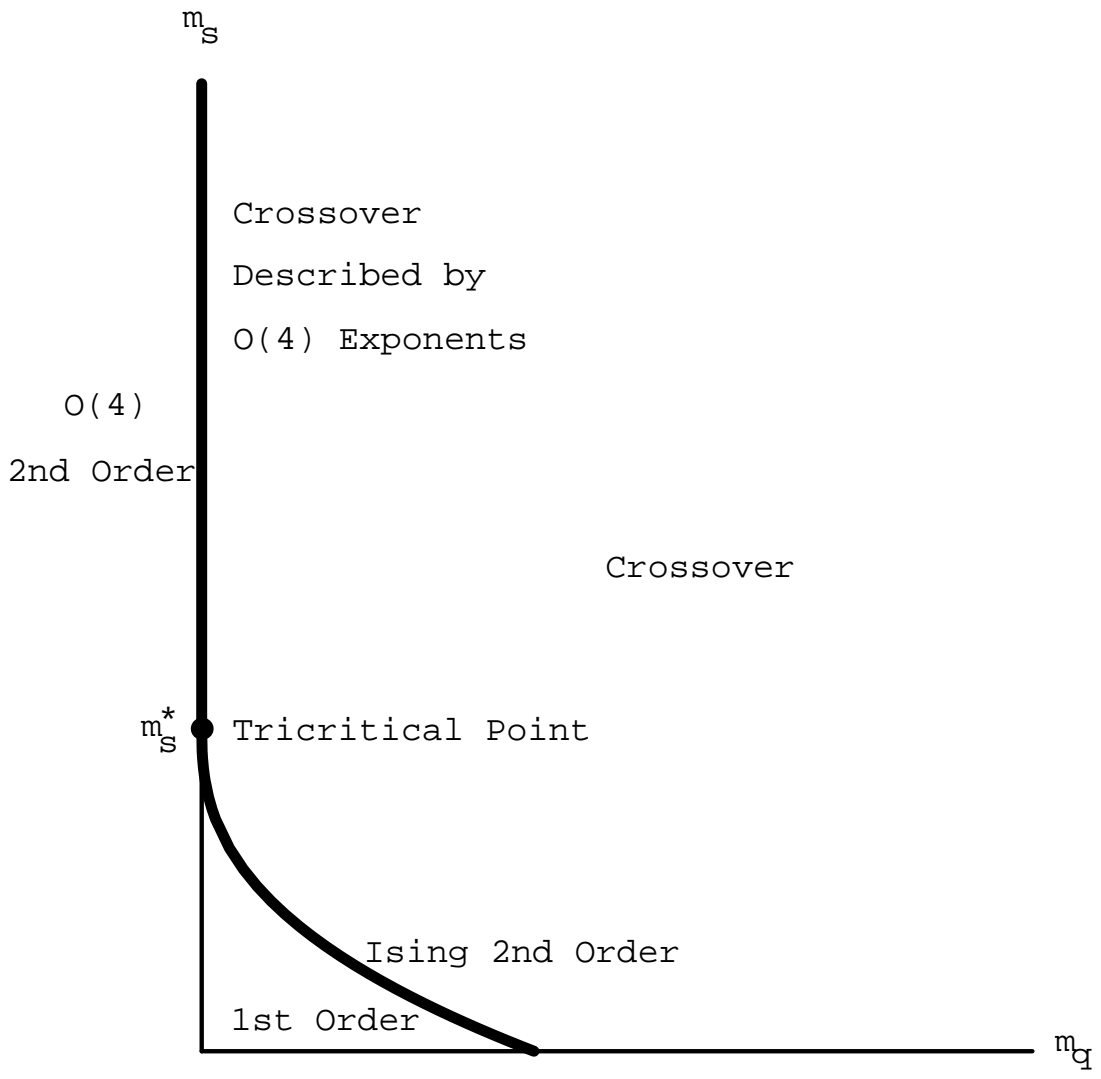

Fig. 10. A phase diagram showing how the order of the chiral transition depends on $m_{q}$ and $m_{s}$. For large $m_{s}$, there is a second order transition with $O(4)$ exponents at $m_{q}=0$. For $m_{q}$ nonzero, there is a smooth crossover which, for small $m_{q}$, looks like one of the curves of Fig. 1. For $m_{q}=0$, the transition becomes first order when $m_{s}$ is reduced below its tricritical value $m_{s}^{*}$. In a region around $m_{s}=m_{q}=0$, the transition is first order. The boundary of this region is a curve of second order transitions with Ising model exponents. 1 In the figure, this curve is drawn with the shape $\left(m_{s}^{*}-m_{s}\right) \sim m_{q}^{2 / 5}$, which is valid near the tricritical point.

and $m_{q}$ nonzero but small enough, the crossover is described by $O(4)$ exponents, as discussed in previous sections. At the boundary between the first order region and the smooth crossover region, the transition has been analyzed in mean field theory by Gavin, Gocksch and Pisarski 71 They find that along this curve in the $\left(m_{q}, m_{s}\right)$ plane, a single scalar field is massless and therefore conjecture that along this curve the transition is second order with Ising critical exponents. (The scalar which is massless is pure $s \bar{s}$ near $m_{s}=0$, becomes an $S U(3)$ singlet as one follows the curve to the point at which $m_{s}=m_{q}$ and becomes the $\sigma$ of previous sections as one follows the curve further toward the tricritical point at which $m_{q}=0$.) Using (62), we can deduce the shape of the curve of second order transitions near the tricritical point. For $\lambda<0$, consider turning on a small $H$. $H$ breaks the $O(4)$ symmetry, and 
makes the order parameter point in the $\sigma$ direction. Working to lowest order in $H$, we find that $\sigma$ jumps discontinuously between degenerate minima at $\sigma=2 H \kappa / \lambda^{2}$ and $\sigma=\sqrt{|\lambda| /(2 \kappa)}-(1 / 2) H \kappa / \lambda^{2}$ at the temperature at which $\mu^{2}$ goes through $\lambda^{2} /(4 \kappa)+H \sqrt{2 \kappa /|\lambda|}$. As $H$ increases, the size of the jump in $\sigma$ at $T_{c}$ shrinks, until at some $H \sim|\lambda|^{5 / 2} / \kappa^{3 / 2}$ the two degenerate minima merge and the transition is second order. For even larger $H$, the transition is a smooth crossover. Since $H \sim m_{q}$ and $\lambda \sim\left(m_{s}-m_{s}^{*}\right)$, we conclude that near the tricritical point, the curve of second order transitions separating the region in which the transition is first order from the region in which it is a smooth crossover has the shape

$$
\left(m_{s}^{*}-m_{s}\right) \sim m_{q}^{2 / 5}
$$

Away from the tricritical point, a curve of second order transitions exists, 11 but nothing is known about its shape.

The real world is one point on Fig. 10. Lattice simulations, on the other hand, can be done with varying quark masses. Thus, on the lattice unlike in the real world it will be possible to test and explore all the physics of Fig. 10. It is clearly very important to know where the physical values of $m_{s}$ and $m_{q}$ lie in Fig. 10. This is not a universal question, and therefore can only be answered by experiment or by lattice simulations.

In simulations done on a $16^{3} \times 4$ lattice with staggered fermions, 21 the Columbia group found a first order transition for $m_{s}=m_{q}=0.025$ in lattice units, and found no sign of first order behaviour in simulations with only two flavours of fermions and in a simulation with $m_{s}=0.1$ and $m_{q}=0.025$. By measuring zero temperature hadron masses, they estimate that in the latter simulation $m_{q}$ is larger than its physical value and, most important, $m_{s}$ is smaller than its physical value. These results suggest that in this simulation, $m_{s}$ is greater than $m_{s}^{*}$, and furthermore that the physical value of $m_{s}$ is even larger. Thus, the Columbia results suggest that the real world is at a point in Fig. 10 somewhere in the region labelled "crossover described by $\mathrm{O}(4)$ exponents."

Recently, Iwasaki et al. 22 have announced results from simulations with two light and one heavy Wilson fermions. In agreement with the Columbia results, they find a first order transition when all three quarks are light, and no sign of a first order transition in simulations with two light quarks. However, they report seeing a two state signal characteristic of a first order transition in simulations with two light quarks and a strange quark either of mass $m_{s} \sim 150 \mathrm{MeV}$ or of mass $m_{s} \sim 400 \mathrm{MeV}$. This suggests that the real world lies in the region of Fig. 10 labelled "first order", and is inconsistent with the Columbia results. There are a number of extensions to the work of Iwasaki et al. that would make it more compelling. First, in order to demonstrate consistency with the result (from their own simulations and those of others) that the transition is a smooth crossover for two flavours, it would be good if a simulation was performed with a heavy enough strange quark that no signs of a first order transition are seen. Second, in order to more confidently make comparisons 
with the Columbia results, a measurement of the zero temperature hadron masses would be helpful. Finally, as discussed briefly in the previous section and at length in DeTar's article in this volume, studying the chiral transition with Wilson fermions is tricky because of potential lattice artifacts since the chiral symmetry is completely broken on the lattice. At the end of the day, however, when simulations are done on large enough lattices, it should not matter whether staggered or Wilson fermions are used. Although the results of Ref. [21] are at present more convincing than those of Ref. [72], the question of where on Fig. 10 the real world lies remains open. Time (i.e. further simulations) will tell.

\subsection{Implications}

We have seen in the previous two sections that the physics of the chiral phase transition in QCD is well-suited for study on the lattice. The hypothesis that the chiral transition in two flavour QCD is second order with $O(4)$ critical behaviour has many implications which are being and will be tested in lattice simulations. The exploration of the $\left(m_{q}, m_{s}\right)$ plane has also begun. Let us now turn to real experiments, as opposed to those on the lattice. Here, we are not free to dial the bare quark masses. We will see that this is unfortunate, particularly in our discussion of heavy ion collisions. First, however, let us dispose of two other possible arenas for testing our results.

The QCD phase transition certainly occurred in the early universe. Indeed, much work has been done on possible observable cosmological effects of this transition, if it is first order. There have been speculations 73 that a strongly first order transition could have affected big bang nucleosynthesis and therefore left a signature in the primordial nuclear abundances, ${ }^{b}$ or could have left an imprint in the form of gravitational waves, or could even have left relic chunks of new forms of matter. Unfortunately, we have seen that for physical values of the strange quark mass, it is likely but not certain that the transition is second order. In fact, because the up and down quarks are not massless, the second order transition is smoothed into a crossover. We can think of no observable consequences of a second order QCD phase transition in the early universe, and even fewer consequences of a smooth crossover.

There is one possibly useful cosmological (non)consequence of a QCD transition which is not first order. In the early universe, the expansion rate during the transition is very slow compared to QCD time scales, and thermal equilibrium is maintained. Therefore, if the transition is not first order there is no entropy production at the

\footnotetext{
${ }^{b} \mathrm{~A}$ strong first order phase transition creates inhomogeneities in the baryon number density of the universe which later affect nucleosynthesis. .3 Present observations of nuclear abundances actually constrain big bang nucleosynthesis tightly enough that they disallow models in which the abundances are significantly affected by inhomogeneities in the baryon number density. 54 These cosmological observations, therefore, are evidence that the QCD phase transition is not strongly first order, in agreement with our results.
} 
transition. Thus, whatever mechanism generates the baryon number density of the universe at some higher energy scale need only generate the present baryon to entropy ratio, and need not generate a larger baryon number to compensate for dilution at a first order QCD phase transition.

Now, let us turn from cosmology to laboratory experiments. Since certain antiferromagnets including dysprosium have order parameters in the $N=4$ universality class, 75 experiments on the phase transition in these materials could help us understand the QCD transition. $\$$ Alas, in these magnets there is a quartic operator like $\left(\pi_{1}^{2}+\pi_{2}^{2}\right)\left(\pi_{3}^{2}+\sigma^{2}\right)$ which is allowed by the microscopic hamiltonian of the magnets,, 6 but not by that of QCD. This operator makes the symmetric Heisenberg fixed point infrared unstable, and either makes the phase transition first order or makes it second order but governed by an anisotropic fixed point. Hence, dysprosium is not a suitable arena for learning about the QCD phase transition.

Finally, we turn to relativistic heavy ion collisions. As we discussed in the Introduction, in a sufficiently high energy collision the baryon number of the incident incident nuclei ends up in that part of the plasma heading approximately down the beam pipes, and the central rapidity region consists of a hot plasma with approximately zero baryon number. In the remainder of this article, we will study the behaviour of the plasma in the central rapidity region as it expands and cools through $T=T_{c}$ and eventually hadronizes and becomes pions which fly off and are detected.

The defining characteristic of a second order phase transition is the divergence of correlation lengths. How could this feature be observed here? Large volumes of space with the order parameter correlated and pointing in a direction different than the true vacuum (i.e. sigma) direction will become regions in which the order parameter oscillates coherently about the sigma direction. After hadronization, correlated volumes will turn into regions of space where the ratio of the number of charged pions to neutral pions has some fixed value. In the standard scenario 31 described in the Introduction, the longitudinal expansion is boost invariant and different positions in the plasma along the beam direction become different rapidities as the plasma expands. Therefore, one would hope that a signal of a second order phase transition would be fluctuations in the ratio of charged to neutral pions as a function of rapidity. Such phenomena will be our subject in the next chapter.

A prerequisite for the fluctuations discussed above to be observable is that the correlation length must get long compared, say, to $T_{c}$. To determine whether this does indeed happen, we must leave our universality safety net behind, since neither $T_{c}$ nor the magnitudes of correlation lengths are universal. From Fig 2, it is immediately apparent that we have a problem. The longest correlation length is in the pion channel, and the pion mass is increasing with temperature. This suggests that the pion mass at $T_{c}$ is larger than $m_{\pi}(T=0)=135 \mathrm{MeV}$. This result can be checked by comparing with (non-universal) results obtained in a variety of models. In chiral perturbation theory using the nonlinear sigma model, 77 in the linear sigma 
model, 8 and also in the Nambu-Jona-Lasinio model, 3 the pion mass increases from its zero temperature value as the temperature is increased from zero. Working in a specific four-dimensional model forces one to make a mean field 53 or one-loop 89 or low temperature 77 approximation which is not valid near $T=T_{c}$. This precludes obtaining good results for universal quantities, but allows rough estimation of non-universal quantities like $T_{c}$ and the low temperature behaviour of the pion mass. Non-universal analyses suggest that $m_{\pi}$ increases with temperature as $T$ increases from zero, and our universal result shows that it is increasing with temperature for $T \sim T_{c}$. Hence, it seems clear that the longest correlation length at $T_{c}$ will be shorter than $(135 \mathrm{MeV})^{-1}$. This is to be compared to $T_{c}$ itself, which for the case of two massless quarks is around 140-160 MeV 2026 Hence, even though the quark masses are indeed small $(\sim 10 \mathrm{MeV})$, the magnetic field $H$ proportional to $m_{q}$ is large enough to prevent any correlation lengths from reaching interesting values.

There is an appropriate quantitative criterion to determine whether an equilibrium second order phase transition leads to dramatic effects. One compares the energy in a correlation volume just below $T_{c}$ with the zero temperature pion mass to determine whether or not the correlated volume can become a large number of pions. Using the lattice simulations of Ref. [20], we can make a crude attempt at this comparison. The sum of the energy and pressure in a correlation volume is about $1 / 4$ the zero temperature $\rho$ mass. Taken literally, this means that each correlation volume becomes only one or two pions in the detector. In these simulations, $m_{q}$ is somewhat larger than its physical value, perhaps by a factor of two, and there are many other caveats as we have seen, so this estimate should not be taken literally. Nevertheless, it seems clear that the physical value of $m_{q}$ is large enough that in an equilibrium phase transition a correlation volume at $T_{c}$ does not evolve into a large number of correlated zero temperature pions. This is not encouraging.

We pause here for an aside. The reader may be wondering why, when the seemingly small equal quark mass $m_{u}=m_{d}=m_{q}$ has such deleterious effects, we have completely neglected the difference between the up and down quark masses. Unequal quark masses allow terms of the type 10

$$
\Delta F \propto(\delta m)^{2}\left(\sigma^{2}-\pi_{3}^{2}+\pi_{1}^{2}+\pi_{2}^{2}\right)
$$

If one is close enough to the critical point that this term matters, one will discover an anisotropic fixed point rather than the symmetric Heisenberg fixed point. However, while the effect of a common quark mass, namely the mass of the pion, is comparable to $T_{c}$, the effect of (65) is much smaller. For example, the QCD contribution to the difference in mass between the charged and neutral kaons is about $5 \mathrm{MeV} .79$ Therefore, we need not worry about (65) in real experiments since it is much less important than the effect of the "magnetic field" proportional to the common up and down quark mass. Of course, (65) could be introduced and studied on the lattice.

It seems likely that if the standard scenario for heavy ion collisions in which 
the QCD plasma cools through $T_{c}$ while staying close to thermal equilibrium is correct, then no correlation lengths will get long enough for there to be any dramatic observable effects of the phase transition. The chiral "transition", like the confinement/deconfinement "transition," will be a smooth crossover. If we were able to dial down the quark masses and hence the pion mass, phenomena associated with a second order transition would become more prominent. Alas, in the real world, unlike on a lattice, we have no such freedom. Let us hope that we will reach less disappointing conclusions in the next chapter when we consider the physics of the QCD phase transition in a heavy ion collisions in which the chiral order parameter does not stay in thermal equilibrium.

The future of the study of the chiral phase transition in QCD on the lattice looks promising. As simulations improve, investigations of the plethora of static critical phenomena we discussed in the first sections of this chapter will become better and better. Critical exponents, the equation of state, the critical behaviour of the pion and sigma susceptibilities, and tricritical exponents, are all out there waiting to be measured. The hypothesis that QCD with two flavours of massless quarks has a second order chiral phase transition in the same universality class as the $O(4)$ Heisenberg magnet will be tested more and more quantitatively.

\section{From Disorder to Long Wavelength Oscillations: The QCD Phase Tran- sition Far From Thermal Equilibrium}

The gloomy penultimate paragraph of the previous chapter began with a conditional sentence. In this chapter we will consider the observable effects in heavy ion collisions if the plasma does not stay close to thermal equilibrium through the transition. There are tantalizing hints in cosmic ray physics that point in this direction.

Among the zoo of high energy cosmic ray events which have been detected in emulsion experiments are a particularly peculiar class of events called Centauro $\$ 80$ discovered in emulsion experiments at the Mount Chacaltaya Observatory. Centauros are events with total energy of order $1000 \mathrm{TeV}$ in which many (of order 100) charged hadrons each with energies of a several $\mathrm{TeV}$ and very few photons or electrons are seen in a cosmic ray induced shower. In a normal event, the charged hadrons are accompanied by photons from the decay of neutral pions. Centauros have also been seen in emulsions exposed at the Pamir Observatory and the Mount Fuji Observatory. experimental situation is murky, however, because more recent searches at Mts. Kanbala and Fuji 82 failed to find any Centauros. 33 Events called mini-Centauros which have fewer charged hadrons than Centauros but which also have very few photons or electrons have also been observed. In the sample of events in described by Lattes et al. 80 there were 5 Centauros and 13 mini-Centauros, representing about $1 \%$ and $2 \%$ of the events seen with energy of the appropriate order of magnitude. Cosmic ray

shower simulations do predict events which can be interpreted as mini-Centauros, 84 
but it is difficult to interpret Centauros normally. 84

The JACEE collaboration has flown balloon borne emulsion detectors to study cosmic rays. 85 They study cosmic ray interactions in which the primary scattering occurs within one of the many layers of the detector. This is a big advantage, because it means the location of the vertex is known to within a few microns, the tracks of all the decay products are well measured, and therefore the pseudorapidities of the particles are known. The disadvantage, however, is that balloon borne detectors are necessarily exposed for much shorter periods of time than detectors left on mountain tops. Mountain top experiments therefore detect higher energy (rarer) cosmic rays. In fact, JACEE has seen very few events with energies in the $1000 \mathrm{TeV}$ range and no Centauros 85 However, recently 86 they have discovered events in which there are "anti-Centauro" regions in pseudorapidity-azimuth phase space containing a few tens of photons and almost no charged tracks.

Centauros are peculiar because so many charged pions are observed without any of the gammas that would indicate the decay of neutral pions. This apparent violation of isospin invariance is puzzling, unless one thinks of it in the language of a second order phase transition in which these events can be interpreted as the creation of a volume of QCD plasma in which the $\phi$ field has fluctuated throughout most of the plasma in some direction in the $\pi_{1}-\pi_{2}$ plane. This implies correlation throughout most of a volume of plasma large enough that it becomes about 100 zero temperature pions. We convinced ourselves above that this could not happen if the plasma remains close to thermal equilibrium. Hence, these Centauro events provide a tantalizing hint that it might be wise to consider the effects of going from the disordered phase above $T_{c}$ to the ordered phase rapidly without maintaining thermal equilibrium. Regardless of what the final word on Centauro cosmic ray events turns out to be, it is worth considering qualitative phenomena which can arise if the chiral transition is far from thermal equilibrium. In a heavy ion collision, even if partons reach some initial local thermal equilibrium at early times, it is far from clear that the long wavelength modes of the chiral order parameter will be in equilibrium as chiral symmetry breaking occurs.

In section 3.1, we will describe the phenomenological consequences of the existence of a large region of space in which the order parameter $\phi$ is displaced from the vacuum direction. Because of the explicit chiral symmetry breaking, $\phi$ oscillates about the $\sigma$ direction. In later sections of this chapter, we will discuss a mechanism by which these long wavelength pion oscillations could arise 1 To model the dynamics of the chiral order parameter in a far from thermal equilibrium phase transition, we will consider quenching in the $\mathrm{O}(4)$ linear sigma model. We will argue, and present numerical evidence, that in the period immediately following a quench long wavelength modes of the pion field are amplified. This could have dramatic phenomenological consequences in heavy ion collisions. Simulations of classical evolution following a quench are a long way from real heavy ion collisions. A number of authors have discussed improvements and modifications - relaxing the quench approximation; in- 
cluding effects of expansion; including quantum effects. Nevertheless, at this time a quantitative theoretical calculation is impossible. The biggest obstacle is that the appropriate initial conditions (initial meaning just before the transition occurs) on the long wavelength modes of the order parameter are not known. The physics of this chapter rests on much less solid theoretical foundations than that of the last chapter. Away from equilibrium, we cannot use renormalization group ideas or universality, and our conclusions are necessarily qualitative. If seen, long wavelength pion oscillations and the consequent large fluctuations in the neutral to charged pion ratio would be a definitive signature of an out of equilibrium chiral transition. It remains an experimental question whether or not these phenomena occur in heavy ion collisions.

\subsection{Misalignment of the Chiral Condensate}

Among the most interesting speculations regarding ultra-high energy hadronic or heavy nucleus collisions is the idea that regions in which the chiral condensate is misaligned may occur 87,88,89, 3, 90, 91 In such misaligned regions, the chiral condensate points in a different direction from that favoured in the ground state. If we parametrize the condensate using the variables of the sigma model, misaligned vacuum regions are places where the four-component field $\phi=(\sigma, \vec{\pi})$, that in the ground state takes the value $(v, 0)$, is instead partially aligned in the $\vec{\pi}$ directions. Because chiral symmetry is explicitly broken by small quark masses, the pion mass is not zero, and therefore if a misaligned vacuum region were produced, the $\phi$ field would oscillate about the $\sigma$ direction. Thus, such regions of misaligned condensate have a nonzero pion field oscillating in time. They would produce clusters of pions bunched in rapidity with highly non-Gaussian charge distributions. A misaligned vacuum region starting with the field in the $\pi_{1}-\pi_{2}$ plane would emit only charged pions (equally positive and negative, since the fields are real), while a misaligned vacuum region starting with the field pointing in the $\pi_{3}$ direction would emit only neutral pions.

More generally if we define

$$
f \equiv \frac{n_{\pi^{0}}}{n_{\pi^{0}}+n_{\pi^{+} \pi^{-}}}
$$

then each misaligned region will yield a cluster of pions with some fixed $f$. Different regions, of course, will have different values of $f$. We can calculate the probability distribution of the ratio $f$ if we assume that all initial values on the 3 -sphere are equally likely. We will make this assumption in order to get a simple analytical result

although it may not be strictly true because the quark mass term selects a preferred $\sigma$ direction even at high temperature. Where $\phi$ starts will determine in which direction it ends up oscillating about the sigma direction. We define angles on the 3 -sphere according to

$$
\left(\sigma, \pi_{3}, \pi_{1}, \pi_{2}\right)=(\cos \theta, \sin \theta \cos \varphi, \sin \theta \sin \varphi \cos \eta, \sin \theta \sin \varphi \sin \eta)
$$


Then, the ratio $f$ is given by $f=\cos ^{2} \varphi$. Under the assumption that all initial values on the 3 -sphere are equally probable, the probability distribution $\mathcal{P}(f)$ is determined by

$$
\int_{f_{1}}^{f_{2}} \mathcal{P}(f) d f=\frac{1}{\pi^{2}} \int_{0}^{2 \pi} d \eta \int_{0}^{\pi} d \theta \sin ^{2} \theta \int_{\arccos \left(\sqrt{f_{2}}\right)}^{\arccos \left(\sqrt{f_{1}}\right)} d \varphi \sin \varphi
$$

and turns out to be simply

$$
\mathcal{P}(f)=\frac{1}{2} f^{-1 / 2}
$$

Equivalently, the probability that $f<f_{1}$ is given by $\sqrt{f_{1}}$. If large regions in which $\phi$ is oscillating coherently develop in a relativistic heavy ion collision, then there should be clusters of pions with small relative momentum in which $f$ is constant, and the values $f$ takes in different such regions should be distributed according to (69).

As one application of (69), we note that the probability that the neutral pion fraction $f$ is less than .01 is 0.1 ! This is a graphic illustration of how different (69) is from what one would expect if individual pions were independently randomly distributed in isospin space. It also makes Centauro events in which less than $1 \%$ of the outgoing particles from a collision are neutral pions seem much less surprising than they first appeared. The analysis of the Centauro data is difficult for several reasons. Most important of these is the limitation imposed by small statistics. Also, if a Centauro event occurs too high above the detector, so many secondary photons will be produced that the event will not be recognized as a Centauro. Third, in a Centauro event all of the particles from the collision strike a small region of the detector and it is impossible to isolate the central rapidity region. The balloon borne JACEE detectors do not have the second and third problems, but they are only exposed during brief balloon flights and therefore have very small statistics. When relativistic heavy ion collisions in a laboratory colliding beam facility occur at high enough energies that there is a low baryon number density central rapidity region, all of the difficulties of the cosmic ray experiments will rapidly be overcome. That will be the time to look for correlated clusters of pions, and to look for a distribution like (69). In the following sections, we propose and explore a concrete mechanism by which such phenomena might arise in heavy ion collisions in which the chiral order parameter is far from thermal equilibrium. We then discuss signatures in heavy ion collision experiments.

\subsection{Emergence of Long Wavelength Pion Oscillations After a Quench}

In Chapter 2, we considered the equilibrium phase structure of QCD. We argued that QCD with two massless quark flavours probably undergoes a second-order chiral phase transition. For many purposes it is a good approximation to treat the $u$ and d quarks as approximately massless, and we expect that real QCD has a smooth but

${ }^{c}$ This result appears to have been first known in 1981,92 and has been rediscovered a number of times since then $93.87,89,89.39$ 
perhaps rapid transition as a function of decreasing temperature from small intrinsic to large spontaneous chiral symmetry breaking. At first sight it might appear that a second-order phase transition is especially favourable for the development of large regions of misaligned vacuum. Indeed the long-lived, long-wavelength critical fluctuations which provide the classic signature of a second-order transition are such regions. Unfortunately we saw that light quark masses, even though they are formally much smaller than intrinsic QCD scales, spoil this possibility. The pion masses, or more precisely the inverse correlation length in the pion channel, are almost certainly not small compared to the transition temperature. As a result the misaligned regions are modest affairs at best even near the critical temperature. They almost certainly do not contain sufficient energy to radiate large numbers of pions.

In this Chapter, we will consider an idealization that is in some ways opposite to that of thermal equilibrium, that is the occurence of a sudden quench from high to low temperatures, in which the $(\sigma, \vec{\pi})$ fields are suddenly removed from contact with a high temperature heat bath and subsequently evolve mechanically. We shall show that long wavelength fluctuations of the light fields (the pions in QCD, which would be massless if not for the quark masses) can develop following a quench from some temperature $T>T_{c}$ to $T=0$. The long wavelength modes of the pion fields are unstable and grow relative to the short wavelength modes.

In a real heavy ion collision, the phase transition must proceed by some process in between an equilibrium phase transition in which the temperature decreases arbitrarily slowly and a quench in which thermal fluctuation ceases instantaneously. Our goal is to understand the dynamics of the long wavelength modes of the chiral order parameter in a quench in the hope that the qualitative behaviour in this model is representative of processes occurring in real heavy ion collisions in which the long wavelength modes are far from equilibrium, although not quenched.

We shall use the linear sigma model of Gell-Mann and Lévyt3 to describe the low energy interactions of pions at $T=0$ after a quench:

$$
\mathcal{L}=\int d^{4} x\left\{\frac{1}{2} \partial^{i} \phi^{\alpha} \partial_{i} \phi_{\alpha}-\frac{\lambda}{4}\left(\phi^{\alpha} \phi_{\alpha}-v^{2}\right)^{2}+H \sigma\right\},
$$

where $\phi$ is a four-component vector in internal space with entries $(\sigma, \vec{\pi})$. Here, $\lambda, v$, and $H \propto m_{q}$ are to be thought of as parameters in the low energy effective theory obtained after integrating out heavy degrees of freedom. Unlike in the equilibrium case where universality allowed us to make quantitative predictions, at zero temperature we must make a non-universal choice of model Lagrangian to obtain equations of motion for the chiral order parameter. For now, we shall treat (70) as it stands as a classical field theory, since the phenomenon we are attempting to address is basically classical. We shall be dealing with energies of order $200 \mathrm{MeV}$ or less, so that neglect of heavier fields seems reasonable. Since at zero temperature the $\sigma$ is heavy - it is a feature at about $600 \mathrm{MeV}$ which is perhaps too broad to be called a resonance 
one might consider integrating it out. This would yield the nonlinear sigma model. However, we must implement initial conditions appropriate to a disordered state in which the $\vec{\pi}$ and $\sigma$ fields are equivalent up to small effects due to explicit chiral symmetry breaking. Therefore, we must keep the sigma and use (70).

To model a quench, we begin at a temperature well above $T_{c}$. The typical configurations have short correlation lengths and $\langle\phi\rangle \sim 0$. ( $\langle\phi\rangle \neq 0$ because $H \neq 0$.) One then takes the temperature instantaneously to zero. The equilibrium configuration is an ordered state with the $\phi$ field aligned in the $\sigma$ direction throughout space, but this is not the configuration in which the system finds itself. The actual, disordered configuration then evolves according to the Lorentz invariant zero temperature equations of motion obtained by varying (70). Quenching in magnet models has been much studied in condensed matter physics.94 However in that context it is usually appropriate to use diffusive equations of motion, because the magnet is always in significant contact with other light modes (e.g., phonons). For this reason the condensed matter literature we are aware of does not directly apply to our problem.

We will discuss numerical simulations of quenching to zero temperature in the linear sigma model with an explicit symmetry breaking term $H \sigma$ which makes the pions massive. Turok and Spergel 15 have considered this scenario with no explicit symmetry breaking term as a cosmological model for large scale structure formation in the early universe. They find a scaling solution in which the size of correlated domains grows without bound at the speed of light. This is easily understood qualitatively, and is peculiar to the case where $m_{\pi}=0$. The field $\phi$ is at different points on the vacuum manifold in regions of the universe which have not been in causal contact after the quench. Because the vacuum manifold is degenerate in potential energy, the system evolves to reduce gradient energy. As time continues, larger and larger regions come into causal contact, align, and the size of correlated domains grow at the speed of light. When the $O(N)$ symmetry is explicitly broken, however, we do not expect a scaling solution. The $H \sigma$ term tilts the potential, the vacuum manifold is not degenerate, and in a time of order $m_{\pi}^{-1}$ the scalar field in all regions (whether in causal contact or not) will be oscillating about the sigma direction, and the physics cannot be described in terms of differently aligned domains coming into alignment.

\subsubsection{A Numerical Simulation}

We now describe numerical simulations of quenching in the linear sigma model which were reported in Ref. [4]. We choose $\phi$ and $\dot{\phi}$ randomly independently on each site of a cubic lattice. This means that the lattice spacing $a$ represents the correlation length in the initial configuration. In the initial conditions, $\phi$ is disordered, the $O(N)$ symmetry is not spontaneously broken, and the lattice spacing $a$ represents the $\pi$ and $\sigma$ correlation lengths which are approximately degenerate. In the simulation whose results are shown in Fig. 11, we chose $\phi$ and $\dot{\phi}$ randomly from Gaussian distributions 
centred around $\phi=\dot{\phi}=0$ and with $\left\langle\phi^{2}\right\rangle^{1 / 2}=v / 2$ and $\left\langle\dot{\phi}^{2}\right\rangle^{1 / 2}=v$. The three parameters $v, H$, and $\lambda$ in (70) determine $m_{\pi}, m_{\sigma}$, and $f_{\pi}=\langle 0|\sigma| 0\rangle$ according to

$$
\begin{gathered}
\lambda\langle 0|\sigma| 0\rangle\left(\langle 0|\sigma| 0\rangle^{2}-v^{2}\right)-H=0, \\
m_{\pi}^{2}=\frac{H}{\langle 0|\sigma| 0\rangle} \quad, \quad \text { and } m_{\sigma}^{2}=3 \lambda\langle 0|\sigma| 0\rangle^{2}-\lambda v^{2} .
\end{gathered}
$$

Note that $f_{\pi}=\langle 0|\sigma| 0\rangle>v$ for $H \neq 0$. In interpreting our results we must remember that while in the code we are free to choose the energy scale by setting the lattice spacing $a=1, a$ actually represents the initial correlation length. In choosing the parameters for the simulation shown in Fig. 11, we assumed that $a=(200 \mathrm{MeV})^{-1}$ and then chose $v=87.4 \mathrm{MeV}=0.4372 a^{-1}, H=(119 \mathrm{MeV})^{3}=0.2107 a^{-3}$, and $\lambda=20.0$ so that $f_{\pi}=92.5 \mathrm{MeV}, m_{\pi}=135 \mathrm{MeV}$, and $m_{\sigma}=600 \mathrm{MeV}$.

With parameters and initial conditions chosen, we evolved the initial configuration according to the equations of motion using a standard finite difference, staggered leapfrog scheme. The simulation was performed on a $64^{3}$ lattice and the time step was $a / 10$. After every two time steps, we computed the spatial fourier transform of $\phi$. For each component of $\phi$, we computed the power

$$
P(\vec{k}, t)=\frac{1}{L^{3}}\left|\int \mathrm{d}^{3} x \exp (i \vec{k} \cdot \vec{x}) \phi(\vec{x}, t)\right|^{2}=\frac{1}{L^{3}} \int \mathrm{d}^{3} y \int \mathrm{d}^{3} x \exp (i \vec{k} \cdot \vec{x}) \phi(\vec{x}+\vec{y}, t) \phi(\vec{y}, t)
$$

and averaged over all modes with $k \equiv|\vec{k}|$ in bins of width $0.057 a^{-1} \cdot L^{3}=(64 a)^{3}$ is the lattice volume. Fig. 11 shows the power in modes of one component of the pion field with spatial wave vectors of several different magnitudes $k$ as a function of time. The curves all start at approximately the same value at $t=0$ because the initial power spectrum is white since we chose $\phi$ independently at each lattice site. For a lattice with an infinite number of sites, with the normalization used in (73) the initial value of the power spectrum is related to the mean square of the initial $\phi$ distribution by $P(\vec{k}, 0)=(1 / N)\left\langle\phi^{2}\right\rangle$, with $N=4$. This means that all the curves in Fig. 11 begin at $t=0$ at about 0.01 in lattice units. The behaviour of the low momentum pion modes is striking. While the initial power spectrum is white and, as ergodicity arguments would predict, the system at late times is approaching an equilibrium configuration in which the equipartition theorem holds, at intermediate times of order several times $m_{\pi}^{-1}$ the low momentum pion modes are oscillating with large amplitudes.

We verified that finite size effects are not important even for the longest wavelength $(k a=0.20)$ mode shown in Fig. 11 by checking that the behaviour of the $k a=0.31$ mode is the same in a $32^{3}$ and a $64^{3}$ box. The longer the wavelength of a mode, the more that mode is amplified. This holds up to the longest wavelength mode for which we can trust the results of the simulation, namely the $k a=0.20$ mode which corresponds to a wavelength $\lambda=2 \pi / k$ of about half the lattice size. 


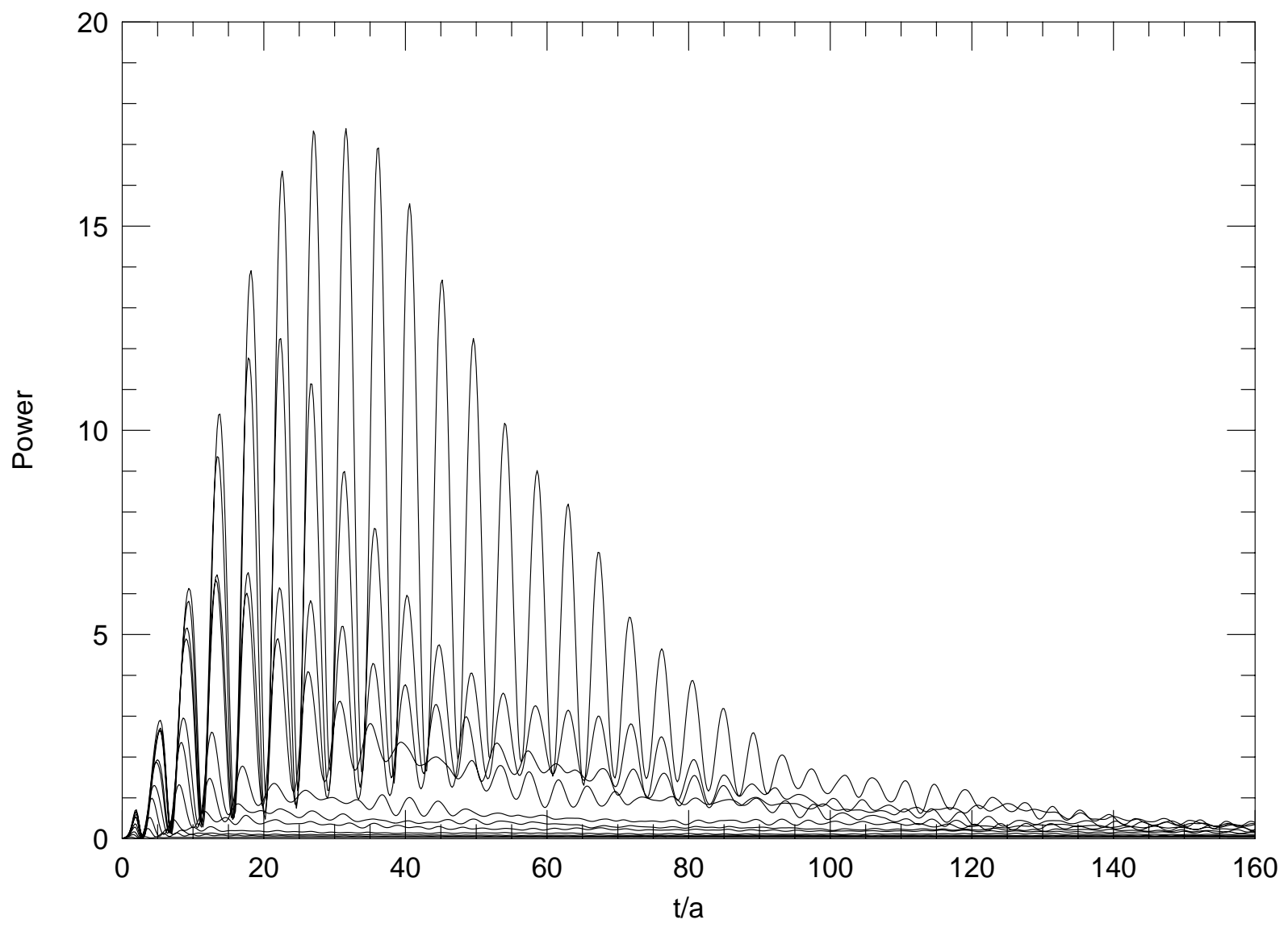

Fig. 11. The time evolution of the power spectrum of the pion field at several spatial wavelengths after a quench at $t=0$. The parameters of the potential and the initial configurations for $\phi$ and $\dot{\phi}$ are described in the text. Time is measured in lattice units, and since $a=(200 \mathrm{MeV})^{-1}$ the unit of time is approximately $1 \mathrm{fm}$. The figure shows the time evolution of the angular averaged power spectrum (defined in the text) of one of the three components of the pion field. The curves plotted are (top to bottom in the figure) the average power in the modes in the momentum bins centred at $k a=0.20,0.26,0.31,0.37,0.48,0.60,0.71,0.94,1.16,1.39$, and 1.84 . The initial power spectrum is white and all the curves start at $t=0$ at approximately 0.01 in lattice units. The power in the longest wavelength pion modes is amplified by a factor of order 1000 relative to that in the shortest wavelength modes, which is not amplified at all. From Ref. 伆; extended to later times here than in Ref. 沺. 
The behaviour of only one component of the pion field is shown in Fig. 11. The other two look qualitatively the same. It should be noted that the exact height of the peaks in the curves depend on the specific initial conditions. If the simulation is run with the same initial distributions for $\phi$ and $\dot{\phi}$ but with a different seed for the random number generator, the heights of the peaks change, and the relative sizes of the peaks in the three different pion directions change. The qualitative features of Fig. 7 - the growth of long wavelength modes of the pion field - do not depend on the specific realization of the initial conditions. Amplification occurs for a range of choices of the parameters in the potential including physically motivated values. We will discuss the effects of varying the choice of initial distributions below.

These numerical simulations have been repeated by Gavin, Gocksch and Pisarski, 96 who report that they have replicated the power spectra of Fig. 11. There has been some confusion in the literature, however. A number of authors have tried to describe the result seen in Fig. 11 in terms of a correlation length. In thermal equilibrium, this would be reasonable, as the power spectra would be completely specified by a single length - the correlation length. The essence of the phenomena of Fig. 11 is, however, that the spectra are nonthermal. They cannot be described by a single length scale, and computing a correlation length is meaningless. The classical simulation of Fig. 11 ceases to be physically relevant at late times, when instead of classical fields one has noninteracting pions. Fig. 11 has been extended formally to late times, however, to show how at late times in the classical simulation the energy is equipartitioned and thermal equilibrium is reached. Hence, comparing intermediate and late times in the figure is instructive. At intermediate times, short wavelength modes have about the same amplitude as they do at late times in thermal equilibrium, while long wavelength modes have been amplified. It is impossible to describe this behaviour in terms of a single length scale. For a visual image, think of long wavelength ocean swells with short wavelength chop in addition; perhaps it is easier just to look at Fig. 11. The essential qualitative phenomenon whose cause and consequences we now discuss is that long wavelength pion oscillations have been excited - the short wavelength modes have not been damped out. This picture is more complicated than the idealized "smooth" region of disoriented chiral condensate described in the previous section.

Although no meaningful correlation length or domain size can be defined, it is possible to describe the physics using the time dependent correlation functions

$$
C(\vec{x}, t)=\int \mathrm{d}^{3} y \phi(\vec{x}+\vec{y}, t) \phi(\vec{y})
$$

for each component of $\phi$. For each component of $\phi$, the power $P(\vec{k}, t)$ is the spatial Fourier transform of this correlation function. Since the essence of the physics we are describing is the amplification of long wavelength power, a description using the power spectrum as in Fig. 11 is the natural one, but the same information is of course encoded in the correlation function. Another reason why a description of the physics using $P(\vec{k}, t)$ is more natural than one using $C(\vec{x}, t)$ is that it is closer to what 
experimentalists measure - they measure the momenta of particles not positions in space. Long wavelength oscillations seen as amplification of $P(\vec{k}, t)$ at some small magnitude $\vec{k}$ become pions with similar momenta (in the $\hat{k}$ direction) which are close to each other in an experimentalist's detector.

\subsubsection{Understanding the Results}

The emergence of long wavelength pion oscillations in the numerical simulations is a striking qualitative phenomenon. We now discuss a simple qualitative explanation for the result. Whenever one has spontaneous breaking of a continuous global symmetry, massless Nambu-Goldstone bosons occur. The masslessness of these modes occurs because of a cancellation:

$$
m^{2}=-\mu^{2}+\lambda \phi^{2}
$$

where the second term arises from interaction with a condensate whose expectation value $\langle\phi\rangle^{2}$ satisfies $\langle\phi\rangle^{2}=\mu^{2} / \lambda \equiv v^{2}$ in the ground state. However following a quench the condensate starts with its average at 0 rather than $v$, and generally oscillates before settling to its final value. Whenever $\langle\phi\rangle^{2}<v^{2}, m^{2}$ will be negative, and sufficiently long wavelengths fluctuations in the Nambu-Goldstone boson field will grow exponentially. The same mechanism will work, though less efficiently, if one has only an approximate symmetry and approximate Nambu-Goldstone modes, or for that matter other modes whose effective $m^{2}$ is 'accidentally' pumped negative by their interactions with the condensate.

Let us compare the growth of long wavelength modes found numerically with expectations based on the mechanism just discussed. Suppose the potential $V$ in (70) were simply $V(\phi)=\left(m^{2} / 2\right) \phi^{\alpha} \phi_{\alpha}$. Then, the equations of motion would be linear, and modes with different spatial wave vector $\vec{k}$ would be uncoupled. Each curve in Fig. 11 would be a sinusoid with period $\pi / \sqrt{m^{2}+\vec{k}^{2}}$ and constant amplitude. (The power spectrum, being quadratic in the fields, oscillates with one half the period of the fields.) The period of the oscillations in Fig. 11 is indeed given by $\pi / \sqrt{m_{\pi}^{2}+\vec{k}^{2}}$, but the amplitudes are far from constant. This behaviour can be understood qualitatively by approximating $\phi^{2}$ in the nonlinear term in the equation of motion by its spatial average:

$$
\phi^{\alpha} \phi_{\alpha}(\vec{x}, t) \sim\left\langle\phi_{\alpha} \phi^{\alpha}\right\rangle(t)
$$

Note that the Hartree or mean field approximation $(76)$ is not controlled. We make it only to obtain some understanding of the qualitative behaviour of the system. Using (76) and doing the spatial fourier transform, the equation of motion for the pion field becomes

$$
\frac{d^{2}}{d t^{2}} \vec{\pi}(\vec{k}, t)=-m_{e f f}^{2}(k, t) \vec{\pi}(\vec{k}, t)
$$




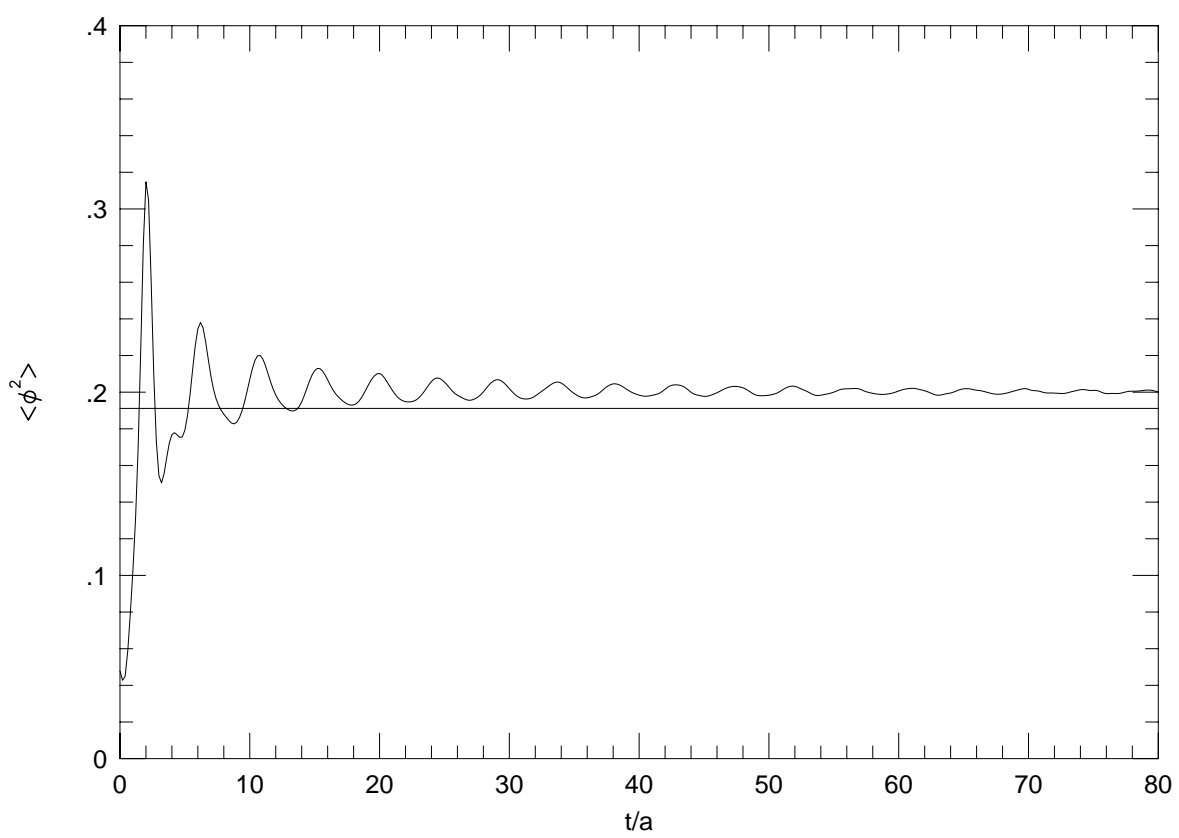

Fig. 12. Time evolution of the spatially averaged $\left\langle\phi^{2}\right\rangle$ for the same simulation whose results are shown in Figure 1. The horizontal line is at $\left\langle\phi^{2}\right\rangle=v^{2}$. From Ref. 四.

where the time dependent "mass" is given by

$$
m_{e f f}^{2}(k, t) \equiv-\lambda v^{2}+k^{2}+\lambda\left\langle\phi^{2}\right\rangle(t)
$$

and where $k=|\vec{k}|$.

Fig. 12 shows the time evolution of $\left\langle\phi^{2}\right\rangle$ in the same simulation whose results are shown in Fig. 11. In the initial conditions, $\phi$ is Gaussian distributed with $\left\langle\phi^{2}\right\rangle<v^{2}$. Therefore, for a range of wavevectors with $k$ less than some critical value, $m_{\text {eff }}^{2}<0$ and the long wavelength modes of the pion field start growing exponentially. $\left\langle\phi^{2}\right\rangle$ grows and then executes damped oscillations about its ground state value $\langle 0|\sigma| 0\rangle^{2}$. A wave vector $k$ mode of the pion field is unstable and grows exponentially whenever $\left\langle\phi^{2}\right\rangle<v^{2}-k^{2} / \lambda$. Modes with $k^{2}>\lambda v^{2}$ can never be unstable. The $k=0$ mode is unstable during the periods of time when the $\left\langle\phi^{2}\right\rangle$ curve in Fig. 12 is below $v^{2}$. Since $\left\langle\phi^{2}\right\rangle$ is oscillating about $\langle 0|\sigma| 0\rangle^{2}>v^{2}$, after some time the oscillations have damped enough that $\left\langle\phi^{2}\right\rangle$ never drops below $v^{2}$, and from that time on all modes are always stable and oscillatory. In general, longer wavelength modes are unstable for more and for longer intervals of time than shorter wavelength modes as $\left\langle\phi^{2}\right\rangle$ oscillates. Also, $m_{\text {eff }}^{2}$ is more negative and more growth occurs for modes with smaller $k$. Making the approximation (76) and thus using (77) cannot be expected to completely reproduce the effects of the nonlinear term in $(70)$ which is local in position space. Nevertheless, it predicts that the long wavelength modes of the pion field go through periods of exponential growth and predicts that the longer the wavelength the 
greater the amplification, and therefore gives us some understanding of the behaviour of these modes in Fig. 11. In an equilibrium phase transition, explicit symmetry breaking keeps the correlation length at $T_{c}$ finite and, in the case of QCD, too short to be of interest. After a quench, on the other hand, arbitrarily long wavelength modes of the pion field are amplified even though the pion mass is nonzero.

What about the sigma modes? In Ref. [四] the angular averaged power spectrum for the sigma field is plotted, as was done in Fig. 11 for one component of the pion field. For $\sigma^{2}<v^{2} / 3$, the effective mass squared for the $k=0$ mode is negative. This condition is far less likely to be satisfied than $\left\langle\phi^{2}\right\rangle<v^{2}$. Hence, while some growth of the low momentum $\sigma$ modes is possible, they do not grow nearly as much as the low momentum pion modes do.

At late times $\left\langle\phi^{2}\right\rangle$ oscillates with small enough amplitude that it never goes below $v^{2}$ and consequently no modes are ever unstable. Hence, if we make the approximation (76) we would expect that at late times each mode in Fig. 11 would continue to oscillate with approximately constant amplitude, with the longer wavelength modes maintaining the large amplitudes acquired during their exponential growth spurts. That this is not what is seen in Fig. 11 reflects the effects we neglected in making the approximation (76). Because the modes are in fact coupled and the equations of motion are actually nonlinear, ergodicity arguments suggest that eventually equipartition should apply. If the energy is equally divided among modes, then (amplitude) $)^{2}\left(m_{\pi}^{2}+k^{2}\right)$ should be approximately constant. Hence, the power in a mode at late times should be approximately constant in time and inversely proportional to $\left(m_{\pi}^{2}+k^{2}\right)$, and this is indeed the behaviour at late times in Fig. 11. It is reasonable that longer wavelength modes take longer to decrease in amplitude than shorter wavelength modes. As the system heads toward equipartition, power has to flow from low momentum modes to higher momentum modes. This plausibly happens more quickly at higher momentum because higher momentum modes are coupled to a larger number of modes, or, in the continuum, a larger volume of momentum space. Hence, we end up with the striking phenomena of Fig. 11: while the system begins and ends with small $\vec{\pi}(\vec{k}, t)$, in between the long wavelength pion modes are dramatically amplified.

The late time behaviour of the simulation in Fig. 11 is not physical. When the expansion and cooling of the plasma is taken into account, there will be a time after which the energy density is low enough that the description in terms of classical fields no longer makes sense. After this time, one has individual pions flying off towards the detector. From Fig. 11, we see that if this occurs sometime in a broad range of times of about several tens of $\mathrm{fm}$, it is plausible that at this time long wavelength modes of the pion field are greatly amplified compared to modes with higher $|k|$.

It is important to note that we have only used the Hartree approximation (76) as a qualitative guide. The emergence of long wavelength pion oscillations apparent in Fig. 11 occurs in a simulation of the strongly coupled nonlinear equations of motion. 
We have already seen that the Hartree approximation leads to incorrect conclusions about the late time behaviour in Fig. 11. In fact, the Hartree approximation can lead one astray in another way if it is taken too literally. A number of authors have noted 96 . 98 that $\left\langle\phi^{2}\right\rangle$ of Fig. 12 is less than $v^{2}$ only at early times. Indeed, in the Hartree approximation, after a time of order $m_{\sigma}^{-1} \sim 1 / 3 \mathrm{fm},\left\langle\phi^{2}\right\rangle$ is oscillating about its vacuum value. 96.98 This suggests that the growth of long wavelength modes should cease after a time of this order. However, from Fig. 11 we see that such growth occurs for much longer times - until $t \sim 30 \mathrm{fm}$. Thus, in this respect, the Hartree approximation fails to give a qualitative explanation of the phenomena observed in the full nonlinear equations. The possibility that the Hartree approximation can fail in this way was noted by Boyanovsky et al. 98 Fig. 11 is evidence that it does fail in this way.

In section 3.1, we discussed the phenomenological consequences of long wavelength oscillations of the pion field. Regions in which the oscillations are in the $\pi_{3}$ direction become clusters of neutral pions, while regions in which the oscillations are in the $\pi_{1}-\pi_{2}$ plane become clusters of charged pions. Implicit in this description was the assumption that the latter clusters include pions with both positive and negative electric charge, and are approximately neutral. We can now justify this assumption.99 While the total electric charge must be conserved, there is no conservation law forbidding the emergence of regions of net positive and negative charge. We must determine whether long wavelength oscillations of the electric charge density $\rho^{e}$ occur. The electromagnetic current due to the charged pions is given by

$$
j_{\mu}^{e}=\pi_{1} \partial_{\mu} \pi_{2}-\pi_{2} \partial_{\mu} \pi_{1}
$$

so the charge density is

$$
\rho^{e}=\pi_{1} \dot{\pi}_{2}-\pi_{2} \dot{\pi}_{1}
$$

It is easy to verify that the equations of motion obtained by varying $(70)$ do indeed conserve $j_{\mu}^{e}$. Because $j_{\mu}^{e}$ is conserved, we expect it to be more difficult to excite long wavelength charge oscillations than to excite long wavelength oscillations of $\pi_{1}, \pi_{2}$, or $\pi_{3}$. If we write $\left(\pi_{1}, \pi_{2}\right)$ as a complex field $r \exp (i \theta)$ then $\rho^{e}=r^{2} \dot{\theta}$. We have seen that there are periods of time when the pion field is exponentially unstable. This instability which manifests itself dramatically in Fig. 11 results in growth of the magnitude of $\vec{\pi}$. Thus, it leads to growth of $r$ but does not tend to change $\theta$. Equivalently, the instability tends to kick $\vec{\pi}$ radially away from $\vec{\pi}=\overrightarrow{0}$, and results in oscillations with $\vec{\pi}$ oscillating approximately through the origin with $\dot{\theta} \sim 0$ rather than with $\vec{\pi}$ "orbiting" the origin with $\dot{\theta} \neq 0$. We have verified that the dramatic phenomena of Fig. 11 are not manifest in the electric charge density $\rho_{e}$ by computing the spatial fourier transform of $\rho^{e}$ in the simulation of Fig. 11. First, we have verified that the total charge in the $64^{3}$ box is indeed conserved. Second, we found that the low $k$ modes of $\rho^{e}$ do not grow dramatically. Long wavelength oscillations of $\pi_{1}, \pi_{2}$, 
and $\pi_{3}$ are dramatically amplified after a quench, while long wavelength oscillations of the conserved electric charge density $\rho_{e}$ are relatively unaffected.

\subsubsection{Other Scenarios}

Bjorken, Kowalski, and Taylor have proposed that regions of disoriented chiral condensate may arise in proton-proton collisions. 99. 90.:91 They suggest that after a collision, a "cold" region throughout which the condensate is misaligned in a similar direction in isospin space is surrounded by a "hot" region of outgoing particles hence the name Baked Alaska for the scenario. The MiniMax experiment 100 at Fermilab (J. D. Bjorken and C. C. Taylor, co-spokespersons) is currently taking data to search for such phenomena. The scenario we have described is somewhat different, in that while long wavelength oscillations of the chiral condensate arise, we do not envision "smooth" regions of disoriented condensate. The long wavelength sloshing, although dramatic because it is completely different than what is obtained in thermal equilibrium, nevertheless has short wavelength oscillations superimposed on top of it. The emergence of long wavelength pion oscillations as described in this article requires that a large volume of space (certainly several fm across) is first in a high temperature phase in which the condensate is disordered ( $n . b$ disordered not disoriented). The volume has to be large enough that it makes sense to discuss long wavelength modes of the chiral order parameter. In this volume, the plasma must then experience a transition to the low temperature phase which occurs in such a fashion that the long wavelength modes of the order parameter do not stay in thermal equilibrium. We suggest that this physics may be relevant in the central rapidity region of a sufficiently energetic heavy ion collision, but it is not clear that it is possible to use this sort of language to describe a proton-proton collision. Although the Baked Alaska scenario is somewhat different than the one we are discussing, it has similarities. It is certainly far from thermal equilibrium. Furthermore, Bjorken 101 has suggested that the processes at the boundary between the "hot" and "cold" regions of the Baked Alaska can be described as a quench. Finally, and most important, although the settings are different - hadron-hadron collisions vs. heavy ion collisions - at the end of the day long wavelength pion oscillations lead to large fluctuations in the neutral to charged pion ratio in either case.

The idea that coherent pion emission from a classical pion field occurs in collision experiments has a long history, 102, $97,103,92.93$ and solutions to the classical equations of motion have been studied in other contexts also.104 In addition to the ideas we have described to this point, another possibility which has been discussed in the literature is that when there are a lot of pions in a small volume the fact that they are bosons can play a role — bose condensation can occur, 105 and the wave function must be symmetrized 100 This physics can have the effect of broadening the probability distribution for $f$ from a narrow Gaussian, although it does not lead to a $1 / \sqrt{f}$ 
distribution. Pehaps this could be associated with Centauro events. Greiner, Gong and Müller 107 have argued that for these effects to be significant, pion densities larger than those likely to occur at RHIC are necessary, and that in addition if such large densities were to occur, a description in terms of a bose gas of pions would not be appropriate. There have also been recent proposals that classical pion fields could arise in hadron-hadron collisions 108,109 and in the leading (high-rapidity) regions of heavy ion collisions 109

It may turn out that the amplification of long wavelength modes described in this article may not be the appropriate explanation for the Centauro events, which may turn out to be hadron-hadron or hadron-nucleus collisions in which a large volume in which the chiral order parameter in its high temperature phase is never formed. Related ideas like Bjorken, Kowalski, and Taylor's Baked Alaska scenario for the formation of a region of disoriented chiral condensate, or some of the other ideas just mentioned may turn out to be more appropriate descriptions of Centauro events, and the interesting phenomena which we hope Bjorken, Taylor, et al. discover experimentally 100 in proton-proton collisions.

In studying the chiral phase transition, perhaps the role of the Centauro events will prove to be merely inspirational, and not of direct relevance. Whatever the inspiration, it is important to consider the qualitative phenomena which are possible when the plasma in the central rapidity region of a heavy ion collision goes from the high temperature phase in which the chiral order parameter is disordered to the low temperature phase in such a way that long wavelength modes of the order parameter do not stay in equilibrium. We have used the quench as an idealization which may capture the relevant non-equilibrium physics. We have seen numerical evidence (supported by qualitative understanding) that long wavelength oscillations of the order parameter in the pion directions may be greatly amplified, whereas this does not happen if thermal equilibrium is maintained. In the next sections, we describe various improvements to the treatment, and discuss experimental consequences.

\subsection{Initial Conditions, Expansion, and Quantum Effects}

The results shown in Fig. 11 do depend on the initial distributions for $\phi$ and $\dot{\phi}$. If the initial conditions are such that the energy per unit volume in the simulation is much more than $\frac{\lambda}{4} v^{4}$, no striking growth of long wavelength modes occurs. The system quickly achieves a configuration in which the energy is equipartitioned among modes corresponding to thermal equilibrium at a temperature above $T_{c}$. Thus, in order for long wavelength modes to grow as in Fig. 11, the energy of the fluctuations in $\phi$ in the initial conditions must be less than it would be in thermal equilibrium. Another way of saying this is that whereas in the initial condition for Fig. $11\langle\phi\rangle$ is in a configuration

appropriate to a high temperature, $\left\langle\phi^{2}\right\rangle$ and $\left\langle\dot{\phi}^{2}\right\rangle$ have magnitudes characteristic of a lower temperature. These admittedly ad hoc quench initial conditions were chosen in 
the hope of capturing qualitative behaviour typical of more general nonequilibrium physics. We now discuss efforts to improve upon this treatment.

Gavin and Müller 110 note that if upon starting with quench initial conditions, the condensate were to evolve from $\langle\phi\rangle \sim 0$ to $\langle\phi\rangle \sim v$ much more slowly than it does in the classical linear sigma model considered above, then the amplification of long wavelength pion oscillations would be much greater than in Fig. 11. They call this scenario annealing, although this is a somewhat different usage than in condensed matter physics. Annealing usually means slow cooling in which both condensate and fluctuations stay close to their thermal equilibrium configurations as the temperature is slowly reduced. In this standard annealing, since approximate thermal equilibrium is maintained, no growth of long wavelength modes would occur. Gavin and Müller's scenario, in which the condensate evolves slowly (as in standard annealing) but in which nonequilibrium conditions which allow amplification of long wavelength modes are maintained nevertheless, is in a sense more out of equilibrium than a quench. It would be marvelous if this turned out to be the appropriate idealization for treating heavy ion collisions, but that may be too much to hope for.

A much more ambitious project than the classical simulations described so far is to include the effects of quantum fluctuations (in addition to classical thermal fluctuations) on the dynamics of the order parameter. A number of groups have made progress in this direction.111, 80.112 In order to treat the quantum fluctuations, however, all groups have had to make linearizing approximations either by working in the Nambu-Jona-Lasinio model, 111 or by using the Hartree approximation 98 or the large-N approximation. 12 Such approximations cannot be avoided at present, but we have already seen that they are fraught with risk. Using the Hartree approximation to study the classical simulations of Fig. 11 leads to the prediction that growth of long wavelength modes occurs for a much shorter time than is in fact the case. Thus, it is perhaps not surprising that in treatments of a quench in which quantum effects have been included, short correlation lengths have been found.11.98 Also, as we have noted before, the essence of the phenomenon discovered in the classical simulations cannot be described simply as the development of a long correlation length. Long wavelength modes are amplified, while short wavelength oscillations remain. Further work is needed in order to understand quantum effects on the dynamics.

A less ambitious improvement on the simulations of the previous section is to include the effects of expansion. Following Bjorken, 31 we wish to implement the boost invariant longitudinal expansion characteristic of the central rapidity region of a sufficiently energetic heavy ion collision. The natural coordinates to use are the rapidity $y$ of equation (21) and the proper time $\tau$ of equation (22), instead of the time $t$ and the longitudinal position $z$. This can be implemented by taking the linear sigma model equations of motion and performing the replacement

$$
\left[\frac{\partial^{2}}{\partial t^{2}}-\frac{\partial^{2}}{\partial z^{2}}\right] \rightarrow\left[\frac{1}{\tau} \frac{\partial}{\partial \tau}\left(\tau \frac{\partial}{\partial \tau}\right)-\frac{1}{\tau^{2}} \frac{\partial^{2}}{\partial y^{2}}\right]
$$


The first treatments to include expansion were $1+1$ dimensional - they assumed the configuration was uniform in the transverse directions. 88, 113, 114, 115, 116, 117, 118 By making the further simplifying assumption that $\phi$ is independent of rapidity, Blaizot and Krzywickis 114 reduce the problem to a $0+1$ dimensional nonlinear dynamical system. Following the evolution of these equations is instructive for analyzing the physics of a large smooth disoriented chiral condensate as envisioned in the Baked Alaska scenario of Bjorken et al., and is a good toy model in which calculations can be done without resorting to numerical work. ${ }^{d}$ Krzywicki13 has considered how the dynamics of this simple system can be affected by coupling to a bath representing all the other degrees of freedom in the system. Huang and Wang 117 have done a $1+1$ dimensional simulation in which $\phi$ and $\dot{\phi}$ are random functions of rapidity with the same quench initial distributions that were used in the simulation of Fig. 11. They find that oscillations in the $\pi$ fields as a function of rapidity develop over distances in rapidity of up to 2 or 3 .

One reason why it is important to include expansion is that one can then ask whether it is possible to begin with initial conditions in thermal equilibrium at a high temperature and have something analogous to a quench occur as the system cools by expanding. This question has been addressed in numerical simulations by Asakawa, Huang, and Wang 119 and by Cooper et al.112 We will describe the work of both groups below, but let us first consider the qualitative answer to the question just posed. Both groups find that starting with thermal equilibrium, one stays close enough to thermal equilibrium that no significant growth of long wavelength modes occurs. Both groups also find, however, that if the long wavelength modes of the condensate are not in thermal equilibrium at the start of their simulations, then long wavelength pion oscillations can, in fact, be amplified. It would have been wonderful if simply adding expansion to the linear sigma model had been enough to turn thermal equilibrium into a quench, but perhaps this was too much to ask. Real heavy ion collisions are much more complicated than the linear sigma model, however. Indeed, using the sigma model to describe the dynamics cannot be valid for $T \gtrsim T_{c}$. The unanswered question can be phrased as follows: In a heavy ion collision, at the time when it becomes appropriate to use the linear sigma model to describe the dynamics, are the long wavelength modes of the order parameter in thermal equilibrium with the short wavelength modes and the other degrees of freedom, or are they out of equilibrium? Nobody knows the answer. If they are out of equilibrium, then perhaps the quench discussed above or the quench with expansion to be discussed below yield

\footnotetext{
${ }^{d}$ Some authors 8780101 have used the further simplification gained by working in the nonlinear sigma model in which there is no $\sigma$ degree of freedom. In this article, we are interested in the amplification of long wavelength pion oscillations from an initial state in which $\phi$ is disordered and the $\vec{\pi}$ and $\sigma$ fields are equivalent up to small effects due to explicit chiral symmetry breaking. We have therefore had to use the linear sigma model. If, however, one is interested in studying the evolution of long wavelength pion oscillations at later times without regard to how they may have arisen at earlier times, then the nonlinear sigma model suffices.
} 
a qualitative description of the physics. If that is the case, then long wavelength pion oscillations arise.

Asakawa, Huang, and Wang 119 have simulated the classical equations of motion of the linear sigma model in $2+1$ dimensions, where the two spatial dimensions are the transverse ones. They include the effect of longitudinal expansion by using $(1 / \tau) \partial / \partial \tau(\tau \partial / \partial \tau)$ instead of $\partial^{2} / \partial t^{2}$ in the equations of motion, but they do not do a $3+1$ dimensional simulation. They are therefore able to use a large 2-dimensional lattice, and can implement transverse expansion. They do so by choosing initial conditions which are vacuum outside a cylindrical region of transverse radius $5 \mathrm{fm}$, and with $\phi$ disordered with a correlation length of $0.5 \mathrm{fm}$ and with $\langle\phi\rangle=0$ inside the cylindrical region. They do two simulations. The first has $\left\langle\phi^{2}\right\rangle$ large enough to approximate thermal initial conditions, and not much happens in this simulation. The second simulation, shown in Fig. 13, has $\left\langle\phi^{2}\right\rangle$ smaller, as in a quench. The transverse expansion is apparent in the figure, as is the growth of long wavelength pion oscillations. Just as in Fig. 11, one obtains a configuration with prominent long wavelength oscillations (about $4 \mathrm{fm}$ from peak to trough; hence about $8 \mathrm{fm}$ in wavelength) superimposed with short wavelength oscillations which have not been damped out. As in the simulations of Ref. [1], the longest wavelengths which are amplified are comparable to the system size.

The linear sigma model simulations of Cooper et al. .12 are $3+1$ dimensional, include the effects of quantum fluctuations, include the time dependent renormalization of the couplings in the potential due to the changing quantum and thermal fluctuations, include boost invariant longitudinal expansion, and do not include transverse expansion. In order to include quantum effects, they work in a mean field approximation, and so risk missing some of the physics as we discussed in section 3.2.2. Because longitudinal expansion is included, the amplitudes of all the fields becomes small at very late times, the equations of motion linearize, and it becomes possible to define and measure particle numbers. Results from their simulations are shown in Fig. 14. They plot the mean number density $n(p)$ and its fluctuations as a function of transverse momentum $p$ at several proper times. (See Ref. 112 for definitions, but growth of $n(p)$ for low $p$ corresponds to growth of long wavelength oscillations.) The left column is for a simulation with thermal initial conditions with an initial temperature of $T=200 \mathrm{MeV}>T_{c}$. Because of the longitudinal expansion, the system cools through $T_{c}$, but long wavelength modes are not amplified. The right column is for a simulation with nonthermal initial conditions in which $\langle\sigma\rangle$ has been given an initial nonzero time derivative. Cooper et al. note that using quench-like initial conditions (long wavelength modes in a thermal distribution at one temperature with short wavelength modes in a thermal distribution at a lower temperature) produces similar results to those shown in the right column. Long wavelength oscillations are dramatically amplified. It is impossible to define a domain size at late times in the right column, because multiple length scales have emerged. For nonthermal initial 


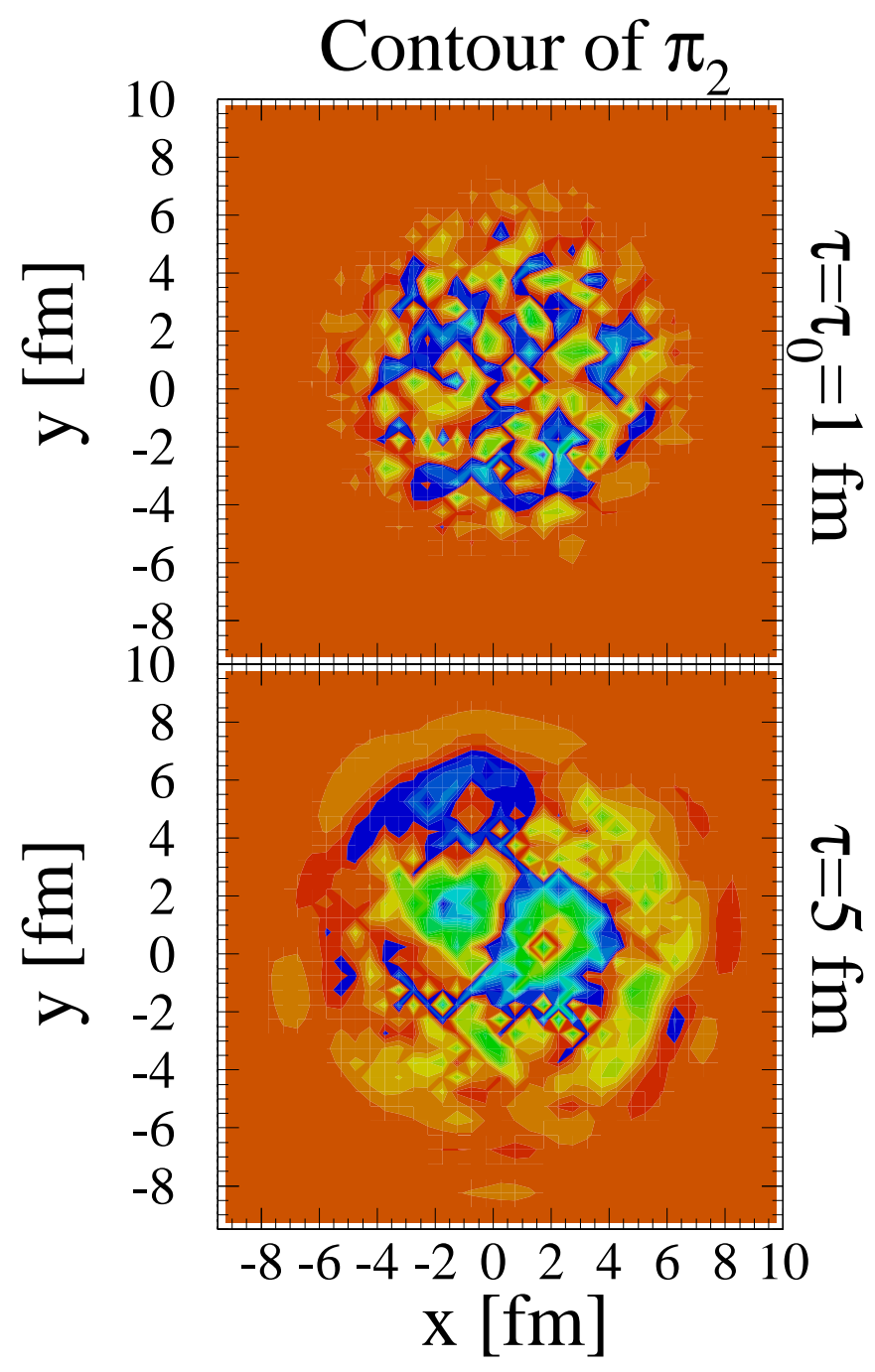

Fig. 13. From Ref. [119]. Contour plot of a component of the $\pi$ field $\left(\pi_{2}\right)$ at the initial proper time $\tau=1 \mathrm{fm}$ and later at $\tau=5 \mathrm{fm}$ in a $2+1$ dimensional simulation of the linear sigma model including expansion with quench initial conditions done by Asakawa et al. The uniform grey around the outside of both figures represents $\pi_{2}=0$. In the lower panel, there are prominent features at longer wavelengths than in the initial conditions shown in the upper panel. For example, the structure centred at about $(2,0)$ is a region of positive $\pi_{2}$ peaking at about $85 \mathrm{MeV}$, and the structure centred at about $(-1.5,1.5)$ is a region of negative $\pi_{2}$ with a minimum at about $\pi_{2}=-65 \mathrm{MeV}$. These structures are not smooth, however. (The grey-scale in this black and white representation of the figure does not make all this apparent - the original in Ref. [119] is in colour, and the authors also have a three dimensional colour version which is the clearest way of presenting the results, but which is not clear in black and white.) Thus, prominent long wavelength oscillations with wavelengths of about $8 \mathrm{fm}$ have been excited. Because of the cylindrical boundary conditions used to implement the transverse expansion, these results cannot simply be Fourier transformed, as was done in producing Fig. 11. However, the phenomena are clearly analogous. Long wavelength modes have been markedly amplified, but they cannot be described as smooth domains as they have short wavelength oscillations superimposed on them. 

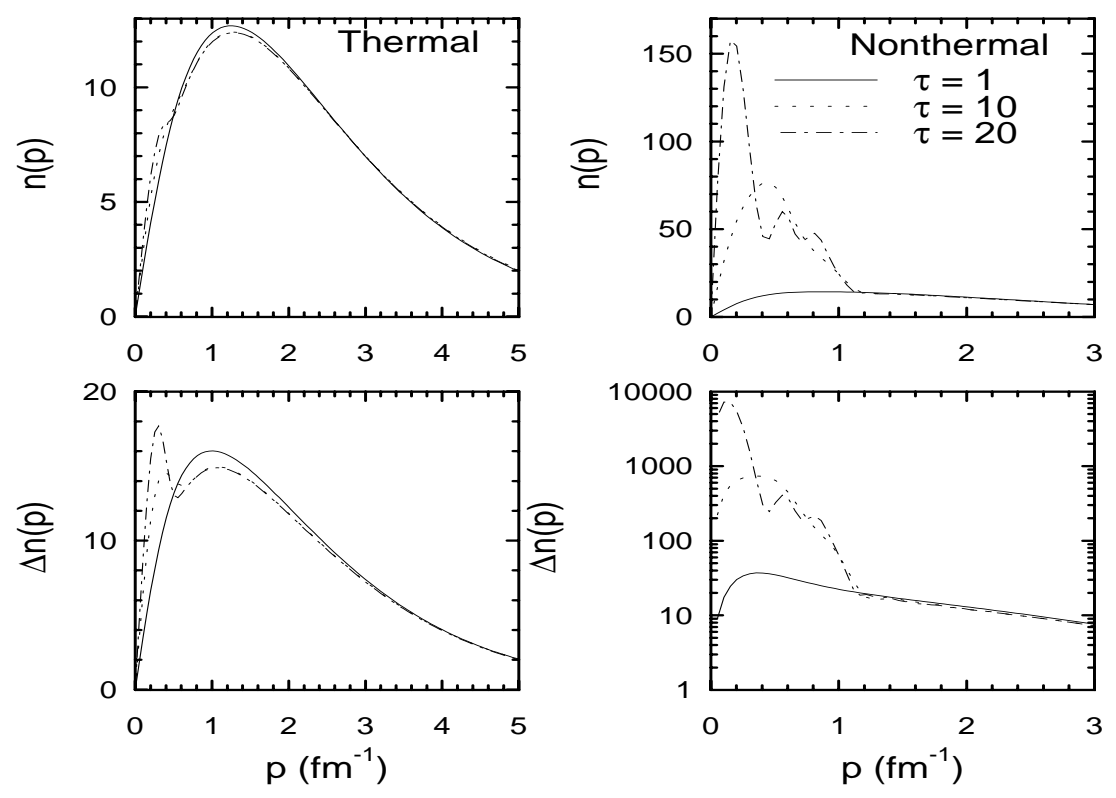

Fig. 14. From the final paper of Ref. [112]. Results from simulations by Cooper et al. of the linear sigma model in a mean field approximation including quantum and thermal fluctuations and boost invariant longitudinal expansion. For nonthermal initial conditions, long wavelength oscillations are amplified, while this does not occur for thermal initial conditions. See text for further discussion.

conditions, amplification of long wavelength modes is manifest in the particle number distribution, while for thermal initial conditions, this does not happen. This result is in qualitative agreement with that of Asakawa et al. 119 and confirms the observation first made in Ref. [4]: if the long wavelength modes of the order parameter are not in thermal equilibrium as the chiral transition occurs, there is a simple mechanism which can lead to dramatic amplification of long wavelength pion oscillations.

To this point, the only quantum effects we have discussed are the quantum corrections to the dynamics. There are several other inherently quantum mechanical questions, however. First, once a long wavelength oscillation has been created, at late times as the classical treatment is becoming inappropriate one might worry that there may be enough quantum scattering of pions $\left(e . g \pi^{0} \pi^{0} \rightarrow \pi^{+} \pi^{-}\right)$to wash out the effect of interest before the pions are far enough apart on their way to the detector that they no longer interact. This question is being studied by Huang and Suzuki, 120 and their preliminary result is that such effects are small because pions, as Goldstone bosons, have only derivative couplings and so have small scattering rates at low momentum.

Another very interesting question which has received much attention is: To what quantum state does a classical configuration in which the chiral condensate is misaligned and oscillating correspond? In other words, once the oscillating classical field becomes noninteracting quantum mechanical pions, what is the quantum state of those pions? Following Horn and Silver, 97 a number of authors3. 
ered the unique quantum state with $N$ pions with zero total isospin. Computing the distribution of the number ratio $f$ of neutral pions obtained in this state does indeed yield the $(1 / 2) f^{-1 / 2}$ distribution. Greiner, Gong, and Müller note that this state also has unusual correlations. Defining

$$
\mathcal{C}_{\alpha \beta} \equiv \frac{\left\langle n_{\pi^{\alpha}} n_{\pi^{\beta}}\right\rangle}{\left\langle n_{\pi^{\alpha}}\right\rangle\left\langle n_{\pi^{\beta}}\right\rangle}-1
$$

where $\alpha$ and $\beta$ can be $0,+$, or - , they find that for states with many pions, $C_{00}=$ $4 / 5, C_{0+}=C_{0-}=-2 / 5$ and $C_{++}=C_{--}=C_{+-}=1 / 5$. Note that in ordinary intensity interference experiments for bosons coherent sources give $C=0,121$ and $C \neq 0$ is only possible for identical bosons. Here, in contrast, neutral and charged pions are each positively correlated, and anti-correlated with the other. It seems likely that these charge dependent correlations will be qualitatively similar for other quantum states which may correspond to classical regions in which the condensate is misaligned. While it is reasonable to consider states with small isospin, there is no reason to require $I \equiv 0$. It is therefore reassuring that Cohen et al. 122 have shown that the $(1 / 2) f^{-1 / 2}$ distribution is significantly affected only when $I$ becomes larger than about $30 \%$ of the number of pions. Once one allows consideration of quantum states which do not have a definite particle number, there are many possibilities. Amado and Kogan 123 have enumerated several, including the isospin projected coherent

states of Botke, Scalapino, and Sugar 103 and squeezed states. 125 Other authors 98.112 have noted that mixed states described by density matrices are a possibility. The argument for the relevance of squeezed states 123 is an interesting one. In the Hartree approximation, each mode of the pion field satisfies the equations of motion (77) for a harmonic oscillator with a time dependent mass. Replacing the classical modes by quantum harmonic oscillators, one obtains parametric quantum oscillators, and this is just the setting in which squeezed states naturally arise 125 The argument does rely on using the Hartree approximation, which we already know to be problematic, and so it is not conclusive. The question of what quantum state corresponds to a region in which long wavelength amplifications of a classical field remains open.

\subsection{Signatures and Experiments}

The definitive signature of long wavelength pion oscillations is large fluctuations in $f$, the neutral pion fraction, and we discuss this below. However, a number of other signatures have also been proposed. Huang, Suzuki, and Wang 126, 127 have noted that in a region in which the chiral condensate is misaligned, the isovector part of the electromagnetic current is no longer dominated by the $\rho^{0}$ meson as in the normal vacuum - it couples both to $\rho$ mesons and to their chiral partners the $a_{1}$ mesons. Huang et al. consider a large smooth region of disoriented vacuum, and average over all possible directions of disorientation, and find that the $\rho$ peak at $770 \mathrm{MeV}$ in the dilepton spectrum will be reduced by a factor of 2 and a broad bump in the dilepton 
spectrum will emerge at the $a_{1}$ energy $(1260 \mathrm{MeV})$. The $\omega$ meson, on the other hand, is a chiral singlet and so will not be affected. They therefore propose that seeing a reduction in the $\rho$ peak in the dilepton spectrum while the $\omega$ peak remains unchanged would be a signal for the formation of disoriented chiral condensate. It is not clear, unfortunately, that this signal would be prominent in the more realistic situation in which there are long wavelength oscillations of the condensate, but not large smooth regions.

A direct signal of the amplification of long wavelength pion oscillations would be simply to look for an enhancement of the number of pions produced at low momentum, without regard to their charge.228.227 It is not clear that such a signal would be unambiguous in and of itself. However, if this signal were detected in coincidence with the detection of large fluctuations in $f$, then it might provide a estimate of the wavelengths of importance - excess production of pions below some transverse momentum $p_{T}$ would show that modes with wavelengths longer than $1 / p_{T}$ had been amplified.

It is also worth looking for the correlations $\mathcal{C}_{\alpha \beta}$ defined in (82). In particular 107 the unusual charge dependence of the correlations (neutral and charged pions correlated with themselves, and anti-correlated with the other) may be detectable. Gavin 128 has also considered distinguishing the effect of regions of disoriented chiral condensate on the correlation between like sign charged pions from that of standard interference effects, but concludes that this will be difficult.

We now turn to the definitive signature of long wavelength oscillations of the chiral order parameter. If a particular $\vec{k}$ mode ends up oscillating in the $\pi_{0}$ direction, then it will become neutral pions moving in the direction of $\vec{k}$. Similarly, oscillations in the $\pi_{1}-\pi_{2}$ plane will become charged pions. Thus, in some regions of the detector, $f$ would be large, and in others it would be small. If this were the only physics occurring, $f$ would be distributed like $(1 / 2) f^{-1 / 2}$ as we have discussed, but of course there will be a large background due to all the other pions in the event. (These correspond very roughly to the short wavelength oscillations which, as we have seen, remain even as long wavelength oscillations are amplified. This correspondence is only rough, however, because at short wavelengths the linear sigma model cannot capture all of the physics.)

What is needed is a detector which covers as much of $4 \pi$ solid angle as possible, which counts both charged pions and photons from the decay of neutral pions, and which is segmented in pseudorapidity $\eta$ and azimuth $\varphi$ into fine enough segments that the number of photons and charged pions in each segment can be accurately measured. (Ideally, a theorist's detector would be segmented enough that the typical segment had either zero or one photon or charged pion in it.) One would use this detector to look at the central rapidity region of the collision, where almost all the charged particles are pions and where almost all the photons come from neutral pion decay, so that particle identification is not crucial. It would be very helpful to have enough 
information about the transverse momenta $p_{T}$ so that high momentum charged pions and photons can be cut from the data. (In looking for a signal, one of the things to play with would be where to put the $p_{T}$ cut, but somewhere around $200 \mathrm{MeV}$ would seem reasonable.) The data, then, would consist of an event by event catalogue of the positions in $(\eta, \varphi)$ of the photons and charged pions. There are many conceivable ways of analyzing the data to look for large fluctuations in $f$. Several are described below.

Let us first make a very crude attempt to estimate the number of pions that might arise from the excitation of a long wavelength pion oscillation. Inspired by Fig. 13, consider a wave with wavelength $\lambda \sim 8 \mathrm{fm}$ and with $k=2 \pi / \lambda \sim 150 \mathrm{MeV}$. The energy density of such an oscillation with amplitude $A$ is

$$
\varepsilon \sim\left(m_{\pi}^{2}+k^{2}\right) A^{2} \sim 25\left(\frac{A}{f_{\pi}}\right)^{2} \mathrm{MeV} / \mathrm{fm}^{3} .
$$

The number of pions, then, is

$$
N \sim \frac{V \varepsilon}{\sqrt{m_{\pi}^{2}+k^{2}}} \sim 125\left(\frac{V}{(8 \mathrm{fm})^{3}}\right)\left(\frac{A}{f_{\pi}}\right)^{2}
$$

where $V$ is the volume of space in which this mode is oscillating with this amplitude. The uncertainties in this estimate are huge. There is no information in the simulations of Fig. 13 about the longitudinal extent of the region which is oscillating. However, if it extends in rapidity from -1 to 1 , then at a time $t=5 \mathrm{fm}$ this corresponds to an extent in $z$ of $8 \mathrm{fm}$. So, in (84) we have taken $V \sim(8 \mathrm{fm}) \lambda^{2}$. Note, however, that there is no reason why $V$ cannot be larger than $\lambda^{3}-V$ is the volume over which the mode is excited, and is not in principle related to $\lambda$. We do not really know what amplitudes can arise - $A \sim 75 \mathrm{MeV}$ in Fig. 13, and in general it seems reasonable that $A \sim f_{\pi}$ is possible. Gavin has also tried to make an estimate of the number of pions from an event like that in Fig. 13,128 he comes up with 30-300 pions, which seems reasonable. Following Horn and Silver, 97 Gavin 128 notes that the momentum distribution of pions emitted from a smooth region of disoriented condensate of radius $R$ goes like $\exp \left(-p^{2} R^{2}\right)$. Taking $R=\lambda / 2$, this suggests typical transverse momenta of about $50 \mathrm{MeV}$.

A few words of warning about the estimates just given seem in order. A reader at this point may be crying out that we should do a better job of analyzing the simulations done by various authors, and do better simulations, to make the estimates of the typical transverse momenta and pion multiplicity more accurate. This would be misleading. The theoretical foundations of the work described in this chapter are very speculative. Using the linear sigma model to describe the dynamics seems reasonable for the long wavelength modes, but cannot be justified in a controlled way. Either neglecting quantum effects or including them in a Hartree approximation risks 
missing some of the physics. Most important of all, we do not know what initial conditions to impose on the long wavelength modes of the chiral order parameter at the time when partonic language ceases to be appropriate and a description of the dynamics in terms of the linear sigma model becomes appropriate. Thus, estimates of the kind given above are necessarily very crude. Indeed, the best advice for an experimentalist is to ignore all attempts at quantitative estimates, and just try to look at what is out there. Furthermore, although there are fewer complications at RHIC than at current lower energy experiments, given the lack of theoretical certainty it would be crazy not to look for fluctuations in $f$ in current experiments at CERN and at the AGS. This is being or will soon be done, 129 but no results have yet been reported.

Let us now consider how future data might be analyzed. The first thing to do will be simply to plot the data on a lego plot (i.e. in the $(\eta, \varphi)$ plane) using one colour for charged particles and another for photons, and then simply look by eye to see what is there. One of the consequences of the amplification of long wavelength pion oscillations is that rare dramatic events in which regions of the lego plot are almost all neutral or almost all charged will be much less rare than in thermal equilibrium. As Krzywickit1 has emphasized, even if such dramatic events remain rare, (i.e. less rare than in equilibrium but rare nevertheless) they are worth waiting for and looking for.

Another simple way of analyzing the data will be to count all the charged particles and all the photons in each event, compute $f \sim \frac{n_{\gamma} / 2}{n_{\text {charged }}+n_{\gamma} / 2}$ for each event, and plot a histogram of the $f$ 's for different events. 130 As an example, the WA98 experiment 131 at CERN detects both photons and charged particles in a window of rapidity of width 1.1 near central rapidity, over all $2 \pi$ of azimuth. In a typical event, of order $600-800$ charged particles are accepted. The task will be to compare the $f$ histogram from the data with the $f$ histogram for "background" events - presumably Monte Carlo generated - which do not contain any unusual fluctuations in $f$ and so have a Gaussian $f$ distribution with width proportional to the mean value of $\left(n_{\gamma} / 2+n_{\text {charged }}\right)^{-1 / 2}$. Long wavelength oscillations of the chiral order parameter would lead to a broadening of this histogram. Clearly, understanding the $f$ distribution of the background events is crucial. There is reason for optimism, however. It seems likely that if a Monte Carlo event generator predicts, say, more charged pions than actually occur, then it will also predict more neutral pions than actually occur, while the $f$ distribution will be unaffected. This suggests that $f$ distributions for background events obtained from event generators will be much more robust and accurate than, say, distributions for the total multiplicity. This must of course be studied and tested further. Preliminary work suggests that a population of events given by taking Monte Carlo generated background events, superimposing 150 pions with momenta distributed like $\exp \left(-(p / 50 \mathrm{MeV})^{2}\right)$ in the centre of mass frame, uniformly distributed in azimuth, and with $f$ distributed like $(1 / 2) f^{-1 / 2}$, and then imposing WA98 acceptances, would 
yield an $f$ histogram which is clearly detectably broader than that of a population of background events. It might also be helpful to look for broadening of the $f$ histogram as a control parameter is varied which changes the energy of the collision. The beam energy is the obvious choice, but if that is not possible, looking at events with varying impact parameter may prove useful. At RHIC, the analysis analogous to the one sketched above would be to average over all charged particles and photons in a rapidity bin of some width, say 1, rather than to average over all particles in an entire event.

It is important to realize, however, that averaging over azimuth is pessimistic. It is entirely possible that in a given event (or in a given region of rapidity in an event) long wavelength modes corresponding to one direction in azimuth will end up oscillating in one direction in $\vec{\pi}$ space, while modes corresponding to a different direction in azimuth oscillate in a different direction in $\vec{\pi}$ space. Stated in another way, it is clear that the pions from the long wavelength $\pi_{2}$ oscillation in Fig. 13 will not be uniformly distributed in azimuth. Thus, the generic signature of long wavelength pion oscillations is not large fluctuations of $f$ which are uniform in $\varphi$, but rather large fluctuations in $f$ which depend both on $\varphi$ and $\eta$. Thus, the data should be binned in bins of varying extents $\Delta \eta$ and $\Delta \varphi$, and $f$ histograms should be done for varying bin sizes. Again, for each bin size, the $f$ histogram from the data must be compared with that from background events.

Another way of organizing the data analysis goes as follows. 132 Bin the data in such small bins that there are, say, about 10 particles per bin. Then for a bin centred at $(\eta, \varphi)$ define $\tilde{f}(\eta, \varphi)=f-\langle f\rangle$. (In a real experiment, once experimental acceptances are taken into account, $\langle f\rangle$ will not be precisely $1 / 3$.) Because $\tilde{f}$ is defined using very small bins, a histogram of $\tilde{f}$ would be broad due to fluctuations even in the absence of interesting effects, and so would not be useful. However, compute the correlation function

$$
C(\Delta \eta, \Delta \varphi)=\left\langle\tilde{f}\left(\eta_{1}, \varphi_{1}\right) \tilde{f}\left(\eta_{2}, \varphi_{2}\right)\right\rangle
$$

where the averaging $\langle\ldots\rangle$ is done over all bins with $\left|\eta_{1}-\eta_{2}\right|=\Delta \eta$ and $\left|\varphi_{1}-\varphi_{2}\right|=\Delta \varphi$. Seeing structure in this correlation function would suggest the appropriate scales in rapidity and azimuth on which to bin the data to see unusual $f$ histograms. One could also do this analysis averaging over an ensemble of events. If all the events in the ensemble are selected from a single statistical distribution which is the sum of some signal distribution and some noise distribution, then the signal seen in $C(\Delta \eta, \Delta \varphi)$ would grow like the square root of the number of events in the ensemble. In this case, computing $C(\Delta \eta, \Delta \varphi)$ using an ensemble of events would be very valuable. However, if events are not selected from the same distribution, then averaging over an ensemble could wash out interesting effects. In particular, using an ensemble of events could miss seeing structures in which the interesting scales in rapidity and azimuth are different in different events, and could miss detecting effects which only occur rarely. Thus, the correlation function $C(\Delta \eta, \Delta \varphi)$ should be computed both event by event 
and for ensembles of events.

There are many ways to look for the fluctuations in the number ratio of neutral to charged pions which characterize long wavelength oscillations of the chiral condensate. We look forward to the day when the choice among analyses becomes data driven.

\subsection{Concluding Remarks}

Several different group\$1 119,112 have now done complementary simulations in which different physics has been included and different assumptions have been made. All have found that long wavelength pion oscillations can be dramatically amplified if the chiral transition is out of thermal equilibrium. This behaviour can be understood at least qualitatively in terms of a simple mechanism: as the system evolves from a disordered configuration toward a low temperature ordered configuration, nonequilibrium dynamics of the condensate can give long wavelength pion modes a negative effective mass squared, making them unstable to growth. The abrupt transition, or quench, considered in section 3.2.1 is an idealization. In a heavy ion collision, the transition presumably proceeds by a mechanism which is in some sense between the quench idealization and the thermal equilibrium idealization. There remains room for improved simulations, complementary to all that have been done so far. A $3+1$ dimensional classical simulation with boost invariant longitudinal expansion done on a large enough lattice that transverse expansion can be included as was done in $2+1$ dimensions by Asakawa et al. would be very interesting. Furthermore, if the lattice is large enough that the simulation can be run to a late enough time that the classical field amplitudes linearize, then a direct computation of the particle number would be possible. Such a simulation would complement that of Cooper et al. because although it would not include quantum fluctuations, it would not require a mean field approximation and would include transverse expansion. However, none of these simulations can come close to describing a real heavy ion collision. First of all, the linear sigma model cannot capture all the physics, although it is likely a reasonable approximation for the long wavelength modes of the chiral order parameter. Perhaps the largest uncertainty arises from the fact that we do not know what initial conditions to impose on the $\sigma$ and $\pi$ fields at the time at which a linear sigma model description of the dynamics becomes appropriate. This is crucial, because simulations have shown that expansion beginning with thermal initial conditions is not sufficient to obtain the growth of long wavelength modes. It is necessary that at the time when the linear sigma model description becomes appropriate, the long wavelength modes of the order parameter are not in equilibrium with the short wavelength modes.

The conclusions to take away from this chapter are essentially qualitative. Consider a heavy ion collision which is energetic enough that there is a central rapidity region of high energy density and low baryon number. If, as this region cools through

the chiral transition, long wavelength modes do not stay in equilibrium, then there is 
a robust mechanism which can lead to amplification of long wavelength pion oscillations. This cannot happen in thermal equilibrium. Long wavelength pion oscillations would be observed by detecting regions in rapidity-azimuth space in which the fraction of neutral pions is unusual. The pions from different long wavelength oscillations will have neutral pion fractions $f$ with an inverse square root distribution. This will of course be masked by all the other pions in the events, but the signal to noise ratio can be increased by focussing on low $p_{T}$ pions. In contrast to the equilibrium physics of chapter 2 of this article in which we were able to use the notion of universality, the nonequilibrium physics which may lead to long wavelength pion oscillations is speculative. Therefore, this subject will only become mature when it becomes experimentally driven. A dedicated experiment to look for related phenomena is underway at Fermilab. 100 Experimentalists are looking for the phenomena described in this article in current generation heavy ion experiments and will look for them in colliding beam experiments at RHIC and the LHC, where sufficient energy should be available. Detection of unusual fluctuations of $f$ in the central rapidity region of a heavy ion collision would be a dramatic and definitive signature of an out of equilibrium chiral transition. The ball is in the experimentalists' court, and we wish them well.

\section{Acknowledgements}

I first want to thank Frank Wilczek. Learning from him and working with him has been fruitful and fun. I also want to thank him for his careful reading of this article. I have had very helpful conversations with many people about different aspects of the physics I have presented. Some of these conversations happened recently, in response to an earlier draft of this article, and I am grateful for all the comments I got; others happened longer ago. My thanks to P. Bedaque, Bj, J.-P. Blaizot, W. Busza, S. Chandrasekharan, N. Christ, C. DeTar, S. Gavin, S. Hegyi, S. Hsu, Z. Huang, K. Kanaya, F. Karsch, I. Kogan, K. Kowalski, Y. Kluger, E. Laermann, R. Mawhinney, E. Mottola, B. Müller, C. Ogilvie, B. Petersson, R. Pisarski, D. Schramm, S. Selipsky, D. Spergel, M. Suzuki, C. Taylor, N. Turok, X. Wang, and B. Wyslouch.

This work was supported by the Harvard University Society of Fellows, the Milton Fund of Harvard University, and by the National Science Foundation under grant PHY-92-18167.

\section{References}

1. D. J. Gross and F. Wilczek, Phys. Rev. Lett. 30 (1973) 1343;

H. Politzer, Phys. Rev. Lett. 30 (1973) 1346.

2. J. C. Collins and M. J. Perry Phys. Rev. Lett. 34 (1975) 1353.

3. K. Rajagopal and F. Wilczek, Nucl. Phys. B399 (1993) 395.

4. K. Rajagopal and F. Wilczek, Nucl. Phys. B404 (1993) 577. 
5. D. J. Gross, R. D. Pisarski, and L. G. Yaffe, Rev. Mod. Phys. 53 (1981) 43.

6. L. McLerran, Rev. Mod. Phys. 58 (1986) 1021.

7. E. V. Shuryak, The QCD Vacuum, Hadrons, and the Superdense Matter, (World Scientific, Singapore, 1988).

8. R. Hwa, ed., Quark-Gluon Plasma, (World Scientific, Singapore, 1990).

9. B. Müller, Physics and Signatures of the Quark-Gluon Plasma., to appear in Rept. Prog. Phys., DUKE-TH-94-76 (1994) nucl-th/9410005;

B. Müller, Physics of the Quark-Gluon Plasma, Duke preprint DUKE-TH-9236, 1992.

10. Proceedings of Quark Matter '88, Nucl. Phys. A498, (1989).

11. Proceedings of Quark Matter '90, Nucl. Phys. A525, (1991).

12. Proceedings of Quark Matter '91, Nucl. Phys. A544, (1992).

13. Proceedings of Quark Matter '93, Nucl. Phys. A566, (1994).

14. B. Svetitsky and L. G. Yaffe, Nucl. Phys. B210 (1982) 423;

B. Svetitsky, Phys. Reports 132 (1986) 1.

15. L. D. Landau and E. M. Lifshitz, Statistical Physics, 2nd ed. (Pergamon, Oxford, 1969).

16. K. Wilson, Phys. Rev. D10 (1974) 2445.

Reviewed in J. Kogut, Rev. Mod. Phys. 51 (1979) 659.

17. K. Wilson, in Recent Developments in Gauge Theories, eds. G. 't Hooft et al. (Plenum, New York, 1980);

M. Creutz, L. Jacobs, and C. Rebbi, Phys. Rev. Lett. 42 (1979) 1390;

M. Creutz, Phys. Rev. Lett. 43 (1979) 553.

18. J. B. Kogut et al., Phys. Rev. Lett. 50 (1983) 393, 51 (1983) 869;

S. A. Gottlieb et al., Phys. Rev. Lett. 55 (1985) 1958;

N. H. Christ and A. E. Terrano, Phys. Rev. Lett. 56 (1986) 111.

19. S. Gottlieb et al., Phys. Rev. D35 (1987) 3972; Phys. Rev. Lett. 59 (1987) 1513; Phys. Rev. D41 (1990) 622.

20. C. Bernard et al., Phys. Rev. D45 (1992) 3854.

21. F. R. Brown et al., Phys. Rev. Lett. 65 (1990) 2491.

22. M. Fukugita, H. Mino, M. Okawa, and A. Ukawa, Phys. Rev. Lett. 65 (1990) 816; and Phys. Rev. D42 (1990) 2936.

23. S. Gottlieb et al., Phys. Rev. D47 (1993) 3619;

S. Gottlieb et al., Nucl. Phys. B Proc. Suppl. 30 (1993) 315.

24. D. Zhu, Nucl. Phys. B Proc. Suppl. 34 (1994) 286; R. Mawhinney, ibid. 30 (1993) 331.

25. Y. Iwasaki, K. Kanaya, S. Sakai, and T. Yoshié, Nucl. Phys. B Proc. Suppl. 34 (1994) 314; ibid. 30 (1992) 311; and Chiral Phase Transition in Lattice QCD with Wilson Quarks, UTHEP-300 (1995) hep-lat/9504019.

26. C. Bernard et al., QCD Thermodynamics at $N_{t}=8$ and 12, (1994) heplat/9412112. 
27. Reviews of finite temperature lattice gauge theory include

F. Karsch, p. 61 in Ref. [8]; N. Christ, p. 81 in Ref. [12]; B. Petersson, p. 237 in Ref. [11];

C. DeTar, Finite Temperature QCD: Progress and Outstanding Problems, Talk given at Lattice '94, hep-lat/9412010;

F. Karsch, Nucl. Phys. B Proc. Suppl. 34 (1994) 63;

B. Petersson, Nucl. Phys. B Proc. Suppl. 30 (1993) 66;

D. Toussaint, Nucl. Phys. B Proc. Suppl. 26 (1992) 3;

S. Gottlieb, Nucl. Phys. B Proc. Suppl. 20 (1991) 247;

C. DeTar, this volume.

28. R. Pisarski and F. Wilczek, Phys. Rev. D29 (1984) 338.

29. R. P. Feynman, Photon-Hadron Interactions, (Benjamin, Reading, MA, 1972).

30. D. H. Perkins, Introduction to High Energy Physics, (Addison-Wesley, Menlo Park, 1987).

31. J. D. Bjorken, Phys. Rev. D27 (1983) 140.

32. R. A. Salmeron, p. 1 in Quark-Gluon Plasma, B. Sinha et al., eds., (SpringerVerlag, Berlin, 1990).

33. H. Satz, p. 371 in Ref. [12].

34. A. Białas and W. Czyź, p. 271 in Ref. [8].

35. K. Geiger and B. Müller, Nucl. Phys. B369, (1992) 600;

K. Geiger, Phys. Rev. D46 (1992) 4965 and ibid. p. 4986.

36. T. S. Biró et al., Phys. Rev. C48 (1993) 1275.

37. E. Shuryak, Phys. Rev. Lett. 68 (1992) 3270;

E. Shuryak and L. Xiong, Phys. Rev. Lett. 70 (1993) 2241;

K. Geiger and J. I. Kapusta, Phys. Rev. D47 (1993) 4905;

T. Altherr and D. Seibert, Phys. Lett. B313 (1993) 149.

38. J.-P. Blaizot and J.-Y. Ollitrault, p. 393 in Ref. [8].

39. Ref. [38], p. 399.

40. F. Wilczek, Int. J. Mod. Phys. A7 (1992) 3911. This elaborates earlier work of Ref. [28].

41. G. 't Hooft, Phys. Reports 142 (1986) 357.

42. See, for example, S.-K. Ma, Modern Theory of Critical Phenomena, (Benjamin/Cummings, Reading, MA, 1976) and

D. J. Amit, Field Theory, the Renormalization Group, and Critical Phenomena, (World Scientific, Singapore, 1984).

43. M. Gell-Mann and M. Lévy, Nuovo Cimento 16 (1960) 705.

44. P. Ginsparg, Nucl. Phys. B170 (1980) 388, and references therein.

45. G. Baker, B. Nickel and D. Meiron, Phys. Rev. B17 (1978) 1365, and Compilation of 2-pt. and 4-pt. graphs for continuous spin models, unpublished, University of Guelph report (1977). 
46. K. G. Wilson and M. E. Fisher, Phys. Rev. Lett. 28 (1972) 240;

K. G. Wilson and J. Kogut, Phys. Reports 12 (1977) 77.

47. K. Kanaya and S. Kaya, Critical exponents of a three dimensional O(4) spin model, UTHEP-284 (1994) hep-lat/9409001.

48. E. Brézin, D. J. Wallace and K. G. Wilson, Phys. Rev. B7 (1973) 232.

49. E. Brézin and D. J. Wallace, Phys. Rev. B7 (1973) 1967.

50. D. J. Wallace and R. K. P. Zia, Phys. Rev. B12 (1975) 5340.

51. R. Anishetty et al., Infrared Behaviour of Systems with Goldstone Bosons, IMSc-94/52 (1995) hep-th/9502003.

52. A. Gocksch, Phys. Rev. Lett. 67 (1991) 1701.

53. T. Hatsuda and T. Kunihiro, Phys. Rev. Lett. 55 (1985) 158 and references therein.

54. M. A. Nowak, J. J. M. Verbaarschot and I. Zahed, Nucl. Phys. B325 (1989) 581

E. M. Ilgenfritz and E. V. Shuryak, Nucl. Phys. B319 (1989) 511;

E. V. Shuryak Nucl. Phys. A522 (1991) 377c.

55. E. M. Lifshitz and L. P. Pitaevskii Statistical Physics Part 2, (Pergamon, Oxford, 1980).

56. A. Kocic and J. Kogut, Can Sigma Models Describe Finite Temperature Chiral Transitions?, ILL-TH-94-19 (1994) hep-lat/9407021.

57. Articles by C. DeTar in Ref. [27].

58. K. Binder, in Phase Transitions and Critical Phenomena, Vol. 5B, ed. C. Domb and M. S. Green, (Academic Press, London, 1976), 2.

59. F. Karsch, Phys. Rev. D49 (1994) 3791.

60. F. Karsch and E. Laermann, Phys. Rev. D50 (1994) 6954.

61. For results for the energy density as a function of temperature in lattice simulations, see F. Karsch The Phase Transition to the Quark Gluon Plasma: Recent Results from Numerical Simulations, BI-TP-95-11 (1995) heplat/9503010, and references therein.

62. This point has been stressed by E. Shuryak, Comm. Nucl. Part. Phys. 21 (1994) 235.

63. A. Berera, Phys. Rev. D50 (1994) 6949; A. Berera, A Renormalization Group Approach to the Chiral Phase Transition, AZPH-TH-93-07 (1994) hep$\mathrm{ph} / 9407212$.

64. S. Chandrasekharan, Critical Behavior of the Chiral Condensate at the QCD Phase Transition, CUTP-663, (1994) hep-lat/9412070; and N. Christ and S. Chandrasekharan, private communication.

65. A. Hasenfratz and T. DeGrand, Phys. Rev. D49 (1994) 466.

66. Y. Iwasaki et al., Nucl. Phys. B Proc. Suppl. 26 (1992) 311; ibid. 30 (1993) 327; ibid. 34 (1994) 314; Phys. Rev. Lett. 69 (1992) 21; Y. Iwasaki et al. UTHEP-291 (1994) hep-lat/9412054; 
C. Bernard et al., Phys. Rev. D46 (1992) 4741; D49 (1994) 3574; T. Blum et al., Phys. Rev. D50 (1994) 3377.

67. G. Boyd et al., Nucl. Phys. B376, (1992) 199.

68. A. J. Paterson, Nucl. Phys. B190 (1981) 188.

69. H. Gausterer and S. Sanielevici, Phys. Lett. B209 (1988) 533.

70. I. Lawrie and S. Sarbach in Phase Transitions and Critical Phenomena 9 (1984) 1, ed. C. Domb and J. Lebowitz (Academic Press).

71. S. Gavin, A. Gocksch, and R. D. Pisarski, Phys. Rev. D49 (1994) 3079.

See also H. Meyer-Ortmanns and B.J. Schaefer, How sharp is the chiral crossover phenomenon for realistic meson masses?, HD-TVP-94-16 (1994) hep-ph/9409430.

72. Y. Iwasaki, K. Kanaya, S. Kaya, S. Sakai, and T. Yoshié, Nature of the finite temperature transition in QCD with strange quark, UTHEP-290 (1994) heplat/9412012;

Y. Iwasaki, Phase Diagram of QCD at Finite Temperatures with Wilson Fermions, UTHEP-293 (1994) hep-lat/9412103;

K. Kanaya, Deconfining Chiral Transition in QCD on the Lattice, UTHEP-296 (1995) hep-lat/9502020.

73. E. Witten, Phys. Rev. D30 (1984) 272;

G. Fuller, C. Alcock, and G. Mathews, Phys. Rev. D37 (1988) 1380;

J. Applegate and C. Hogan, Phys. Rev. D31 (1985) 3037.

74. D. Thomas, D. N. Schramm, K. A. Olive, G. J. Mathews, B. S. Meyer, B. D. Fields, Astrophysical J. 430 (1994) 291.

75. P. Bak and D. Mukamel, Phys. Rev. B13 (1976) 5086.

76. Z. Barak and M. B. Walker, Phys. Rev. B25 (1982) 1969.

77. J. Gasser and H. Leutwyler, Phys. Lett. B184, (1987) 83;

P. Gerber and H. Leutwyler, Nucl. Phys. B321 (1989) 387.

For a review of chiral perturbation theory at finite temperature and finite volume, see H. Leutwyler Nucl. Phys. B Proc. Suppl. 4 (1988) 248.

78. C. Contreras and M. Loewe, Int. J. Mod. Phys. A5, (1990) 2297.

79. H. Leutwyler, Nucl. Phys. B337 (1990) 108.

80. C. M. G. Lattes, Y. Fujimoto, and S. Hasegawa, Phys. Rept. 65 (1980) 151.

81. S. G. Bayburina et al., Nucl. Phys. B191 (1981) 1.

82. J. R. Ren et al., Phys. Rev. D38 (1988) 1417.

83. For recent discussions of Centauro events see: Chacaltaya and Pamir Collaboration (L. T. Baradzei et al.), Nucl. Phys. B370 (1992) 365; and articles by S. A. Slavatinsky, D. Linkai, S. Hasegawa and M. Tamada, K. Goulianos, and F. Halzen in the proceedings of the 7th Int. Symp. on Very High Energy Cosmic Ray Interactions, 1992, Ann Arbor, ed. L. Jones, (American Institute of Physics, New York, 1993).

84. R. W. Ellsworth et al., Phys. Rev. D23 (1981) 764; ibid. 771. 
85. T. H. Burnett et al., Phys. Rev. Lett. 50 (1983) 2062; Nucl. Phys. A447 (1985) 197; Phys. Rev. Lett. 57 (1986) 3249; Phys. Rev. D35 (1987) 824;

Y. Takahashi and S. Dake, Nucl. Phys. A461 (1987) 263.

86. J. J. Lord and J. Iwai, Univ. of Washington preprint (paper 515 submitted to the International Conference on High Energy Physics, Dallas, August 1992); J. Iwai (JACEE collaboration), UWSEA 92-06.

87. A. Anselm, Phys. Lett. 217B (1988) 169;

A. A. Anselm and M. G. Ryskin, Phys. Lett. B266 (1991) 482.

88. J.-P. Blaizot and A. Krzywicki, Phys. Rev. D46 (1992) 246.

89. J. D Bjorken, Int. J. Mod. Phys. A7 (1992 4189;

J. D. Bjorken, Acta Physica Polonica B23 (1992) 561.

90. K. L. Kowalski and C. C. Taylor, Disoriented Chiral Condensate: A White Paper for the Full Acceptance Detector, CWRUTH-92-6 (1992) hep$\mathrm{ph} / 9211282$.

91. J. D. Bjorken, K. L. Kowalski and C. C. Taylor, Baked Alaska, Proceedings of Les Rencontres de Physique de la Vallée D'Aoste, La Thuile, SLAC-PUB-6109 (1993); and Observing Disoriented Chiral Condensates, Proceedings of the workshop on Physics at Current Accelerators and the Supercollider, Argonne (1993) hep-ph/9309235.

92. I. V. Andreev, JETP Lett. 33 (1981) 367.

93. V. Karmanov and A. Kudrjavtsev, ITEP-88 (1983).

94. A. J. Bray, Phys. Rev. B41 (1990) 6724, and references therein;

T. J. Newman, A. J. Bray, and M. A. Moore, Phys. Rev. B42 (1990) 4514, and references therein;

J. A. N. Filipe, A. J. Bray, and S. Puri, Phase Ordering Kinetics with External Fields and Biased Initial Conditions, (1995) cond-mat/9504079.

95. N. Turok and D. N. Spergel, Phys. Rev. Lett. 66 (1991) 3093.

96. S. Gavin, A. Gocksch, and R. Pisarski, Phys. Rev. Lett. 72 (1994) 2143.

97. D. Horn and R. Silver Ann. Phys. (N.Y.) 66 (1971) 509.

98. D. Boyanovsky, H. J. de Vega, and R. Holman, Phys. Rev. D51 (1995) 734; and work presented at Journée Cosmologique, Paris (1994) hep-th/9412052.

99. K. Rajagopal, Nucl. Phys. A566 (1994) 567c.

100. J. D. Bjorken, C. C. Taylor, et al., MiniMax: A Revised Proposal for T-864, (1993);

J. D. Bjorken, MiniMax: Multiparticle Physics at the Tevatron, SLAC-PUB6430 (1994).

101. J. D. Bjorken, Disoriented Chiral Condensate, SLAC-PUB-6488, (1984); and Geometry of Multi-Hadron Production, SLAC-PUB-6707, (1994).

102. W. Heisenberg, Z. Phys. 133 (1952) 65.

103. J. C. Botke, D. J. Scalapino, and R. L. Sugar, Phys. Rev. D9 (1974) 813.

104. M. M. Enikova, V. I. Karloukovski and C. I. Velchev, Nucl. Phys. B151 
(1979) 172.

105. C. Lam and S. Lo, Phys. Rev. Lett. 52 (1984) 1184; Phys. Rev. D33 (1986) 1336; Int. J. Mod. Phys. A7 (1992) 4189.

106. S. Pratt, Phys. Lett. B301 (1993) 159;

S. Pratt and V. Zelevinsky, Phys. Rev. Lett. 72 (1994) 816.

107. C. Greiner, C. Gong and B. Müller, Phys. Lett. B316 (1993) 226.

108. R. D. Amado et al., Phys. Rev. Lett. 72 (1994) 970.

109. M. Martinis et al., Phys. Rev. D51 (1995) 2482; and hep-ph/9411329 and hep-ph/9501210.

110. S. Gavin and B. Müller, Phys. Lett. B329 (1994) 486.

111. P. F. Bedaque and A. Das, Mod. Phys. Lett. A8 (1993) 3151.

112. F. Cooper, Y. Kluger, E. Mottola, and J. P. Paz, Phys. Rev. D51 (1995) 2377 ;

F. Cooper, S. Habib, Y. Kluger, E. Mottola, J. P. Paz, P. R. Anderson, Phys. Rev. D50 (1994) 2848;

Y. Kluger, LA-UR-94-1566 (1994) hep-ph/9405279; and LA-UR-94-2754 (1994) hep-ph/9408286;

Y. Kluger et al., LBL-36904 (1995) hep-ph/9503205.

113. A. Krzywicki, Phys. Rev. D48 (1993) 5190.

114. J.-P. Blaizot and A. Krzywicki, Phys. Rev. D50 (1994) 442.

115. S. Yu. Khlebnikov, Mod. Phys. Lett. A8 (1993) 1901.

116. I. I. Kogan, Phys. Rev. D48 (1993) 3971.

117. Z. Huang and X. Wang Phys. Rev. D49 (1994) R4335.

118. A. Krzywicki, Disoriented Chiral Condensates, talk at 29th Rencontres de

Moriond, LPTHE-ORSAY-94-43 (1994) hep-ph/9405244.

119. M. Asakawa, Z. Huang, and X. Wang, LBL-35981 (1994) hep-ph/9408299.

120. Z. Huang and M. Suzuki, private communication.

121. M. Gyulassy, S. K. Kauffmann, and L. W. Wilson, Phys. Rev. C20 (1979) 2267.

122. T. D. Cohen, M. K. Banerjee, M. Nielsen, X. Jin, Phys. Lett. B333 (1994) 166.

123. I. I. Kogan, JETP Lett. 59 (1994) 307.

124. R. D. Amado and I. I. Kogan, Phys. Rev. D51 (1995) 190.

125. H. P. Yuen, Phys. Rev. A13 (1976) 2226;

B. L. Schumacher, Phys. Rep. 135 (1986) 317.

126. Z. Huang, M. Suzuki, and X. Wang, Phys. Rev. D50 (1994) 2277.

127. Z. Huang, Disoriented Chiral Condensate, LBL-36740 (1995) hepph/9501366.

128. S. Gavin, Dynamics of Chiral Symmetry Breaking in Nuclear Collisions, (1994) hep-ph/9407368;

S. Gavin, contribution to proceedings of Quark Matter '95. 
129. For example, by the WA98 experiment at CERN (B. Wyslouch, private communication); by the NA49 experiment at CERN (S. Hegyi, private communication); and by the E866 experiment at Brookhaven (C. Ogilvie, private communication).

130. This paragraph is based on preliminary studies done by B. Wyslouch and collaborators using the acceptances for the WA98 detector, discussed by him at an informal meeting at M.I.T. and in further conversations.

131. A. L. S. Angelis et al., Proposal for a Large Acceptance Hadron and Photon Spectrometer, CERN/SPSLC/91-17, May 1991;

Status Report for WA98, CERN/SPSLC/94-32, October 1994.

132. P. Bedaque, private communication. 
This figure "fig1-1.png" is available in "png" format from: http://arXiv.org/ps/hep-ph/9504310v2 
This figure "fig1-2.png" is available in "png" format from: http://arXiv.org/ps/hep-ph/9504310v2 